\title{
Detrital zircon geochronology of the Cretaceous Sindong Group, Gyeongsang Basin, Korea and its implications for paleodrainage systems
}

\author{
${ }^{1}$ Department of Energy Resources Engineering, Chosun University, Gwangju 61452, Korea \\ ${ }^{2}$ School of Earth and Environmental Sciences, Seoul National University, Seoul 08826, Korea \\ ${ }^{3}$ Department of Geological Sciences, Pusan National University, Busan 46241, Korea; *Corresponding author, E-mail: tracker@pusan.ac.kr \\ ${ }^{4}$ Department of Earth and Environmental Sciences, Hirosaki University, Aomori 036-8561, Japan
}

(Received: August 7, 2019; Revised accepted: November 27, 2019)

https://doi.org/10.18814/epiiugs/2020/020046

The Lower Cretaceous Sindong Group in southeastern Korea is a fluvio-lacustrine sedimentary package deposited in an elongated basin formed by extension in an active continental margin setting. To characterize its changes in paleodrainage system, we analyzed U-Pb ages of detrital zircons in sandstones from each stratigraphic units in northern, central, and southern parts of the Sindong Group. Detrital zircons show a wide range of ages from $106 \mathrm{Ma}$ to 3494 Ma with spatiotemporal variations in age population. Detrital zircon age spectra and paleocurrent data reveal that at least three drainage basins sourced to the Early Cretaceous river system in the Yeongnam Massif. The drainage system for the central portion of the basin was consistent with time, whereas those for the northern and southern portions shifted between the Nakdong Formation and Hasandong Formation. The strong influences resulting from the confluence of dispersal path draining Jurassic granitoids, possibly aided by faulting activity, likely caused the replacement of paleodrainage system in the northern portion. The drainage system of the southern portion was changed by extension of the drainage basin to the source terrane having similar geology to or shared the source terrane for the paleoflow system for the central portion.

\section{Introduction}

Detrital zircons offer an excellent way to track the dispersal system from source to sink. Detrital zircon geochronology has proven to be an essential technique in provenance analysis. This technique determines source rock ages, thereby further discriminating potential source areas for sediment and sediment transport systems (e.g., Rainbird et al., 1997; Gehrels and Stewart, 1998; Dickinson and Gehrels, 2003; Barth et al., 2004; Lawton et al., 2009; Raines et al., 2013). Detrital zircon geochronology has also been successfully defined sediment source areas and fluvial lineages in the modern Snake River drainage basin of the northwestern United States (Link et al., 2005). By integration of detrital zircon $\mathrm{U}-\mathrm{Pb}$ geochronology with paleocurrent analysis, Roberts et al. (2012) addressed the timing of rifting, landscape evolution, and drainage development in central-eastern Africa and showed that tectonics of continental provenance affect drainage patterns, directing, and rerouting large rivers.

The Gyeongsang Basin in southeastern Korea is a nonmarine sedimentary basin which was formed by extension in an active continental margin setting (Fig. 1). The earliest basinfill of the Gyeongsang Basin is the Sindong Group (Abtian-Albian; Lee et al., 2010), a fluvio-lacustrine sedimentary package deposited in an elongated basin (the Nakdong Trough). Petrography and paleocurrent data suggest that the western and northwestern Yeongnam Massif located to the west of the Nakdong Trough provided detritus for the Sindong Group (Chang and Kim, 1968; Koh, 1974, 1986). On the other hand, recent studies on the detrital zircon geochronology of the Sindong Group sediments (Lee et al., 2017; Lee et al., 2018a, b) revealed the Okcheon Metamorphic Belt as an additional source region of the Sindong Group.

The integration of petrographic and quartz scanning electron microscope-cathodoluminescence data led Lee et al. (2015) to conclude that the type of source rocks in the Yeongnam Massif was heterogeneous both spatially and temporally, due to different paleodrainage and paleofluvial system developed in the Nakdong Trough. They also interpreted that the temporal decrease in quartz content and increase in feldspar content resulted from tectonic events leading to the active uplift of the source areas and arc magmatism in the late stage. However, it is yet clear the relationship between different provenances and drainage patterns in the Yeongnam Massif for the Sindong Group deposition. Source rocks of the Sindong Group commonly have felsic composition that makes difficult to determine their dispersal pattern. As the formation ages of these source rocks are strikingly different, analysis of detrital zircon population of the Sindong Group is promising to track temporal and spatial changes in sediment dispersal and drainage areas of their provenance. Thus, using detrital zircon U-Pb geochronology, this study intends to document the dispersal path from 

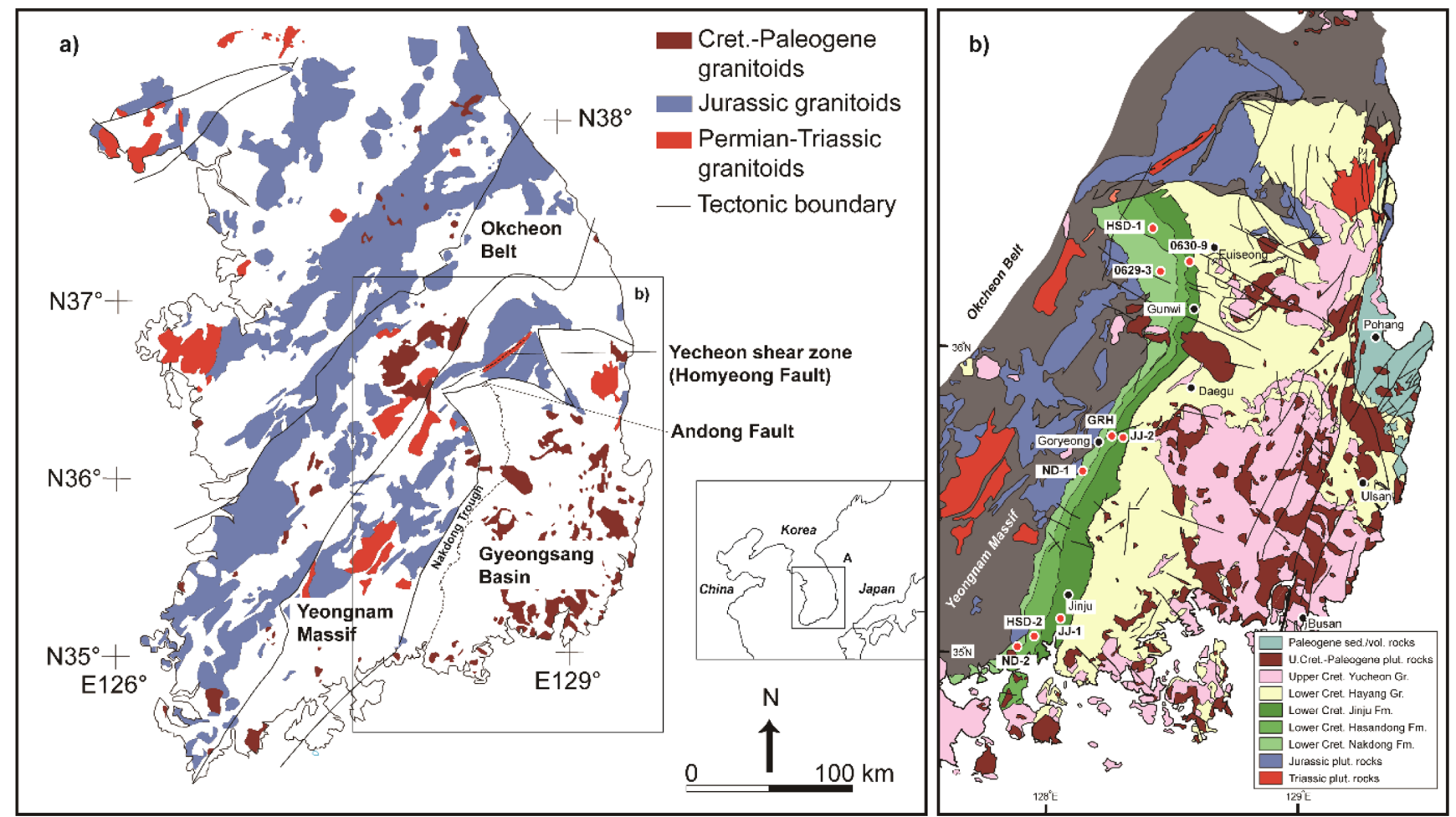

Figure 1. (a) Location of the Gyeongsang Basin and Permian-Paleogene granitoids in Korea. (b) Geologic map of the Gyeongsang Basin with sampling locations (from Lee et al., 2010).

the source to the depositional site during the Sindong Group deposition.

\section{Geological Setting}

The Gyeongsang Basin is a non-marine sedimentary basin formed in the southeastern part of Korea during Early Cretaceous time (Aptian-Albian; Lee et al., 2010) (Fig. 1). It was developed by extensional tectonics in an active continental margin caused by the subduction of the Paleo-Pacific (Izanagi) Plate beneath the East Asian continent. The Cretaceous rocks in the Gyeongsang Basin are divided into four groups based on volcanism and plutonism (Chang, 1975): they are the Sindong, Hayang, Yucheon, and the Bulguksa intrusive groups in ascending order.

The Sindong Group was deposited in an elongated basin (the Nakdong Trough; Chang, 1987) along the western margin of the Gyeongsang Basin. The Sindong Group is underlain unconformably by basement on the west side and overlain conformably by the Hayang Group on the eastern side. The Sindong Group is $2-3 \mathrm{~km}$ thick, and consists mainly of sandstone and mudrock, with minor amounts of conglomerate and marl. The Sindong Group is divided, based on lithologic features including rock color, into the Nakdong (alluvial fan to fluvial deposits), Hasandong (fluvial deposits), and Jinju (lacustrine deposits) formations with decreasing age (Chang, 1975; Choi, 1981). The Nakdong Formation is about $550 \mathrm{~m}$ thick but thickens northwards. The lowermost part of the formation consists of mid-fan conglomerates overlain by distal-fan sandstones intercalated with dark gray mudstones and black shales. The alluvial-fan deposits grade upwards into fluvial plain deposits, which are composed mainly of an alternation of channel sandstones and inter-channel dark gray mudstones and siltstones. The Hasandong Formation is approximately $1100 \mathrm{~m}$ thick and is similar to the upper part of the Nakdong Formation in that it consists of alternating channel sandstones and inter-channel fine deposits, but shows characteristic red beds containing abundant pedogenic carbonate nodules and layers (Lee, 1999). The lacustrine Jinju Formation is about $1200 \mathrm{~m}$ thick and is made up mainly of dark gray to black mudstones, deeper open lake black shales, and channel sandstones. Based on the petrographic study, most Sindong Group sediments are interpreted to have been derived from the Precambrian gneisses and Jurassic granitic rocks in the Yeongnam Massif, located to the west and northwest (Koh, 1974; Lee et al., 2010; Lee et al., 2015). However, the upper half of the Jinju Formation contains a significant amount of volcanic detritus(Choi, 1986; Noh and Park, 1990; Lee and Lim, 2008; Lee et al., 2015), suggestive of its derivation from syndepositional volcanism. Paleocurrent studies indicate that the mean direction of sediment transport was toward the east and southeast (Chang and Kim, 1968; Koh, 1986), whereas detritus in the northernmost part of the Nakdong Trough was derived mostly from the north and northwest (Rhee et al., 1988).

Deposition of the Hayang Group accompanied the basin extension to the east along with the sporadic volcanism in and around the basin (Chang, 1970, 1987). Sediments were derived from both the volcanic and plutonic-metamorphic provenances (Lee and Lee, 2000). During this interval, the Gyeongsang Basin was divided into three subbasins with different subsidence histories and sedimentary environments.

Sedimentary rocks of the Sindong Group are interpreted as the prevolcanic stage deposits, and the Hayang Group comprises volcanogenic sediments, which are intercalated with some volcanic horizons. Studies on Charophyta (Seo, 1985; Choi, 1987, 1989) and spores and 
pollen (Choi, 1985; Choi and Park, 1987) suggest that the Sindong and Hayang groups were deposited during the semi-arid climatic conditions. The volcanic and volcaniclastic rocks of the Late Cretaceous are called the Yucheon Group, and the mostly felsic plutonic rocks are collectively called the Bulguksa Granite, whose isotopic ages of granite intrusion generally ranging from ca. 120-40 Ma, and most were clustered between 85 and $70 \mathrm{Ma}$ (Shin and Jin, 1995).

Compilation of petrographic data reveals that Sindong Group sandstones are generally rich in quartz and feldspar with a mean modal composition of $\mathrm{Q}_{59} \mathrm{~F}_{31} \mathrm{R}_{10}$ (Lee et al., 2015). Quartz content decreases up sequence for all parts of the basin, whereas feldspar content has the opposite trend, with a slight increase in rock fragments. Rock fragments in the Nakdong and Hasandong sandstones are composed of metamorphic and sedimentary rocks, whereas volcanic rock fragments from contemporary arc volcanism are most common in the upper Jinju Formation sandstones, especially in the central and southern portions of the basin. Based on the spatial distribution of Lower Cretaceous detrital zircons in the Nakdong Trough, Lee et al. (2010) interpreted that location of magmatic activity in the Yeongnam Massif changed from the northern and central parts to the central and southern parts during deposition of the Sindong Group. The Gyeongsang Basin experienced maximum burial paleotemperatures of about $260^{\circ} \mathrm{C}$ (Lim et al., 2003) and underwent two cooling episodes (ca. 95$80 \mathrm{Ma}$ and after $15 \mathrm{Ma}$ ), with the first rapid exhumation facilitated by the subduction of the Izanagi-Pacific Plate ridge (Choi and Lee, 2011).

The Yeongnam Massif is a Paleoproterozoic polymetamorphic terrain bounded to the northwest by the Okcheon fold belt. The Okcheon fold belt is an intra-plate rift composed mainly of Neoproterozoic metavolcanic rocks and Paleozoic metasedimentary sequences (Cho and Kim, 2005; Cho et al., 2013). The Yeongnam Massif is made up of two Precambrian gneiss complexes: the Sobaeksan gneiss complex in the northeast and the Jirisan gneiss complex in the southwest. The Sobaeksan complex includes a broad distribution of Precambrian metasedimentary rocks, such as banded biotite gneiss, mica schist, quartzite, and calc-silicate rocks. The Jirisan complex comprises granite gneiss, porphyroblastic gneiss, augen gneiss, migmatite gneiss, and banded gneiss with minor amounts of schist and amphibolite. N-Strending Paleoproterozoic anorthosites intruded into the Jirisan complex close to the southwestern boundary of the basin. These Precambrian rocks were metamorphosed up to granulite facies (Lee et al., 1986) and were intruded by Triassic, Jurassic, and Cretaceous granites.

Triassic granitoids in the Yeongnam Massif (240-209 Ma; Cheong and Kim, 2012) are I-type plutons with metaluminous to weakly peraluminous compositions, reflecting the assimilation of crustal material. They were formed as a result of subduction-related primitive arc magmatism and are distributed mainly as small plutons in the Sobaeksan gneiss complex. Most of the Triassic granitoids were foliated during the Middle Jurassic Daebo orogeny. Jurassic granites (196-169 Ma; Cheong and Kim, 2012) form a large batholith and are generally medium- to coarse-grained porphyritic biotite granites (Jwa, 2004). They are mainly felsic I-type, peraluminous, and formed in a calcalkaline continental-arc environment (Cheong and Chang, 1996a, b; Jwa, 2004; Kim et al., 2005). The Jurassic granites are in general distributed along the long direction of the Yeongnam Massif. The Jurassic granites are thought to have been emplaced at $12-28 \mathrm{~km}$ depths (Cho and Kwon, 1994; Hong, 2001). Jwa (2004) posited that the Tri- assic and Jurassic granites can be separated into high- and low-temperature granites, respectively, based on their $\mathrm{Zr}$ contents.

Considering large exhumation of the Yeongnam Massif and the Gyeongsang Basin during the Late Cretaceous caused by the subduction of the Izanagi-Pacific Plate ridge (Choi and Lee, 2011), the present geology of the Yeongnam Massif may not represent the sourcearea characteristics during the deposition of the Sindong Group. Thus, it is one of the study purposes to compare the provenance of the Sindong Group with the current geology of the Yeongnam Massif.

\section{Methods}

A total of nine sandstone samples from the Sindong Group were collected from three different parts of the Nakdong Trough (northern, central, and southern regions; Fig. 1b). In each part of the basin, one channel sandstone sample was collected from each formation, representing the middle stratigraphic level. We mounted a total of 900 zircon grains (63-125 $\mu \mathrm{m}$ in size) (100 zircon grains from each sandstone sample) on PFA Teflon sheets. Then, the grains were polished, cleaned, and carbon-coated to determine internal structures. Cathodoluminescence $(\mathrm{CL})$ images of zircon grains were obtained using a scanning electron microscope (JEOL JSM-5400).

The analytical method for conduction detrital zircon $\mathrm{U}-\mathrm{Pb}$ age dating for the Sindong Group sandstones is the same as that followed by Lee et al. (2010). LA-ICP-MS was conducted using a Thermo Elemental PlasmaQuad 3 housed at the Earthquake Research Institute, the University of Tokyo. We used the ISOPLOT program (Ludwig, 2003 ) for data processing, and the error is quoted at $2 \sigma$. The age probability plots were constructed using the ${ }^{206} \mathrm{~Pb} /{ }^{238} \mathrm{U}$ and ${ }^{207} \mathrm{~Pb} /{ }^{206} \mathrm{~Pb}$ ages for zircons with ages younger and older than $1000 \mathrm{Ma}$, respectively. Only ages with $<15 \%$ discordance or $<10 \%$ reverse discordance were used for provenance interpretation.

\section{Results}

\section{Detrital Zircon U-Pb Ages}

Zircon grains of the Sindong Group show a wide range in color and morphology. The morphology of zircon grains ranges from euhedral prismatic crystals to irregular- and oval-shaped zircons. Detrital zircon grains showing euhedral prismatic crystal morphology are of Mesozoic in age. Among 900 zircon grains analyzed, 666 zircon grains yielded concordant or slightly discordant Archean to Cretaceous U-Pb ages. Analytical results are listed in Table 1. Among them, 46 Cretaceous $\mathrm{U}-\mathrm{Pb}$ ages were reported in Lee et al. (2010). Figure 2 shows the distribution of ages in the form of relative age probability curve, which sum probability distributions from all age analyses from a given sample into a single composite probability distribution. Most of the studied zircons show CL images of oscillatory zoning, indicating that they are of igneous origin, supported by all zircons but 18 grains have $\mathrm{Th} /$ U ratios larger than 0.1 (Table 1).

Zircon $\mathrm{U}-\mathrm{Pb}$ ages of the Sindong Group range from $106.0 \pm 1.9 \mathrm{Ma}$ to $3494 \pm 74 \mathrm{Ma}$. Most Sindong Group zircons fall into three major peak-age groups: $1870 \mathrm{Ma}(25 \%), 176-185 \mathrm{Ma}(13 \%)$, and $227 \mathrm{Ma}$ 
Table 1. Detrital zircon U-Pb ages of Sindong Group sandstones in the Gyeongsang Basin

\begin{tabular}{|c|c|c|c|c|c|c|c|c|c|}
\hline $\begin{array}{l}\text { Sample } \\
\text { Code }\end{array}$ & $\overline{\mathrm{Th} / \mathrm{U}^{2}}$ & $\begin{array}{c}{ }^{206} \mathrm{Pbc}^{(\mathrm{a})} \\
(\%)\end{array}$ & ${ }^{207} \mathrm{~Pb} /{ }^{206} \mathrm{~Pb}$ & $\begin{array}{cc}{ }^{206} \mathrm{~Pb} /{ }^{238} \mathrm{U} & \begin{array}{c}\text { Error } \\
2 \sigma\end{array} \\
\end{array}$ & $\begin{array}{c}{ }^{207} \mathrm{~Pb} /{ }^{235} \mathrm{U} \\
\begin{array}{c}\text { Error } \\
2 \sigma\end{array}\end{array}$ & $\begin{array}{c}\operatorname{Disc}^{(b)} \\
(\%)\end{array}$ & $\begin{array}{cc}{ }^{238} \mathrm{U}^{2}{ }^{206} \mathrm{~Pb} & \text { Error } \\
\text { age }(\mathrm{Ma}) & 2 \sigma\end{array}$ & $\begin{array}{cc}{ }^{235} \mathrm{U}_{-}{ }^{207} \mathrm{~Pb} & \text { Error } \\
\text { age }(\mathrm{Ma}) & 2 \sigma\end{array}$ & $\begin{array}{c}{ }^{207} \mathrm{~Pb}-{ }^{206} \mathrm{~Pb} \text { Error } \\
\text { age (Ma) } \quad 2 \sigma\end{array}$ \\
\hline \multicolumn{10}{|c|}{ Southern Nakdong Fm. $(n=80)$} \\
\hline nd2-47 & 0.73 & 0.40 & $0.0473 \pm 0.0014$ & $0.0287 \pm 0.0006$ & $0.187 \pm 0.007$ & & $182.1 \pm 3.6$ & $174.0 \pm 6.3$ & $66 \pm 2.0$ \\
\hline nd2-44 & 0.89 & n.d & $0.0539 \pm 0.0045$ & $0.0287 \pm 0.0007$ & $0.213 \pm 0.018$ & & $182.5 \pm 4.4$ & $196.3 \pm 16.9$ & $367 \pm 30.3$ \\
\hline nd2-57 & 0.75 & n.d & $0.0489 \pm 0.0018$ & $0.0290 \pm 0.0012$ & $0.195 \pm 0.011$ & & $184.1 \pm 7.4$ & $181.0 \pm 10.0$ & $142 \pm 5.3$ \\
\hline nd2-26 & 1.41 & n.d & $0.0486 \pm 0.0032$ & $0.0294 \pm 0.0014$ & $0.197 \pm 0.016$ & & $186.5 \pm 8.9$ & $182.4 \pm 14.7$ & $131 \pm 8.6$ \\
\hline nd2-63 & 0.58 & n.d & $0.0524 \pm 0.0021$ & $0.0300 \pm 0.0014$ & $0.217 \pm 0.013$ & & $190.8 \pm 9.0$ & $199.5 \pm 12.4$ & $304 \pm 12.1$ \\
\hline nd2-38 & 0.83 & n.d & $0.0484 \pm 0.0039$ & $0.0302 \pm 0.0007$ & $0.202 \pm 0.017$ & & $191.8 \pm 4.6$ & $186.5 \pm 15.8$ & $121 \pm 9.9$ \\
\hline nd2-20 & 0.49 & n.d & $0.0540 \pm 0.0024$ & $0.0303 \pm 0.0014$ & $0.225 \pm 0.014$ & & $192.3 \pm 8.9$ & $206.4 \pm 13.2$ & $371 \pm 16.4$ \\
\hline nd2-34 & 1.09 & n.d & $0.0552 \pm 0.0026$ & $0.0303 \pm 0.0008$ & $0.231 \pm 0.013$ & -0.8 & $192.5 \pm 5.2$ & $210.7 \pm 11.4$ & $420 \pm 19.8$ \\
\hline nd2-73 & 1.02 & n.d & $0.0551 \pm 0.0047$ & $0.0303 \pm 0.0014$ & $0.230 \pm 0.023$ & & $192.6 \pm 9.1$ & $210.4 \pm 20.7$ & $415 \pm 35.7$ \\
\hline nd2-79 & 0.52 & 0.71 & $0.0461 \pm 0.0026$ & $0.0307 \pm 0.0015$ & $0.195 \pm 0.015$ & & $195.1 \pm 9.7$ & $181.3 \pm 13.7$ & $6 \pm 0.3$ \\
\hline nd2-17 & 1.20 & n.d & $0.0566 \pm 0.0035$ & $0.0311 \pm 0.0015$ & $0.243 \pm 0.019$ & & $197.3 \pm 9.8$ & $220.6 \pm 17.6$ & $478 \pm 29.9$ \\
\hline nd2-87 & 1.48 & n.d & $0.0517 \pm 0.0029$ & $0.0323 \pm 0.0012$ & $0.230 \pm 0.015$ & & $204.8 \pm 7.4$ & $210.3 \pm 14.1$ & $273 \pm 15.3$ \\
\hline nd2-95 & 0.80 & n.d & $0.0529 \pm 0.0018$ & $0.0335 \pm 0.0009$ & $0.244 \pm 0.011$ & & $212.2 \pm 5.7$ & $221.9 \pm 9.7$ & $327 \pm 11.2$ \\
\hline nd2-56 & 0.80 & n.d & $0.0605 \pm 0.0026$ & $0.0343 \pm 0.0014$ & $0.286 \pm 0.017$ & -6.5 & $217.1 \pm 8.8$ & $255.1 \pm 15.2$ & $621 \pm 26.9$ \\
\hline nd2-48 & 0.82 & n.d & $0.0502 \pm 0.0017$ & $0.0362 \pm 0.0014$ & $0.250 \pm 0.013$ & & $229.0 \pm 9.2$ & $226.9 \pm 11.9$ & $205 \pm 6.9$ \\
\hline nd2-27 & 0.68 & 0.25 & $0.1164 \pm 0.0025$ & $0.2842 \pm 0.0130$ & $4.561 \pm 0.230$ & -10.9 & $1612 \pm 74$ & $1742 \pm 88$ & $1902 \pm 41$ \\
\hline nd2-75 & 0.14 & 0.14 & $0.1121 \pm 0.0022$ & $0.3026 \pm 0.0148$ & $4.679 \pm 0.246$ & -0.7 & $1704 \pm 83$ & $1763 \pm 93$ & $1835 \pm 35$ \\
\hline nd2-40 & 0.32 & 0.68 & $0.1154 \pm 0.0025$ & $0.3029 \pm 0.0059$ & $4.820 \pm 0.141$ & -6.3 & $1706 \pm 33$ & $1788 \pm 52$ & $1887 \pm 41$ \\
\hline nd2-60 & 0.13 & 0.06 & $0.1140 \pm 0.0021$ & $0.3111 \pm 0.0145$ & $4.889 \pm 0.246$ & -0.1 & $1746 \pm 82$ & $1800 \pm 91$ & $1864 \pm 35$ \\
\hline nd2-49 & 0.51 & 0.97 & $0.1209 \pm 0.0018$ & $0.3111 \pm 0.0123$ & $5.186 \pm 0.219$ & -7.2 & $1746 \pm 69$ & $1850 \pm 78$ & $1970 \pm 29$ \\
\hline nd2-74 & 0.27 & n.d & $0.1144 \pm 0.0024$ & $0.3116 \pm 0.0139$ & $4.913 \pm 0.244$ & -0.2 & $1749 \pm 78$ & $1804 \pm 89$ & $1870 \pm 40$ \\
\hline nd2-45 & 0.32 & n.d & $0.1147 \pm 0.0026$ & $0.3130 \pm 0.0062$ & $4.950 \pm 0.149$ & -2.4 & $1755 \pm 35$ & $1811 \pm 54$ & $1875 \pm 42$ \\
\hline nd2-8 & 0.19 & 0.01 & $0.1167 \pm 0.0014$ & $0.3144 \pm 0.0088$ & $5.060 \pm 0.155$ & -4.1 & $1762 \pm 49$ & $1829 \pm 56$ & $1907 \pm 23$ \\
\hline nd2-21 & 0.11 & 0.19 & $0.1141 \pm 0.0026$ & $0.3146 \pm 0.0144$ & $4.947 \pm 0.253$ & & $1763 \pm 81$ & $1810 \pm 93$ & $1865 \pm 43$ \\
\hline nd2-68 & 0.36 & 0.05 & $0.1239 \pm 0.0024$ & $0.3182 \pm 0.0142$ & $5.434 \pm 0.265$ & -6.3 & $1781 \pm 80$ & $1890 \pm 92$ & $2013 \pm 40$ \\
\hline nd2-97 & 0.05 & 0.03 & $0.1135 \pm 0.0029$ & $0.3199 \pm 0.0085$ & $5.005 \pm 0.185$ & 0.0 & $1789 \pm 48$ & $1820 \pm 67$ & $1856 \pm 48$ \\
\hline nd2-67 & 0.31 & n.d & $0.1148 \pm 0.0022$ & $0.3203 \pm 0.0150$ & $5.072 \pm 0.256$ & & $1791 \pm 84$ & $1831 \pm 92$ & $1878 \pm 36$ \\
\hline nd2-52 & 0.17 & 0.04 & $0.1169 \pm 0.0015$ & $0.3204 \pm 0.0127$ & $5.165 \pm 0.216$ & -1.2 & $1792 \pm 71$ & $1847 \pm 77$ & $1910 \pm 25$ \\
\hline nd2-50 & 0.18 & 0.15 & $0.1179 \pm 0.0015$ & $0.3207 \pm 0.0127$ & $5.214 \pm 0.217$ & -2.0 & $1793 \pm 71$ & $1855 \pm 77$ & $1925 \pm 25$ \\
\hline nd2-58 & 0.47 & n.d & $0.1146 \pm 0.0022$ & $0.3209 \pm 0.0150$ & $5.070 \pm 0.256$ & & $1794 \pm 84$ & $1831 \pm 93$ & $1874 \pm 36$ \\
\hline nd2-6 & 0.20 & 0.01 & $0.1160 \pm 0.0014$ & $0.3226 \pm 0.0091$ & $5.160 \pm 0.159$ & -1.1 & $1802 \pm 51$ & $1846 \pm 57$ & $1896 \pm 24$ \\
\hline nd2-81 & 0.04 & 0.08 & $0.1168 \pm 0.0022$ & $0.3237 \pm 0.0158$ & $5.212 \pm 0.273$ & & $1808 \pm 88$ & $1855 \pm 97$ & $1908 \pm 36$ \\
\hline nd2-72 & 0.13 & n.d & $0.1164 \pm 0.0023$ & $0.3239 \pm 0.0145$ & $5.196 \pm 0.254$ & & $1809 \pm 81$ & $1852 \pm 91$ & $1901 \pm 38$ \\
\hline nd2-59 & 0.49 & 0.35 & $0.1130 \pm 0.0024$ & $0.3259 \pm 0.0153$ & $5.077 \pm 0.261$ & & $1818 \pm 85$ & $1832 \pm 94$ & $1849 \pm 39$ \\
\hline nd2-62 & 0.18 & n.d & $0.1143 \pm 0.0022$ & $0.3262 \pm 0.0153$ & $5.140 \pm 0.261$ & & $1820 \pm 85$ & $1843 \pm 94$ & $1869 \pm 37$ \\
\hline nd2-69 & 0.18 & n.d & $0.1156 \pm 0.0024$ & $0.3286 \pm 0.0147$ & $5.237 \pm 0.258$ & & $1832 \pm 82$ & $1859 \pm 91$ & $1890 \pm 39$ \\
\hline nd2-13 & 0.26 & 0.09 & $0.1150 \pm 0.0027$ & $0.3295 \pm 0.0157$ & $5.224 \pm 0.278$ & & $1836 \pm 88$ & $1856 \pm 99$ & $1880 \pm 44$ \\
\hline nd2-98 & 0.26 & 0.42 & $0.1119 \pm 0.0030$ & $0.3321 \pm 0.0089$ & $5.125 \pm 0.195$ & & $1848 \pm 50$ & $1840 \pm 70$ & $1831 \pm 49$ \\
\hline nd2-30 & 0.40 & n.d & $0.1148 \pm 0.0027$ & $0.3373 \pm 0.0085$ & $5.340 \pm 0.183$ & & $1874 \pm 47$ & $1875 \pm 64$ & $1878 \pm 44$ \\
\hline nd2-55 & 0.25 & n.d & $0.1190 \pm 0.0016$ & $0.3381 \pm 0.0134$ & $5.548 \pm 0.233$ & & $1877 \pm 74$ & $1908 \pm 80$ & $1942 \pm 27$ \\
\hline nd2-15 & 0.11 & 0.03 & $0.1213 \pm 0.0027$ & $0.3383 \pm 0.0161$ & $5.657 \pm 0.298$ & & $1878 \pm 90$ & $1925 \pm 101$ & $1976 \pm 44$ \\
\hline nd2-10 & 0.17 & 0.04 & $0.1127 \pm 0.0026$ & $0.3393 \pm 0.0162$ & $5.274 \pm 0.280$ & & $1883 \pm 90$ & $1865 \pm 99$ & $1844 \pm 43$ \\
\hline nd2-12 & 0.47 & n.d & $0.1193 \pm 0.0027$ & $0.3397 \pm 0.0162$ & $5.586 \pm 0.295$ & & $1885 \pm 90$ & $1914 \pm 101$ & $1946 \pm 44$ \\
\hline nd2-31 & 0.46 & 0.59 & $0.1222 \pm 0.0027$ & $0.3415 \pm 0.0086$ & $5.755 \pm 0.194$ & -0.2 & $1894 \pm 48$ & $1940 \pm 65$ & $1990 \pm 45$ \\
\hline nd2-29 & 0.34 & 0.49 & $0.1118 \pm 0.0026$ & $0.3424 \pm 0.0086$ & $5.281 \pm 0.181$ & & $1898 \pm 48$ & $1866 \pm 64$ & $1830 \pm 42$ \\
\hline nd2-93 & 0.25 & n.d & $0.1165 \pm 0.0026$ & $0.3442 \pm 0.0120$ & $5.527 \pm 0.229$ & & $1907 \pm 67$ & $1905 \pm 79$ & $1903 \pm 42$ \\
\hline
\end{tabular}


Table 1. Continued

\begin{tabular}{|c|c|c|c|c|c|c|c|c|c|}
\hline $\begin{array}{l}\text { Sample } \\
\text { Code }\end{array}$ & $\overline{\mathrm{Th} / \mathrm{U}^{2}}$ & $\begin{array}{c}{ }^{206} \mathrm{Pbc}^{(\mathrm{a})} \\
(\%)\end{array}$ & ${ }^{207} \mathrm{~Pb} /{ }^{206} \mathrm{~Pb}$ & $\begin{array}{cc}{ }^{206} \mathrm{~Pb} /{ }^{238} \mathrm{U} & \begin{array}{c}\text { Error } \\
2 \sigma\end{array}\end{array}$ & $\begin{array}{c}{ }^{207} \mathrm{~Pb} /{ }^{235} \mathrm{U} \\
\text { Error } \\
2 \sigma\end{array}$ & $\begin{array}{c}\operatorname{Disc}^{(b)} \\
(\%)\end{array}$ & $\begin{array}{cc}{ }^{238} \mathrm{U}-{ }^{206} \mathrm{~Pb} & \text { Error } \\
\text { age }(\mathrm{Ma}) & 2 \sigma\end{array}$ & $\begin{array}{cc}{ }^{235} \mathrm{U}_{-}{ }^{207} \mathrm{~Pb} & \text { Error } \\
\text { age }(\mathrm{Ma}) & 2 \sigma\end{array}$ & $\begin{array}{c}{ }^{207} \mathrm{~Pb}-{ }^{206} \mathrm{~Pb} \text { Error } \\
\text { age }(\mathrm{Ma}) \quad 2 \sigma\end{array}$ \\
\hline $\mathrm{nd} 2-25$ & 0.28 & 0.35 & $0.1125 \pm 0.0025$ & $0.3456 \pm 0.0158$ & $5.362 \pm 0.273$ & & $1914 \pm 87$ & $1879 \pm 95$ & $1841 \pm 41$ \\
\hline nd2-16 & 0.35 & 0.53 & $0.1114 \pm 0.0027$ & $0.3491 \pm 0.0167$ & $5.363 \pm 0.288$ & & $1930 \pm 92$ & $1879 \pm 101$ & $1823 \pm 44$ \\
\hline nd2-85 & 0.45 & n.d & $0.1153 \pm 0.0026$ & $0.3519 \pm 0.0123$ & $5.596 \pm 0.234$ & & $1944 \pm 68$ & $1915 \pm 80$ & $1885 \pm 43$ \\
\hline nd2-33 & 0.14 & 0.04 & $0.1220 \pm 0.0026$ & $0.3522 \pm 0.0088$ & $5.926 \pm 0.196$ & & $1945 \pm 49$ & $1965 \pm 65$ & $1986 \pm 43$ \\
\hline nd2-36 & 0.84 & 0.10 & $0.1123 \pm 0.0030$ & $0.3526 \pm 0.0091$ & $5.458 \pm 0.201$ & & $1947 \pm 50$ & $1894 \pm 70$ & $1837 \pm 49$ \\
\hline nd2-78 & 0.21 & 0.17 & $0.1116 \pm 0.0023$ & $0.3536 \pm 0.0173$ & $5.439 \pm 0.288$ & & $1952 \pm 96$ & $1891 \pm 100$ & $1826 \pm 37$ \\
\hline nd2-71 & 0.25 & n.d & $0.1141 \pm 0.0023$ & $0.3571 \pm 0.0160$ & $5.616 \pm 0.275$ & & $1968 \pm 88$ & $1918 \pm 94$ & $1866 \pm 38$ \\
\hline nd2-53 & 0.21 & 0.01 & $0.1236 \pm 0.0016$ & $0.3575 \pm 0.0141$ & $6.093 \pm 0.253$ & & $1970 \pm 78$ & $1989 \pm 83$ & $2009 \pm 25$ \\
\hline nd2-7 & 1.13 & 0.04 & $0.1318 \pm 0.0019$ & $0.3630 \pm 0.0103$ & $6.595 \pm 0.208$ & -2.0 & $1996 \pm 56$ & $2059 \pm 65$ & $2122 \pm 30$ \\
\hline nd2-92 & 0.43 & n.d & $0.1217 \pm 0.0027$ & $0.3688 \pm 0.0129$ & $6.186 \pm 0.255$ & & $2024 \pm 71$ & $2002 \pm 83$ & $1981 \pm 43$ \\
\hline nd2-23 & 1.42 & 0.79 & $0.1219 \pm 0.0031$ & $0.3812 \pm 0.0175$ & $6.406 \pm 0.336$ & & $2082 \pm 96$ & $2033 \pm 107$ & $1984 \pm 50$ \\
\hline nd2-2 & 0.35 & n.d & $0.1359 \pm 0.0017$ & $0.3823 \pm 0.0108$ & $7.166 \pm 0.221$ & -0.2 & $2087 \pm 59$ & $2132 \pm 66$ & $2177 \pm 28$ \\
\hline nd2-1 & 0.35 & 0.46 & $0.1385 \pm 0.0018$ & $0.3848 \pm 0.0108$ & $7.347 \pm 0.228$ & -1.1 & $2099 \pm 59$ & $2155 \pm 67$ & $2209 \pm 29$ \\
\hline nd2-83 & 0.60 & 0.27 & $0.1501 \pm 0.0031$ & $0.3886 \pm 0.0190$ & $8.044 \pm 0.427$ & -3.8 & $2116 \pm 104$ & $2236 \pm 119$ & $2348 \pm 48$ \\
\hline nd2-22 & 0.74 & 0.23 & $0.1508 \pm 0.0033$ & $0.3907 \pm 0.0179$ & $8.121 \pm 0.412$ & -3.8 & $2126 \pm 97$ & $2245 \pm 114$ & $2355 \pm 52$ \\
\hline nd2-14 & 0.28 & n.d & $0.1549 \pm 0.0035$ & $0.3962 \pm 0.0189$ & $8.462 \pm 0.447$ & -4.3 & $2152 \pm 103$ & $2282 \pm 121$ & $2401 \pm 54$ \\
\hline nd2-41 & 0.27 & n.d & $0.1567 \pm 0.0035$ & $0.3974 \pm 0.0078$ & $8.584 \pm 0.254$ & -7.7 & $2157 \pm 43$ & $2295 \pm 68$ & $2420 \pm 53$ \\
\hline nd2-66 & 0.93 & n.d & $0.1607 \pm 0.0032$ & $0.4021 \pm 0.0188$ & $8.911 \pm 0.454$ & -6.2 & $2179 \pm 102$ & $2329 \pm 119$ & $2464 \pm 49$ \\
\hline nd2-18 & 0.73 & 0.32 & $0.1547 \pm 0.0039$ & $0.4069 \pm 0.0187$ & $8.680 \pm 0.454$ & -1.7 & $2201 \pm 101$ & $2305 \pm 121$ & $2399 \pm 60$ \\
\hline nd2-96 & 0.57 & 0.10 & $0.1604 \pm 0.0041$ & $0.4193 \pm 0.0112$ & $9.272 \pm 0.343$ & -3.5 & $2257 \pm 60$ & $2365 \pm 88$ & $2460 \pm 63$ \\
\hline nd2-89 & 0.28 & n.d & $0.1388 \pm 0.0033$ & $0.4218 \pm 0.0148$ & $8.075 \pm 0.342$ & 0.0 & $2269 \pm 80$ & $2239 \pm 95$ & $2213 \pm 52$ \\
\hline nd2-64 & 0.51 & n.d & $0.1466 \pm 0.0027$ & $0.4228 \pm 0.0198$ & $8.547 \pm 0.430$ & 0.0 & $2273 \pm 106$ & $2291 \pm 115$ & $2307 \pm 43$ \\
\hline nd2-61 & 1.14 & n.d & $0.1612 \pm 0.0034$ & $0.4242 \pm 0.0199$ & $9.426 \pm 0.484$ & -1.3 & $2279 \pm 107$ & $2380 \pm 122$ & $2468 \pm 52$ \\
\hline nd2-43 & 1.41 & n.d & $0.1612 \pm 0.0039$ & $0.4270 \pm 0.0087$ & $9.489 \pm 0.299$ & -3.1 & $2292 \pm 47$ & $2386 \pm 75$ & $2469 \pm 60$ \\
\hline nd2-28 & 0.62 & n.d & $0.1498 \pm 0.0035$ & $0.4367 \pm 0.0111$ & $9.022 \pm 0.312$ & & $2336 \pm 59$ & $2340 \pm 81$ & $2345 \pm 55$ \\
\hline nd2-100 & 0.21 & n.d & $0.1576 \pm 0.0041$ & $0.4371 \pm 0.0117$ & $9.498 \pm 0.352$ & & $2338 \pm 62$ & $2387 \pm 89$ & $2430 \pm 63$ \\
\hline nd2-84 & 0.97 & n.d & $0.1659 \pm 0.0039$ & $0.4436 \pm 0.0219$ & $10.148 \pm 0.556$ & & $2367 \pm 117$ & $2448 \pm 134$ & $2517 \pm 60$ \\
\hline nd2-91 & 0.37 & 0.04 & $0.1501 \pm 0.0033$ & $0.4471 \pm 0.0156$ & $9.249 \pm 0.380$ & & $2382 \pm 83$ & $2363 \pm 97$ & $2347 \pm 51$ \\
\hline nd2-4 & 0.96 & 0.13 & $0.1608 \pm 0.0021$ & $0.4550 \pm 0.0128$ & $10.085 \pm 0.314$ & & $2417 \pm 68$ & $2442 \pm 76$ & $2464 \pm 32$ \\
\hline nd2-32 & 1.33 & 0.44 & $0.1601 \pm 0.0038$ & $0.4573 \pm 0.0117$ & $10.097 \pm 0.352$ & & $2428 \pm 62$ & $2444 \pm 85$ & $2457 \pm 58$ \\
\hline nd2-76 & 0.95 & n.d & $0.1676 \pm 0.0032$ & $0.4618 \pm 0.0226$ & $10.671 \pm 0.562$ & & $2448 \pm 120$ & $2495 \pm 131$ & $2534 \pm 49$ \\
\hline nd2-86 & 0.11 & n.d & $0.1670 \pm 0.0036$ & $0.4753 \pm 0.0166$ & $10.943 \pm 0.449$ & & $2507 \pm 88$ & $2518 \pm 103$ & $2528 \pm 55$ \\
\hline nd2-90 & 0.62 & 0.13 & $0.1645 \pm 0.0036$ & $0.5062 \pm 0.0177$ & $11.483 \pm 0.475$ & & $2640 \pm 92$ & $2563 \pm 106$ & $2503 \pm 55$ \\
\hline nd2-46 & 1.05 & n.d & $0.3045 \pm 0.0065$ & $0.6116 \pm 0.0121$ & $25.677 \pm 0.747$ & -9.2 & $3077 \pm 61$ & $3334 \pm 97$ & $3494 \pm 75$ \\
\hline \multicolumn{10}{|c|}{ Central Nakdong Fm. $(n=58)$} \\
\hline nd1-54 & 1.47 & 0.10 & $0.0476 \pm 0.0020$ & $0.0185 \pm 0.0005$ & $0.121 \pm 0.006$ & & $118.0 \pm 3.0$ & $116.2 \pm 5.7$ & $80 \pm 3.4$ \\
\hline nd1-46 & 0.41 & 0.02 & $0.0539 \pm 0.0021$ & $0.0191 \pm 0.0005$ & $0.142 \pm 0.007$ & -3.0 & $122.2 \pm 2.9$ & $135.0 \pm 6.2$ & $367 \pm 14.5$ \\
\hline nd1-8 & 0.80 & n.d & $0.0477 \pm 0.0026$ & $0.0216 \pm 0.0011$ & $0.142 \pm 0.011$ & & $137.9 \pm 7.1$ & $134.9 \pm 10.2$ & $83 \pm 4.6$ \\
\hline nd1-63 & 0.88 & n.d & $0.0509 \pm 0.0019$ & $0.0264 \pm 0.0007$ & $0.185 \pm 0.008$ & & $167.8 \pm 4.3$ & $172.6 \pm 7.7$ & $239 \pm 8.7$ \\
\hline nd1-40 & 0.57 & n.d & $0.0528 \pm 0.0016$ & $0.0265 \pm 0.0006$ & $0.193 \pm 0.007$ & & $168.8 \pm 3.9$ & $179.2 \pm 6.8$ & $320 \pm 9.6$ \\
\hline nd1-52 & 0.45 & n.d & $0.0478 \pm 0.0017$ & $0.0267 \pm 0.0007$ & $0.176 \pm 0.008$ & & $170.1 \pm 4.3$ & $164.8 \pm 7.2$ & $90 \pm 3.2$ \\
\hline nd1-25 & 0.49 & n.d & $0.0505 \pm 0.0026$ & $0.0268 \pm 0.0009$ & $0.186 \pm 0.012$ & & $170.3 \pm 5.8$ & $173.5 \pm 10.8$ & $218 \pm 11.4$ \\
\hline nd1-26 & 0.92 & n.d & $0.0508 \pm 0.0017$ & $0.0278 \pm 0.0009$ & $0.195 \pm 0.009$ & & $177.1 \pm 5.9$ & $180.8 \pm 8.6$ & $231 \pm 7.9$ \\
\hline nd1-32 & 0.97 & n.d & $0.0515 \pm 0.0015$ & $0.0283 \pm 0.0006$ & $0.201 \pm 0.007$ & & $179.7 \pm 3.8$ & $185.6 \pm 6.8$ & $262 \pm 7.8$ \\
\hline nd1-10 & 0.90 & n.d & $0.0486 \pm 0.0018$ & $0.0286 \pm 0.0014$ & $0.192 \pm 0.012$ & & $182.0 \pm 9.2$ & $178.3 \pm 11.1$ & $130 \pm 4.7$ \\
\hline nd1-29 & 0.68 & n.d & $0.0504 \pm 0.0013$ & $0.0294 \pm 0.0006$ & $0.204 \pm 0.007$ & & $186.9 \pm 4.0$ & $188.7 \pm 6.4$ & $212 \pm 5.6$ \\
\hline nd1-43 & 1.26 & n.d & $0.0520 \pm 0.0017$ & $0.0295 \pm 0.0007$ & $0.211 \pm 0.009$ & & $187.2 \pm 4.4$ & $194.6 \pm 7.9$ & $287 \pm 9.5$ \\
\hline
\end{tabular}


Table 1. Continued

\begin{tabular}{|c|c|c|c|c|c|c|c|c|c|}
\hline $\begin{array}{l}\text { Sample } \\
\text { Code }\end{array}$ & $\mathrm{Th} / \mathrm{U}$ & $\begin{array}{c}{ }^{206} \mathrm{Pbc}^{(\mathrm{a})} \\
(\%)\end{array}$ & ${ }^{207} \mathrm{~Pb} /{ }^{206} \mathrm{~Pb}$ & $\begin{array}{cc}{ }^{206} \mathrm{~Pb} /{ }^{238} \mathrm{U} & \begin{array}{c}\text { Error } \\
2 \sigma\end{array}\end{array}$ & $\begin{array}{c}{ }^{207} \mathrm{~Pb} /{ }^{235} \mathrm{U} \\
\text { Error } \\
2 \sigma\end{array}$ & $\begin{array}{c}\operatorname{Disc}^{(b)} \\
(\%)\end{array}$ & $\begin{array}{cc}{ }^{238} \mathrm{U}-{ }^{206} \mathrm{~Pb} & \text { Error } \\
\text { age }(\mathrm{Ma}) & 2 \sigma\end{array}$ & $\begin{array}{c}{ }^{235} \mathrm{U}_{-}{ }^{207} \mathrm{~Pb} \\
\text { age }(\mathrm{Ma})\end{array}$ & $\begin{array}{c}{ }^{207} \mathrm{~Pb}-{ }^{206} \mathrm{~Pb} \text { Error } \\
\text { age (Ma) } \quad 2 \sigma\end{array}$ \\
\hline nd1-15 & 0.88 & n.d & $0.0565 \pm 0.0029$ & $0.0300 \pm 0.0015$ & $0.234 \pm 0.017$ & & $190.8 \pm 9.8$ & $213.4 \pm 15.3$ & $471 \pm 23.8$ \\
\hline nd1-48 & 0.89 & n.d & $0.0595 \pm 0.0033$ & $0.0305 \pm 0.0008$ & $0.250 \pm 0.016$ & -7.0 & $193.4 \pm 5.3$ & $226.4 \pm 14.1$ & $586 \pm 32.8$ \\
\hline nd1-31 & 1.33 & 0.54 & $0.0612 \pm 0.0027$ & $0.0313 \pm 0.0007$ & $0.264 \pm 0.013$ & -11.6 & $198.5 \pm 4.5$ & $237.8 \pm 11.7$ & $647 \pm 28.2$ \\
\hline nd1-4 & 0.22 & n.d & $0.0544 \pm 0.0038$ & $0.0331 \pm 0.0015$ & $0.248 \pm 0.021$ & & $210.0 \pm 9.8$ & $225.2 \pm 18.9$ & $388 \pm 27.0$ \\
\hline nd1-42 & 0.09 & n.d & $0.0502 \pm 0.0015$ & $0.0354 \pm 0.0008$ & $0.245 \pm 0.009$ & & $224.4 \pm 5.2$ & $222.8 \pm 8.3$ & $207 \pm 6.1$ \\
\hline nd1-45 & 0.07 & n.d & $0.0515 \pm 0.0021$ & $0.0358 \pm 0.0009$ & $0.254 \pm 0.012$ & & $226.9 \pm 5.4$ & $230.2 \pm 10.7$ & $264 \pm 10.6$ \\
\hline nd1-16 & 0.88 & 0.52 & $0.0477 \pm 0.0015$ & $0.0361 \pm 0.0018$ & $0.238 \pm 0.014$ & & $228.7 \pm 11.5$ & $216.4 \pm 12.7$ & $84 \pm 2.6$ \\
\hline nd1-33 & 0.48 & n.d & $0.0542 \pm 0.0015$ & $0.0416 \pm 0.0009$ & $0.311 \pm 0.011$ & & $262.4 \pm 5.6$ & $274.8 \pm 9.7$ & $382 \pm 10.7$ \\
\hline nd1-30 & 0.74 & n.d & $0.0506 \pm 0.0020$ & $0.0449 \pm 0.0010$ & $0.313 \pm 0.014$ & & $283.1 \pm 6.3$ & $276.8 \pm 12.7$ & $224 \pm 9.0$ \\
\hline nd1-14 & 0.66 & n.d & $0.0628 \pm 0.0019$ & $0.0744 \pm 0.0037$ & $0.644 \pm 0.038$ & & $462.3 \pm 23.3$ & $504.7 \pm 29.5$ & $702 \pm 20.8$ \\
\hline nd1-21 & 0.49 & 0.39 & $0.0614 \pm 0.0015$ & $0.0942 \pm 0.0031$ & $0.797 \pm 0.033$ & & $580.5 \pm 19.1$ & $595.2 \pm 24.7$ & $652 \pm 16.4$ \\
\hline nd1-67 & 0.29 & n.d & $0.0637 \pm 0.0012$ & $0.0985 \pm 0.0024$ & $0.864 \pm 0.027$ & & $605.3 \pm 15.0$ & $632.4 \pm 19.8$ & $731 \pm 13.9$ \\
\hline nd1-24 & 1.47 & 10.92 & $0.0591 \pm 0.0016$ & $0.1096 \pm 0.0036$ & $0.894 \pm 0.038$ & & $670.7 \pm 22.3$ & $648.5 \pm 27.8$ & $573 \pm 15.5$ \\
\hline nd $1-28$ & 1.46 & 0.62 & $0.0598 \pm 0.0016$ & $0.1170 \pm 0.0025$ & $0.964 \pm 0.033$ & & $713.4 \pm 15.2$ & $685.4 \pm 23.5$ & $595 \pm 16.0$ \\
\hline nd1-9 & 0.95 & n.d & $0.0671 \pm 0.0018$ & $0.1293 \pm 0.0065$ & $1.196 \pm 0.069$ & & $784.1 \pm 39.5$ & $798.8 \pm 45.8$ & $841 \pm 23.1$ \\
\hline nd1-2 & 0.73 & n.d & $0.0681 \pm 0.0017$ & $0.1302 \pm 0.0059$ & $1.223 \pm 0.063$ & & $788.9 \pm 35.6$ & $811.1 \pm 41.9$ & $873 \pm 21.9$ \\
\hline nd1-12 & 0.40 & n.d & $0.0673 \pm 0.0025$ & $0.1335 \pm 0.0068$ & $1.240 \pm 0.078$ & & $807.8 \pm 41.0$ & $818.7 \pm 51.7$ & $849 \pm 31.8$ \\
\hline nd1-6 & 0.77 & n.d & $0.0668 \pm 0.0016$ & $0.1360 \pm 0.0061$ & $1.253 \pm 0.064$ & & $822.2 \pm 37.1$ & $824.7 \pm 42.3$ & $832 \pm 20.3$ \\
\hline nd1-17 & 1.13 & n.d & $0.0680 \pm 0.0025$ & $0.1403 \pm 0.0071$ & $1.315 \pm 0.083$ & & $846.3 \pm 43.0$ & $852.4 \pm 53.7$ & $869 \pm 32.3$ \\
\hline nd1-57 & 1.11 & 0.43 & $0.0850 \pm 0.0015$ & $0.2243 \pm 0.0055$ & $2.627 \pm 0.080$ & & $1304 \pm 32$ & $1308 \pm 40$ & $1315 \pm 23$ \\
\hline nd1-37 & 0.57 & 0.33 & $0.1147 \pm 0.0025$ & $0.2856 \pm 0.0060$ & $4.516 \pm 0.138$ & -11.2 & $1619 \pm 34$ & $1734 \pm 53$ & $1876 \pm 41$ \\
\hline nd1-23 & 0.20 & 0.01 & $0.1141 \pm 0.0027$ & $0.2867 \pm 0.0094$ & $4.512 \pm 0.182$ & -8.9 & $1625 \pm 53$ & $1733 \pm 70$ & $1867 \pm 43$ \\
\hline nd1-58 & 0.54 & n.d & $0.1169 \pm 0.0018$ & $0.2948 \pm 0.0073$ & $4.749 \pm 0.138$ & -10.4 & $1665 \pm 41$ & $1776 \pm 52$ & $1909 \pm 29$ \\
\hline nd1-51 & 0.15 & 0.17 & $0.1139 \pm 0.0017$ & $0.2972 \pm 0.0073$ & $4.669 \pm 0.134$ & -6.9 & $1678 \pm 41$ & $1762 \pm 51$ & $1863 \pm 28$ \\
\hline nd1-50 & 1.23 & 0.15 & $0.1146 \pm 0.0017$ & $0.2988 \pm 0.0073$ & $4.722 \pm 0.135$ & -7.1 & $1685 \pm 41$ & $1771 \pm 51$ & $1874 \pm 28$ \\
\hline nd1-66 & 0.63 & n.d & $0.1134 \pm 0.0019$ & $0.2999 \pm 0.0074$ & $4.690 \pm 0.140$ & -5.4 & $1691 \pm 42$ & $1765 \pm 53$ & $1855 \pm 31$ \\
\hline nd1-7 & 0.26 & n.d & $0.1179 \pm 0.0024$ & $0.3001 \pm 0.0135$ & $4.880 \pm 0.240$ & -7.1 & $1692 \pm 76$ & $1799 \pm 89$ & $1926 \pm 38$ \\
\hline nd1-18 & 0.12 & 0.04 & $0.1151 \pm 0.0026$ & $0.3041 \pm 0.0100$ & $4.826 \pm 0.193$ & -4.1 & $1712 \pm 56$ & $1789 \pm 72$ & $1882 \pm 43$ \\
\hline nd1-62 & 0.21 & n.d & $0.1170 \pm 0.0018$ & $0.3052 \pm 0.0075$ & $4.925 \pm 0.143$ & -7.2 & $1717 \pm 42$ & $1806 \pm 53$ & $1912 \pm 29$ \\
\hline nd1-1 & 0.51 & n.d & $0.1155 \pm 0.0025$ & $0.3069 \pm 0.0138$ & $4.887 \pm 0.244$ & -2.5 & $1726 \pm 78$ & $1800 \pm 90$ & $1888 \pm 41$ \\
\hline nd1-59 & 0.23 & n.d & $0.1157 \pm 0.0017$ & $0.3085 \pm 0.0076$ & $4.923 \pm 0.142$ & -5.1 & $1733 \pm 43$ & $1806 \pm 52$ & $1892 \pm 28$ \\
\hline nd1-13 & 0.24 & n.d & $0.1172 \pm 0.0030$ & $0.3099 \pm 0.0156$ & $5.008 \pm 0.283$ & -2.2 & $1740 \pm 88$ & $1821 \pm 103$ & $1915 \pm 49$ \\
\hline nd $1-55$ & 0.18 & 0.13 & $0.1138 \pm 0.0017$ & $0.3102 \pm 0.0076$ & $4.865 \pm 0.139$ & -2.8 & $1742 \pm 43$ & $1796 \pm 51$ & $1861 \pm 27$ \\
\hline nd $1-44$ & 0.18 & n.d & $0.1144 \pm 0.0024$ & $0.3107 \pm 0.0071$ & $4.900 \pm 0.151$ & -2.7 & $1744 \pm 40$ & $1802 \pm 55$ & $1870 \pm 39$ \\
\hline nd1-56 & 0.29 & n.d & $0.1155 \pm 0.0017$ & $0.3184 \pm 0.0078$ & $5.069 \pm 0.144$ & -2.0 & $1782 \pm 43$ & $1831 \pm 52$ & $1888 \pm 27$ \\
\hline nd1-36 & 1.11 & n.d & $0.1151 \pm 0.0025$ & $0.3232 \pm 0.0068$ & $5.127 \pm 0.154$ & & $1805 \pm 38$ & $1840 \pm 55$ & $1881 \pm 40$ \\
\hline nd1-60 & 0.39 & 0.03 & $0.1162 \pm 0.0018$ & $0.3238 \pm 0.0080$ & $5.190 \pm 0.151$ & -0.9 & $1808 \pm 45$ & $1851 \pm 54$ & $1899 \pm 29$ \\
\hline nd1-27 & 0.43 & 0.01 & $0.1275 \pm 0.0030$ & $0.3355 \pm 0.0110$ & $5.898 \pm 0.238$ & -4.8 & $1865 \pm 61$ & $1961 \pm 79$ & $2064 \pm 48$ \\
\hline nd1-20 & 0.85 & 0.13 & $0.1232 \pm 0.0030$ & $0.3538 \pm 0.0117$ & $6.011 \pm 0.246$ & & $1953 \pm 64$ & $1977 \pm 81$ & $2004 \pm 49$ \\
\hline nd1-47 & 0.38 & n.d & $0.1221 \pm 0.0025$ & $0.3724 \pm 0.0085$ & $6.271 \pm 0.193$ & & $2041 \pm 46$ & $2014 \pm 62$ & $1988 \pm 41$ \\
\hline nd1-3 & 0.70 & n.d & $0.1459 \pm 0.0029$ & $0.4039 \pm 0.0182$ & $8.128 \pm 0.399$ & & $2187 \pm 98$ & $2245 \pm 110$ & $2299 \pm 45$ \\
\hline nd $1-65$ & 0.38 & 0.05 & $0.1480 \pm 0.0023$ & $0.4253 \pm 0.0105$ & $8.677 \pm 0.252$ & & $2284 \pm 56$ & $2305 \pm 67$ & $2323 \pm 35$ \\
\hline nd1-39 & 0.47 & 0.08 & $0.1814 \pm 0.0037$ & $0.4298 \pm 0.0098$ & $10.752 \pm 0.329$ & -11.0 & $2305 \pm 52$ & $2502 \pm 76$ & $2666 \pm 54$ \\
\hline nd1-11 & 0.95 & n.d & $0.1754 \pm 0.0046$ & $0.4935 \pm 0.0249$ & $11.933 \pm 0.677$ & & $2586 \pm 130$ & $2599 \pm 147$ & $2610 \pm 68$ \\
\hline nd1-61 & 0.43 & 7.44 & $0.1191 \pm 0.0018$ & $0.5391 \pm 0.0133$ & $8.852 \pm 0.256$ & & $2780 \pm 69$ & $2323 \pm 67$ & $1943 \pm 29$ \\
\hline nd1-5 & 0.52 & n.d & $0.2703 \pm 0.0053$ & $0.5768 \pm 0.0261$ & $21.496 \pm 1.060$ & -5.9 & $2936 \pm 133$ & $3161 \pm 156$ & $3308 \pm 65$ \\
\hline
\end{tabular}


Table 1. Continued

\begin{tabular}{|c|c|c|c|c|c|c|c|c|c|}
\hline $\begin{array}{l}\text { Sample } \\
\text { Code }\end{array}$ & $\mathrm{Th} / \mathrm{U}$ & $\begin{array}{c}{ }^{206} \mathrm{Pbc}^{(\mathrm{a})} \\
(\%)\end{array}$ & ${ }^{207} \mathrm{~Pb} /{ }^{206} \mathrm{~Pb}$ & $\begin{array}{cc}{ }^{206} \mathrm{~Pb} /{ }^{238} \mathrm{U} & \begin{array}{c}\text { Error } \\
2 \sigma\end{array}\end{array}$ & $\begin{array}{c}{ }^{207} \mathrm{~Pb} /{ }^{235} \mathrm{U} \begin{array}{c}\text { Error } \\
2 \sigma\end{array} \\
\end{array}$ & $\begin{array}{c}\operatorname{Disc}^{(b)} \\
(\%)\end{array}$ & $\begin{array}{cc}{ }^{238} \mathrm{U}-{ }^{206} \mathrm{~Pb} & \text { Error } \\
\text { age }(\mathrm{Ma}) & 2 \sigma\end{array}$ & $\begin{array}{cc}{ }^{235} \mathrm{U}^{2}{ }^{207} \mathrm{~Pb} & \text { Error } \\
\text { age }(\mathrm{Ma}) & 2 \sigma\end{array}$ & $\begin{array}{l}{ }^{207} \mathrm{~Pb}-{ }^{206} \mathrm{~Pb} \text { Error } \\
\text { age }(\mathrm{Ma}) \quad 2 \sigma\end{array}$ \\
\hline \multicolumn{10}{|c|}{ Northern Nakdong Fm. $(n=89)$} \\
\hline $0629-3-55$ & 0.81 & n.d & $0.0527 \pm 0.0018$ & $0.0191 \pm 0.0005$ & $0.139 \pm 0.006$ & -0.5 & $122.0 \pm 3.5$ & $132.0 \pm 5.9$ & $317 \pm 11.0$ \\
\hline $0629-3-25$ & 1.25 & n.d & $0.0483 \pm 0.0019$ & $0.0240 \pm 0.0005$ & $0.160 \pm 0.007$ & & $152.8 \pm 3.2$ & $150.6 \pm 6.7$ & $115 \pm 4.5$ \\
\hline $0629-3-100$ & 0.74 & n.d & $0.0536 \pm 0.0041$ & $0.0247 \pm 0.0006$ & $0.182 \pm 0.015$ & & $157.1 \pm 3.6$ & $170.0 \pm 13.6$ & $355 \pm 27.2$ \\
\hline $0629-3-54$ & 1.05 & n.d & $0.0559 \pm 0.0029$ & $0.0247 \pm 0.0007$ & $0.190 \pm 0.011$ & -2.8 & $157.1 \pm 4.7$ & $176.8 \pm 10.7$ & $450 \pm 23.6$ \\
\hline $0629-3-21$ & 0.89 & n.d & $0.0486 \pm 0.0031$ & $0.0247 \pm 0.0006$ & $0.166 \pm 0.011$ & & $157.3 \pm 3.5$ & $155.5 \pm 10.4$ & $129 \pm 8.1$ \\
\hline $0629-3-35$ & 1.04 & n.d & $0.0504 \pm 0.0030$ & $0.0253 \pm 0.0005$ & $0.176 \pm 0.011$ & & $161.2 \pm 3.5$ & $164.7 \pm 10.5$ & $216 \pm 13.0$ \\
\hline $0629-3-62$ & 0.96 & n.d & $0.0513 \pm 0.0034$ & $0.0253 \pm 0.0011$ & $0.179 \pm 0.014$ & & $161.3 \pm 7.1$ & $167.3 \pm 13.3$ & $254 \pm 16.7$ \\
\hline $0629-3-6$ & 0.67 & n.d & $0.0466 \pm 0.0031$ & $0.0255 \pm 0.0012$ & $0.164 \pm 0.013$ & & $162.4 \pm 7.8$ & $154.0 \pm 12.5$ & $28 \pm 1.8$ \\
\hline $0629-3-36$ & 0.69 & n.d & $0.0474 \pm 0.0038$ & $0.0256 \pm 0.0006$ & $0.167 \pm 0.014$ & & $163.2 \pm 3.7$ & $157.2 \pm 13.3$ & $68 \pm 5.5$ \\
\hline $0629-3-98$ & 1.01 & n.d & $0.0490 \pm 0.0022$ & $0.0259 \pm 0.0005$ & $0.175 \pm 0.009$ & & $164.6 \pm 3.5$ & $163.4 \pm 8.0$ & $147 \pm 6.5$ \\
\hline $0629-3-45$ & 0.90 & n.d & $0.0500 \pm 0.0020$ & $0.0262 \pm 0.0005$ & $0.180 \pm 0.008$ & & $166.4 \pm 3.1$ & $168.3 \pm 7.3$ & $195 \pm 7.6$ \\
\hline $0629-3-2$ & 0.66 & n.d & $0.0536 \pm 0.0037$ & $0.0268 \pm 0.0013$ & $0.198 \pm 0.017$ & & $170.6 \pm 8.3$ & $183.8 \pm 15.4$ & $357 \pm 24.4$ \\
\hline $0629-3-82$ & 2.07 & n.d & $0.0553 \pm 0.0028$ & $0.0269 \pm 0.0009$ & $0.205 \pm 0.012$ & -0.9 & $171.4 \pm 5.5$ & $189.7 \pm 11.3$ & $425 \pm 21.5$ \\
\hline $0629-3-65$ & 0.32 & n.d & $0.0530 \pm 0.0027$ & $0.0270 \pm 0.0012$ & $0.198 \pm 0.013$ & & $172.1 \pm 7.5$ & $183.1 \pm 12.3$ & $329 \pm 16.8$ \\
\hline $0629-3-77$ & 0.30 & n.d & $0.0486 \pm 0.0014$ & $0.0271 \pm 0.0007$ & $0.182 \pm 0.007$ & & $172.5 \pm 4.5$ & $169.7 \pm 6.6$ & $131 \pm 3.8$ \\
\hline $0629-3-51$ & 0.29 & n.d & $0.0487 \pm 0.0017$ & $0.0272 \pm 0.0008$ & $0.182 \pm 0.008$ & & $172.8 \pm 4.9$ & $170.1 \pm 7.7$ & $134 \pm 4.7$ \\
\hline $0629-3-61$ & 1.36 & n.d & $0.0479 \pm 0.0029$ & $0.0276 \pm 0.0012$ & $0.182 \pm 0.014$ & & $175.7 \pm 7.7$ & $170.1 \pm 12.8$ & $93 \pm 5.7$ \\
\hline $0629-3-89$ & 0.25 & n.d & $0.0473 \pm 0.0016$ & $0.0282 \pm 0.0009$ & $0.184 \pm 0.008$ & & $179.0 \pm 5.6$ & $171.1 \pm 7.8$ & $65 \pm 2.2$ \\
\hline $0629-3-29$ & 1.29 & n.d & $0.0438 \pm 0.0023$ & $0.0288 \pm 0.0006$ & $0.174 \pm 0.010$ & & $182.8 \pm 3.9$ & $162.8 \pm 9.2$ & \\
\hline $0629-3-75$ & 0.33 & n.d & $0.0482 \pm 0.0015$ & $0.0309 \pm 0.0008$ & $0.206 \pm 0.008$ & & $196.5 \pm 5.1$ & $189.9 \pm 7.8$ & $110 \pm 3.4$ \\
\hline $0629-3-10$ & 1.61 & n.d & $0.0508 \pm 0.0017$ & $0.0313 \pm 0.0015$ & $0.219 \pm 0.012$ & & $198.4 \pm 9.3$ & $200.9 \pm 11.5$ & $231 \pm 7.5$ \\
\hline 0629-3-19 & 0.67 & n.d & $0.0534 \pm 0.0018$ & $0.0331 \pm 0.0013$ & $0.244 \pm 0.012$ & & $210.0 \pm 8.0$ & $221.4 \pm 11.3$ & $344 \pm 11.7$ \\
\hline $0629-3-13$ & 0.06 & 0.28 & $0.0505 \pm 0.0017$ & $0.0345 \pm 0.0013$ & $0.240 \pm 0.012$ & & $218.4 \pm 8.3$ & $218.5 \pm 11.1$ & $220 \pm 7.4$ \\
\hline $0629-3-57$ & 1.30 & n.d & $0.0518 \pm 0.0015$ & $0.0345 \pm 0.0010$ & $0.247 \pm 0.010$ & & $218.8 \pm 6.2$ & $223.8 \pm 9.1$ & $278 \pm 8.2$ \\
\hline $0629-3-66$ & 0.49 & n.d & $0.0496 \pm 0.0039$ & $0.0354 \pm 0.0016$ & $0.242 \pm 0.022$ & & $224.5 \pm 10.1$ & $220.3 \pm 19.9$ & $177 \pm 13.9$ \\
\hline $0629-3-22$ & 0.07 & n.d & $0.0517 \pm 0.0022$ & $0.0355 \pm 0.0007$ & $0.253 \pm 0.012$ & & $225.0 \pm 4.7$ & $229.3 \pm 10.8$ & $274 \pm 11.5$ \\
\hline $0629-3-37$ & 2.63 & n.d & $0.0514 \pm 0.0033$ & $0.0360 \pm 0.0008$ & $0.255 \pm 0.017$ & & $228.0 \pm 5.0$ & $230.6 \pm 15.6$ & $258 \pm 16.5$ \\
\hline $0629-3-47$ & 1.03 & n.d & $0.0507 \pm 0.0016$ & $0.0363 \pm 0.0007$ & $0.254 \pm 0.009$ & & $229.8 \pm 4.1$ & $229.6 \pm 8.5$ & $229 \pm 7.4$ \\
\hline $0629-3-71$ & 0.02 & n.d & $0.0497 \pm 0.0018$ & $0.0365 \pm 0.0010$ & $0.250 \pm 0.011$ & & $231.2 \pm 6.1$ & $226.9 \pm 10.1$ & $183 \pm 6.6$ \\
\hline $0629-3-27$ & 1.01 & n.d & $0.0549 \pm 0.0019$ & $0.0365 \pm 0.0007$ & $0.276 \pm 0.011$ & -0.8 & $231.2 \pm 4.7$ & $247.7 \pm 9.8$ & $407 \pm 13.8$ \\
\hline $0629-3-11$ & 0.84 & 0.01 & $0.0559 \pm 0.0019$ & $0.0371 \pm 0.0014$ & $0.286 \pm 0.015$ & & $234.7 \pm 9.0$ & $255.4 \pm 13.2$ & $451 \pm 15.7$ \\
\hline $0629-3-59$ & 1.60 & n.d & $0.0537 \pm 0.0027$ & $0.0371 \pm 0.0011$ & $0.275 \pm 0.016$ & & $234.9 \pm 6.9$ & $246.4 \pm 14.5$ & $358 \pm 18.3$ \\
\hline $0629-3-56$ & 1.27 & n.d & $0.0501 \pm 0.0020$ & $0.0405 \pm 0.0012$ & $0.280 \pm 0.014$ & & $256.0 \pm 7.4$ & $250.5 \pm 12.4$ & $200 \pm 8.1$ \\
\hline $0629-3-12$ & 0.26 & 0.27 & $0.0515 \pm 0.0018$ & $0.0415 \pm 0.0016$ & $0.294 \pm 0.015$ & & $261.9 \pm 10.0$ & $262.0 \pm 13.5$ & $263 \pm 9.1$ \\
\hline $0629-3-34$ & 0.19 & n.d & $0.0572 \pm 0.0044$ & $0.0417 \pm 0.0010$ & $0.329 \pm 0.027$ & & $263.6 \pm 6.1$ & $288.9 \pm 23.3$ & $499 \pm 38.6$ \\
\hline $0629-3-91$ & 0.19 & n.d & $0.0486 \pm 0.0073$ & $0.0434 \pm 0.0012$ & $0.291 \pm 0.044$ & & $273.7 \pm 7.3$ & $259.1 \pm 39.4$ & $129 \pm 19.3$ \\
\hline $0629-3-87$ & 0.91 & 0.60 & $0.0603 \pm 0.0023$ & $0.0586 \pm 0.0018$ & $0.487 \pm 0.024$ & -1.3 & $366.9 \pm 11.6$ & $403.0 \pm 20.0$ & $617 \pm 23.7$ \\
\hline $0629-3-18$ & 0.74 & n.d & $0.0570 \pm 0.0019$ & $0.0680 \pm 0.0026$ & $0.534 \pm 0.027$ & & $424.0 \pm 16.2$ & $434.7 \pm 21.9$ & $492 \pm 16.1$ \\
\hline $0629-3-88$ & 0.57 & n.d & $0.0555 \pm 0.0024$ & $0.0685 \pm 0.0022$ & $0.524 \pm 0.028$ & & $427.2 \pm 13.5$ & $427.7 \pm 22.7$ & $431 \pm 18.4$ \\
\hline $0629-3-92$ & 0.63 & n.d & $0.0556 \pm 0.0021$ & $0.0712 \pm 0.0015$ & $0.546 \pm 0.024$ & & $443.5 \pm 9.3$ & $442.2 \pm 19.3$ & $436 \pm 16.6$ \\
\hline $0629-3-97$ & 1.12 & 0.73 & $0.0640 \pm 0.0013$ & $0.0817 \pm 0.0016$ & $0.720 \pm 0.020$ & -3.8 & $506.0 \pm 10.1$ & $550.9 \pm 15.6$ & $742 \pm 14.7$ \\
\hline $0629-3-83$ & 1.04 & n.d & $0.0761 \pm 0.0048$ & $0.1109 \pm 0.0037$ & $1.163 \pm 0.083$ & -4.0 & $677.9 \pm 22.6$ & $783.5 \pm 55.6$ & $1098 \pm 69$ \\
\hline $0629-3-64$ & 1.34 & n.d & $0.0656 \pm 0.0016$ & $0.1156 \pm 0.0050$ & $1.045 \pm 0.052$ & & $705.3 \pm 30.3$ & $726.5 \pm 36.0$ & $793 \pm 19.5$ \\
\hline 0629-3-68 & 0.93 & n.d & $0.0655 \pm 0.0016$ & $0.1243 \pm 0.0053$ & $1.123 \pm 0.055$ & & $755.4 \pm 32.4$ & $764.4 \pm 37.6$ & $791 \pm 19.0$ \\
\hline $0629-3-3$ & 0.62 & 0.33 & $0.0778 \pm 0.0012$ & $0.1349 \pm 0.0063$ & $1.447 \pm 0.071$ & -1.3 & $815.5 \pm 37.8$ & $908.4 \pm 44.4$ & $1142 \pm 18$ \\
\hline $0629-3-5$ & 0.80 & n.d & $0.0635 \pm 0.0030$ & $0.1375 \pm 0.0066$ & $1.203 \pm 0.080$ & & $830.4 \pm 39.6$ & $802.1 \pm 53.4$ & $725 \pm 33.7$ \\
\hline
\end{tabular}


Table 1. Continued

\begin{tabular}{|c|c|c|c|c|c|c|c|c|c|}
\hline $\begin{array}{l}\text { Sample } \\
\text { Code }\end{array}$ & $\mathrm{Th} / \mathrm{U}$ & $\begin{array}{c}{ }^{206} \mathrm{Pbc}^{(\mathrm{a}} \\
(\%)\end{array}$ & ${ }^{207} \mathrm{~Pb} /{ }^{206} \mathrm{~Pb}$ & $\begin{array}{cc}{ }^{206} \mathrm{~Pb} /{ }^{238} \mathrm{U} & \begin{array}{c}\text { Error } \\
2 \sigma\end{array}\end{array}$ & $\begin{array}{c}{ }^{207} \mathrm{~Pb} /{ }^{235} \mathrm{U} \\
\begin{array}{c}\text { Error } \\
2 \sigma\end{array}\end{array}$ & $\begin{array}{c}\operatorname{Disc}^{(b)} \\
(\%)\end{array}$ & $\begin{array}{cc}{ }^{238} \mathrm{U}-{ }^{206} \mathrm{~Pb} & \text { Error } \\
\text { age }(\mathrm{Ma}) & 2 \sigma\end{array}$ & $\begin{array}{cc}{ }^{235} \mathrm{U}^{207} \mathrm{~Pb} & \text { Error } \\
\text { age }(\mathrm{Ma}) & 2 \sigma\end{array}$ & $\begin{array}{c}{ }^{207} \mathrm{~Pb}-{ }^{206} \mathrm{~Pb} \text { Error } \\
\text { age (Ma) } \quad 2 \sigma\end{array}$ \\
\hline $0629-3-4$ & 0.92 & n.d & $0.0646 \pm 0.0024$ & $0.1427 \pm 0.0067$ & $1.272 \pm 0.076$ & & $860.0 \pm 40.6$ & $833.2 \pm 49.7$ & $763 \pm 27.9$ \\
\hline $0629-3-33$ & 0.69 & 0.66 & $0.0788 \pm 0.0019$ & $0.1875 \pm 0.0038$ & $2.038 \pm 0.064$ & -1.0 & $1108 \pm 22$ & $1128 \pm 35$ & $1169 \pm 28$ \\
\hline $0629-3-70$ & 0.84 & 0.09 & $0.0817 \pm 0.0017$ & $0.1892 \pm 0.0081$ & $2.131 \pm 0.102$ & -4.2 & $1117 \pm 48$ & $1159 \pm 55$ & $1238 \pm 26$ \\
\hline $0629-3-95$ & 0.60 & n.d & $0.0820 \pm 0.0020$ & $0.2019 \pm 0.0041$ & $2.283 \pm 0.072$ & -0.5 & $1186 \pm 24$ & $1207 \pm 38$ & $1246 \pm 30$ \\
\hline 0629-3-99 & 0.71 & n.d & $0.0790 \pm 0.0019$ & $0.2068 \pm 0.0042$ & $2.252 \pm 0.071$ & & $1212 \pm 25$ & $1197 \pm 38$ & $1172 \pm 28$ \\
\hline $0629-3-52$ & 0.81 & n.d & $0.1125 \pm 0.0019$ & $0.2824 \pm 0.0078$ & $4.381 \pm 0.143$ & -10.0 & $1604 \pm 44$ & $1709 \pm 56$ & $1841 \pm 32$ \\
\hline $0629-3-20$ & 0.77 & 0.03 & $0.1020 \pm 0.0020$ & $0.2834 \pm 0.0107$ & $3.986 \pm 0.169$ & & $1609 \pm 61$ & $1631 \pm 69$ & $1661 \pm 32$ \\
\hline $0629-3-8$ & 0.77 & 0.09 & $0.1096 \pm 0.0017$ & $0.2859 \pm 0.0133$ & $4.323 \pm 0.212$ & -4.3 & $1621 \pm 75$ & $1698 \pm 83$ & $1794 \pm 28$ \\
\hline $0629-3-46$ & 1.28 & n.d & $0.1082 \pm 0.0022$ & $0.2888 \pm 0.0052$ & $4.309 \pm 0.116$ & -4.2 & $1636 \pm 29$ & $1695 \pm 46$ & $1770 \pm 36$ \\
\hline $0629-3-96$ & 0.10 & 0.11 & $0.1139 \pm 0.0019$ & $0.2901 \pm 0.0058$ & $4.555 \pm 0.117$ & -9.6 & $1642 \pm 33$ & $1741 \pm 45$ & $1863 \pm 31$ \\
\hline $0629-3-79$ & 1.14 & 0.55 & $0.1091 \pm 0.0024$ & $0.2912 \pm 0.0076$ & $4.380 \pm 0.149$ & -3.4 & $1647 \pm 43$ & $1709 \pm 58$ & $1785 \pm 39$ \\
\hline $0629-3-32$ & 0.43 & n.d & $0.1082 \pm 0.0022$ & $0.2915 \pm 0.0058$ & $4.350 \pm 0.125$ & -3.1 & $1649 \pm 33$ & $1703 \pm 49$ & $1770 \pm 36$ \\
\hline $0629-3-40$ & 0.07 & 0.81 & $0.1204 \pm 0.0016$ & $0.2935 \pm 0.0057$ & $4.873 \pm 0.113$ & -14.9 & $1659 \pm 32$ & $1798 \pm 42$ & $1963 \pm 26$ \\
\hline 0629-3-69 & 0.27 & n.d & $0.1139 \pm 0.0022$ & $0.2949 \pm 0.0126$ & $4.633 \pm 0.217$ & -5.4 & $1666 \pm 71$ & $1755 \pm 82$ & $1863 \pm 36$ \\
\hline $0629-3-26$ & 0.65 & n.d & $0.1129 \pm 0.0018$ & $0.2959 \pm 0.0058$ & $4.606 \pm 0.116$ & -6.8 & $1671 \pm 33$ & $1750 \pm 44$ & $1847 \pm 29$ \\
\hline $0629-3-24$ & 1.34 & n.d & $0.1062 \pm 0.0028$ & $0.2979 \pm 0.0063$ & $4.361 \pm 0.149$ & & $1681 \pm 36$ & $1705 \pm 58$ & $1735 \pm 46$ \\
\hline $0629-3-23$ & 0.91 & n.d & $0.1166 \pm 0.0019$ & $0.2979 \pm 0.0059$ & $4.788 \pm 0.121$ & -9.6 & $1681 \pm 33$ & $1783 \pm 45$ & $1905 \pm 30$ \\
\hline $0629-3-74$ & 0.15 & n.d & $0.1117 \pm 0.0019$ & $0.3014 \pm 0.0077$ & $4.641 \pm 0.143$ & -3.2 & $1698 \pm 44$ & $1757 \pm 54$ & $1827 \pm 31$ \\
\hline $0629-3-38$ & 1.01 & n.d & $0.1068 \pm 0.0045$ & $0.3033 \pm 0.0070$ & $4.465 \pm 0.215$ & & $1708 \pm 39$ & $1724 \pm 83$ & $1746 \pm 74$ \\
\hline $0629-3-58$ & 0.63 & 0.15 & $0.1116 \pm 0.0018$ & $0.3043 \pm 0.0084$ & $4.684 \pm 0.150$ & -2.1 & $1713 \pm 47$ & $1764 \pm 57$ & $1826 \pm 30$ \\
\hline $0629-3-90$ & 0.85 & 0.03 & $0.1112 \pm 0.0031$ & $0.3055 \pm 0.0096$ & $4.683 \pm 0.198$ & & $1719 \pm 54$ & $1764 \pm 75$ & $1819 \pm 51$ \\
\hline $0629-3-44$ & 0.83 & n.d & $0.1139 \pm 0.0015$ & $0.3072 \pm 0.0053$ & $4.823 \pm 0.104$ & -4.8 & $1727 \pm 30$ & $1789 \pm 39$ & $1863 \pm 24$ \\
\hline $0629-3-78$ & 0.80 & 0.30 & $0.1097 \pm 0.0019$ & $0.3090 \pm 0.0079$ & $4.674 \pm 0.145$ & & $1736 \pm 45$ & $1763 \pm 55$ & $1795 \pm 31$ \\
\hline $0629-3-80$ & 0.35 & 0.04 & $0.1145 \pm 0.0020$ & $0.3118 \pm 0.0080$ & $4.922 \pm 0.152$ & -2.6 & $1750 \pm 45$ & $1806 \pm 56$ & $1872 \pm 32$ \\
\hline $0629-3-41$ & 0.21 & 0.29 & $0.1135 \pm 0.0014$ & $0.3144 \pm 0.0054$ & $4.921 \pm 0.104$ & -2.3 & $1763 \pm 30$ & $1806 \pm 38$ & $1857 \pm 23$ \\
\hline 0629-3-9 & 0.27 & n.d & $0.1142 \pm 0.0018$ & $0.3215 \pm 0.0149$ & $5.061 \pm 0.248$ & & $1797 \pm 83$ & $1830 \pm 90$ & $1867 \pm 30$ \\
\hline $0629-3-17$ & 0.64 & 0.09 & $0.1160 \pm 0.0021$ & $0.3280 \pm 0.0124$ & $5.247 \pm 0.220$ & & $1829 \pm 69$ & $1860 \pm 78$ & $1896 \pm 35$ \\
\hline $0629-3-49$ & 0.44 & n.d & $0.1154 \pm 0.0014$ & $0.3316 \pm 0.0057$ & $5.279 \pm 0.110$ & & $1846 \pm 32$ & $1865 \pm 39$ & $1887 \pm 23$ \\
\hline $0629-3-93$ & 0.29 & n.d & $0.1215 \pm 0.0021$ & $0.3347 \pm 0.0067$ & $5.607 \pm 0.148$ & -2.5 & $1861 \pm 37$ & $1917 \pm 51$ & $1979 \pm 34$ \\
\hline $0629-3-7$ & 0.53 & 0.19 & $0.1073 \pm 0.0017$ & $0.3379 \pm 0.0157$ & $4.998 \pm 0.245$ & & $1877 \pm 87$ & $1819 \pm 89$ & $1754 \pm 28$ \\
\hline $0629-3-28$ & 1.23 & n.d & $0.1131 \pm 0.0014$ & $0.3397 \pm 0.0066$ & $5.298 \pm 0.123$ & & $1885 \pm 37$ & $1869 \pm 43$ & $1851 \pm 24$ \\
\hline $0629-3-76$ & 0.66 & 0.39 & $0.1090 \pm 0.0019$ & $0.3429 \pm 0.0088$ & $5.153 \pm 0.161$ & & $1901 \pm 49$ & $1845 \pm 58$ & $1783 \pm 32$ \\
\hline $0629-3-50$ & 0.56 & n.d & $0.1194 \pm 0.0017$ & $0.3474 \pm 0.0060$ & $5.720 \pm 0.130$ & & $1922 \pm 33$ & $1934 \pm 44$ & $1948 \pm 28$ \\
\hline $0629-3-43$ & 0.15 & n.d & $0.1145 \pm 0.0014$ & $0.3477 \pm 0.0059$ & $5.487 \pm 0.115$ & & $1924 \pm 33$ & $1899 \pm 40$ & $1872 \pm 23$ \\
\hline $0629-3-67$ & 0.21 & 0.09 & $0.1322 \pm 0.0024$ & $0.3496 \pm 0.0149$ & $6.373 \pm 0.297$ & -3.8 & $1932 \pm 83$ & $2028 \pm 94$ & $2128 \pm 39$ \\
\hline $0629-3-30$ & 0.13 & 0.02 & $0.1380 \pm 0.0015$ & $0.3541 \pm 0.0068$ & $6.736 \pm 0.150$ & -9.5 & $1954 \pm 38$ & $2077 \pm 46$ & $2202 \pm 25$ \\
\hline $0629-3-86$ & 1.58 & 0.05 & $0.1479 \pm 0.0030$ & $0.3566 \pm 0.0109$ & $7.273 \pm 0.267$ & -12.7 & $1966 \pm 60$ & $2145 \pm 79$ & $2323 \pm 47$ \\
\hline $0629-3-48$ & 0.12 & 0.02 & $0.1443 \pm 0.0015$ & $0.3666 \pm 0.0062$ & $7.293 \pm 0.143$ & -10.4 & $2013 \pm 34$ & $2148 \pm 42$ & $2280 \pm 23$ \\
\hline $0629-3-63$ & 0.41 & 0.21 & $0.1528 \pm 0.0030$ & $0.3687 \pm 0.0158$ & $7.768 \pm 0.366$ & -10.9 & $2023 \pm 87$ & $2204 \pm 104$ & $2378 \pm 46$ \\
\hline $0629-3-94$ & 0.41 & 0.14 & $0.1713 \pm 0.0029$ & $0.4157 \pm 0.0083$ & $9.820 \pm 0.256$ & -10.8 & $2241 \pm 45$ & $2418 \pm 63$ & $2571 \pm 43$ \\
\hline $0629-3-39$ & 0.70 & 0.54 & $0.1735 \pm 0.0024$ & $0.4171 \pm 0.0081$ & $9.978 \pm 0.238$ & -11.8 & $2247 \pm 44$ & $2433 \pm 58$ & $2592 \pm 36$ \\
\hline $0629-3-14$ & 0.43 & 0.59 & $0.1616 \pm 0.0032$ & $0.4476 \pm 0.0170$ & $9.976 \pm 0.428$ & & $2385 \pm 91$ & $2432 \pm 104$ & $2473 \pm 49$ \\
\hline $0629-3-60$ & 0.69 & 0.57 & $0.1951 \pm 0.0032$ & $0.4512 \pm 0.0125$ & $12.139 \pm 0.391$ & -11.4 & $2401 \pm 67$ & $2615 \pm 84$ & $2786 \pm 46$ \\
\hline \multicolumn{10}{|c|}{ Southern Hasandong Fm. $(n=49)$} \\
\hline hsd2-22 & 0.53 & n.d & $0.0505 \pm 0.0022$ & $0.0171 \pm 0.0005$ & $0.119 \pm 0.006$ & & $109.0 \pm 3.4$ & $114.1 \pm 6.1$ & $221 \pm 9.6$ \\
\hline hsd2-1 & 0.52 & n.d & $0.0476 \pm 0.0015$ & $0.0174 \pm 0.0010$ & $0.114 \pm 0.007$ & & $111.2 \pm 6.1$ & $109.7 \pm 7.0$ & $78 \pm 2.5$ \\
\hline hsd2-24 & 0.52 & n.d & $0.0468 \pm 0.0023$ & $0.0174 \pm 0.0005$ & $0.112 \pm 0.007$ & & $111.4 \pm 3.5$ & $108.2 \pm 6.3$ & $38 \pm 1.9$ \\
\hline
\end{tabular}


Table 1. Continued

\begin{tabular}{|c|c|c|c|c|c|c|c|c|c|}
\hline $\begin{array}{l}\text { Sample } \\
\text { Code }\end{array}$ & $\overline{\mathrm{Th} / \mathrm{U}^{2}}$ & $\begin{array}{c}{ }^{206} \mathrm{Pbc}^{(\mathrm{a})} \\
(\%)\end{array}$ & ${ }^{207} \mathrm{~Pb} /{ }^{206} \mathrm{~Pb}$ & ${ }^{206} \mathrm{~Pb} /{ }^{238} \mathrm{U}$ & $\begin{array}{c}{ }^{207} \mathrm{~Pb} /{ }^{235} \mathrm{U} \\
\text { Error } \\
2 \sigma\end{array}$ & $\begin{array}{c}\operatorname{Disc}^{(b)} \\
(\%)\end{array}$ & $\begin{array}{cc}{ }^{238} \mathrm{U}-{ }^{206} \mathrm{~Pb} & \text { Error } \\
\text { age }(\mathrm{Ma}) & 2 \sigma\end{array}$ & $\begin{array}{l}{ }^{235} \mathrm{U}^{-207} \mathrm{~Pb} \\
\text { age (Ma) }\end{array}$ & $\begin{array}{c}{ }^{207} \mathrm{~Pb}-{ }^{206} \mathrm{~Pb} \text { Error } \\
\text { age }(\mathrm{Ma}) \quad 2 \sigma\end{array}$ \\
\hline hsd2-25 & 0.57 & n.d & $0.0497 \pm 0.0021$ & $0.0176 \pm 0.0005$ & $0.120 \pm 0.006$ & & $112.2 \pm 3.5$ & $115.5 \pm 6.0$ & $184 \pm 7.6$ \\
\hline hsd2-56 & 0.28 & n.d & $0.0486 \pm 0.0024$ & $0.0185 \pm 0.0009$ & $0.124 \pm 0.009$ & & $118.0 \pm 6.0$ & $118.5 \pm 8.4$ & $129 \pm 6.3$ \\
\hline hsd2-57 & 1.26 & n.d & $0.0497 \pm 0.0021$ & $0.0192 \pm 0.0010$ & $0.131 \pm 0.009$ & & $122.6 \pm 6.2$ & $125.4 \pm 8.3$ & $179 \pm 7.7$ \\
\hline hsd2-44 & 3.04 & n.d & $0.0446 \pm 0.0057$ & $0.0193 \pm 0.0007$ & $0.118 \pm 0.016$ & & $122.9 \pm 4.7$ & $113.7 \pm 15.2$ & \\
\hline hsd2-50 & 1.20 & n.d & $0.0498 \pm 0.0039$ & $0.0193 \pm 0.0010$ & $0.133 \pm 0.012$ & & $123.4 \pm 6.5$ & $126.6 \pm 11.9$ & $186 \pm 14.5$ \\
\hline hsd2-18 & 0.45 & n.d & $0.0522 \pm 0.0021$ & $0.0197 \pm 0.0006$ & $0.142 \pm 0.007$ & & $125.5 \pm 3.9$ & $134.4 \pm 6.8$ & $295 \pm 11.9$ \\
\hline hsd2-48 & 0.96 & n.d & $0.0503 \pm 0.0031$ & $0.0197 \pm 0.0010$ & $0.136 \pm 0.011$ & & $125.7 \pm 6.5$ & $129.9 \pm 10.4$ & $207 \pm 12.6$ \\
\hline hsd2-49 & 0.96 & n.d & $0.0498 \pm 0.0031$ & $0.0261 \pm 0.0013$ & $0.179 \pm 0.014$ & & $166.4 \pm 8.6$ & $167.5 \pm 13.4$ & $184 \pm 11.3$ \\
\hline hsd2-2 & 1.05 & 3.37 & $0.0517 \pm 0.0009$ & $0.0318 \pm 0.0018$ & $0.227 \pm 0.013$ & & $202.1 \pm 11.1$ & $207.6 \pm 12.0$ & $271 \pm 4.8$ \\
\hline hsd2-40 & 1.19 & n.d & $0.0644 \pm 0.0019$ & $0.1213 \pm 0.0038$ & $1.077 \pm 0.046$ & & $738.0 \pm 23.1$ & $742.4 \pm 31.9$ & $756 \pm 22.2$ \\
\hline hsd2-27 & 0.39 & n.d & $0.0694 \pm 0.0019$ & $0.1217 \pm 0.0037$ & $1.165 \pm 0.048$ & & $740.3 \pm 22.6$ & $784.4 \pm 32.6$ & $913 \pm 25.6$ \\
\hline hsd2-65 & 1.03 & 0.24 & $0.0677 \pm 0.0019$ & $0.1222 \pm 0.0034$ & $1.140 \pm 0.045$ & & $743.2 \pm 20.8$ & $772.5 \pm 30.5$ & $859 \pm 23.9$ \\
\hline hsd2-62 & 0.41 & 0.51 & $0.0634 \pm 0.0014$ & $0.1247 \pm 0.0034$ & $1.091 \pm 0.039$ & & $757.8 \pm 20.9$ & $749.0 \pm 26.7$ & $723 \pm 16.3$ \\
\hline hsd2-33 & 1.30 & n.d & $0.0804 \pm 0.0020$ & $0.1254 \pm 0.0068$ & $1.391 \pm 0.083$ & -3.9 & $761.6 \pm 41.1$ & $885.1 \pm 52.6$ & $1209 \pm 30$ \\
\hline hsd2-23 & 0.89 & n.d & $0.0679 \pm 0.0022$ & $0.1279 \pm 0.0040$ & $1.197 \pm 0.054$ & & $776.1 \pm 24.0$ & $799.2 \pm 36.1$ & $865 \pm 28.4$ \\
\hline hsd2-13 & 0.39 & n.d & $0.0669 \pm 0.0014$ & $0.1297 \pm 0.0040$ & $1.197 \pm 0.045$ & & $786.4 \pm 24.4$ & $799.1 \pm 30.0$ & $835 \pm 17.6$ \\
\hline hsd2-12 & 0.55 & 0.30 & $0.0658 \pm 0.0012$ & $0.1336 \pm 0.0041$ & $1.213 \pm 0.044$ & & $808.3 \pm 25.0$ & $806.3 \pm 28.9$ & $801 \pm 14.6$ \\
\hline hsd2-52 & 0.84 & n.d & $0.0636 \pm 0.0025$ & $0.1337 \pm 0.0068$ & $1.173 \pm 0.075$ & & $808.9 \pm 41.2$ & $788.2 \pm 50.5$ & $730 \pm 28.4$ \\
\hline hsd2-53 & 0.54 & 0.24 & $0.0650 \pm 0.0014$ & $0.1347 \pm 0.0068$ & $1.208 \pm 0.066$ & & $814.9 \pm 40.9$ & $804.2 \pm 43.8$ & $775 \pm 16.4$ \\
\hline hsd2-29 & 0.96 & 0.19 & $0.0636 \pm 0.0015$ & $0.1385 \pm 0.0075$ & $1.214 \pm 0.072$ & & $836.3 \pm 45.0$ & $806.9 \pm 47.6$ & $727 \pm 17.4$ \\
\hline hsd2-36 & 0.94 & 0.65 & $0.0673 \pm 0.0026$ & $0.1399 \pm 0.0076$ & $1.299 \pm 0.087$ & & $844.0 \pm 46.0$ & $845.1 \pm 56.6$ & $849 \pm 33.0$ \\
\hline hsd2-45 & 1.22 & n.d & $0.0656 \pm 0.0026$ & $0.1431 \pm 0.0046$ & $1.294 \pm 0.066$ & & $862.4 \pm 27.7$ & $843.2 \pm 43.2$ & $794 \pm 31.8$ \\
\hline hsd2-46 & 0.97 & n.d & $0.0637 \pm 0.0023$ & $0.1458 \pm 0.0046$ & $1.281 \pm 0.062$ & & $877.4 \pm 27.9$ & $837.4 \pm 40.6$ & $734 \pm 26.9$ \\
\hline hsd2-6 & 0.27 & 0.11 & $0.1136 \pm 0.0017$ & $0.2737 \pm 0.0150$ & $4.288 \pm 0.244$ & -11.9 & $1560 \pm 86$ & $1691 \pm 96$ & $1858 \pm 28$ \\
\hline hsd2-10 & 0.11 & 0.19 & $0.1162 \pm 0.0018$ & $0.2906 \pm 0.0090$ & $4.654 \pm 0.161$ & -10.5 & $1645 \pm 51$ & $1759 \pm 61$ & $1898 \pm 30$ \\
\hline hsd2-55 & 0.29 & 0.18 & $0.1127 \pm 0.0016$ & $0.2945 \pm 0.0147$ & $4.574 \pm 0.238$ & -4.2 & $1664 \pm 83$ & $1744 \pm 91$ & $1843 \pm 27$ \\
\hline hsd2-15 & 0.04 & n.d & $0.1149 \pm 0.0018$ & $0.2968 \pm 0.0091$ & $4.704 \pm 0.162$ & -7.3 & $1676 \pm 52$ & $1768 \pm 61$ & $1879 \pm 29$ \\
\hline hsd2-54 & 1.28 & 1.49 & $0.0940 \pm 0.0023$ & $0.2981 \pm 0.0151$ & $3.865 \pm 0.218$ & & $1682 \pm 85$ & $1606 \pm 90$ & $1509 \pm 37$ \\
\hline hsd2-11 & 0.54 & n.d & $0.1138 \pm 0.0019$ & $0.3000 \pm 0.0093$ & $4.705 \pm 0.164$ & -5.2 & $1691 \pm 52$ & $1768 \pm 62$ & $1861 \pm 30$ \\
\hline hsd2-26 & 0.54 & 0.54 & $0.1064 \pm 0.0022$ & $0.3005 \pm 0.0091$ & $4.410 \pm 0.161$ & & $1694 \pm 51$ & $1714 \pm 63$ & $1740 \pm 36$ \\
\hline hsd2-42 & 1.10 & 0.14 & $0.1024 \pm 0.0022$ & $0.3041 \pm 0.0094$ & $4.292 \pm 0.161$ & & $1712 \pm 53$ & $1692 \pm 63$ & $1668 \pm 36$ \\
\hline hsd2-20 & 0.04 & n.d & $0.1170 \pm 0.0023$ & $0.3044 \pm 0.0092$ & $4.912 \pm 0.177$ & -6.4 & $1713 \pm 52$ & $1804 \pm 65$ & $1912 \pm 38$ \\
\hline hsd2-67 & 0.98 & 0.05 & $0.1162 \pm 0.0016$ & $0.3046 \pm 0.0083$ & $4.879 \pm 0.149$ & -6.5 & $1714 \pm 47$ & $1799 \pm 55$ & $1899 \pm 26$ \\
\hline hsd2-8 & 0.01 & 0.08 & $0.1139 \pm 0.0018$ & $0.3111 \pm 0.0096$ & $4.885 \pm 0.170$ & -1.9 & $1746 \pm 54$ & $1800 \pm 63$ & $1863 \pm 30$ \\
\hline hsd2-43 & 1.08 & n.d & $0.1042 \pm 0.0026$ & $0.3148 \pm 0.0098$ & $4.521 \pm 0.181$ & & $1764 \pm 55$ & $1735 \pm 69$ & $1700 \pm 43$ \\
\hline hsd2-5 & 0.41 & 0.03 & $0.1146 \pm 0.0018$ & $0.3149 \pm 0.0173$ & $4.975 \pm 0.284$ & & $1765 \pm 97$ & $1815 \pm 104$ & $1874 \pm 29$ \\
\hline hsd2-34 & 0.98 & n.d & $0.1148 \pm 0.0024$ & $0.3206 \pm 0.0172$ & $5.074 \pm 0.293$ & & $1792 \pm 96$ & $1832 \pm 106$ & $1877 \pm 40$ \\
\hline hsd2-66 & 0.20 & n.d & $0.1139 \pm 0.0015$ & $0.3319 \pm 0.0090$ & $5.211 \pm 0.157$ & & $1847 \pm 50$ & $1854 \pm 56$ & $1863 \pm 24$ \\
\hline hsd2-60 & 0.21 & 0.06 & $0.1242 \pm 0.0016$ & $0.3329 \pm 0.0091$ & $5.701 \pm 0.171$ & -4.9 & $1852 \pm 50$ & $1931 \pm 58$ & $2018 \pm 26$ \\
\hline hsd2-17 & 0.23 & n.d & $0.1337 \pm 0.0022$ & $0.3507 \pm 0.0108$ & $6.463 \pm 0.225$ & -5.9 & $1938 \pm 60$ & $2041 \pm 71$ & $2147 \pm 35$ \\
\hline hsd2-58 & 0.45 & n.d & $0.1448 \pm 0.0019$ & $0.3815 \pm 0.0104$ & $7.616 \pm 0.230$ & -5.6 & $2083 \pm 57$ & $2187 \pm 66$ & $2286 \pm 30$ \\
\hline hsd2-7 & 1.33 & 0.13 & $0.1544 \pm 0.0023$ & $0.4030 \pm 0.0221$ & $8.582 \pm 0.488$ & -2.7 & $2183 \pm 120$ & $2295 \pm 131$ & $2396 \pm 35$ \\
\hline hsd2-14 & 0.79 & 0.02 & $0.1613 \pm 0.0025$ & $0.4290 \pm 0.0132$ & $9.540 \pm 0.328$ & -2.6 & $2301 \pm 71$ & $2391 \pm 82$ & $2470 \pm 38$ \\
\hline hsd2-61 & 1.24 & 0.33 & $0.1595 \pm 0.0022$ & $0.4454 \pm 0.0122$ & $9.798 \pm 0.299$ & & $2375 \pm 65$ & $2416 \pm 74$ & $2451 \pm 33$ \\
\hline hsd2-63 & 0.42 & n.d & $0.1844 \pm 0.0027$ & $0.4678 \pm 0.0129$ & $11.894 \pm 0.370$ & -4.5 & $2474 \pm 68$ & $2596 \pm 81$ & $2693 \pm 39$ \\
\hline hsd2-41 & 0.05 & 0.01 & $0.2793 \pm 0.0057$ & $0.5806 \pm 0.0179$ & $22.362 \pm 0.825$ & -8.5 & $2951 \pm 91$ & $3199 \pm 118$ & $3360 \pm 68$ \\
\hline
\end{tabular}


Table 1. Continued

\begin{tabular}{|c|c|c|c|c|c|c|c|c|c|}
\hline $\begin{array}{l}\text { Sample } \\
\text { Code }\end{array}$ & $\mathrm{Th} / \mathrm{U}^{2}$ & $\begin{array}{c}{ }^{206} \mathrm{Pbc}^{(\mathrm{a})} \\
(\%)\end{array}$ & ${ }^{207} \mathrm{~Pb} /{ }^{206} \mathrm{~Pb}$ & $\begin{array}{c}{ }^{206} \mathrm{~Pb} /{ }^{238} \mathrm{U} \\
\begin{array}{c}\text { Error } \\
2 \sigma\end{array}\end{array}$ & $\begin{array}{c}{ }^{207} \mathrm{~Pb} /{ }^{235} \mathrm{U} \begin{array}{c}\text { Error } \\
2 \sigma\end{array} \\
\end{array}$ & $\begin{array}{c}\operatorname{Disc}^{(b)} \\
(\%)\end{array}$ & $\begin{array}{cc}{ }^{238} \mathrm{U}^{2}{ }^{206} \mathrm{~Pb} & \text { Error } \\
\text { age }(\mathrm{Ma}) & 2 \sigma\end{array}$ & $\begin{array}{cc}{ }^{235} \mathrm{U}^{-207} \mathrm{~Pb} & \text { Error } \\
\text { age }(\mathrm{Ma}) & 2 \sigma\end{array}$ & $\begin{array}{l}{ }^{207} \mathrm{~Pb}-{ }^{206} \mathrm{~Pb} \text { Error } \\
\text { age }(\mathrm{Ma}) \quad 2 \sigma\end{array}$ \\
\hline \multicolumn{10}{|c|}{ Central Hasandong Fm. $(n=77)$} \\
\hline grh-23 & 0.85 & n.d & $0.0505 \pm 0.0013$ & $0.0172 \pm 0.0003$ & $0.120 \pm 0.004$ & & $110.1 \pm 2.0$ & $115.1 \pm 3.6$ & $221 \pm 5.6$ \\
\hline grh-24 & 0.74 & n.d & $0.0488 \pm 0.0028$ & $0.0176 \pm 0.0004$ & $0.119 \pm 0.007$ & & $112.7 \pm 2.4$ & $113.9 \pm 6.9$ & $140 \pm 7.9$ \\
\hline grh-44 & 1.07 & n.d & $0.0477 \pm 0.0028$ & $0.0183 \pm 0.0007$ & $0.121 \pm 0.009$ & & $117.1 \pm 4.7$ & $115.7 \pm 8.2$ & $87 \pm 5.1$ \\
\hline grh-93 & 1.65 & n.d & $0.0435 \pm 0.0037$ & $0.0187 \pm 0.0006$ & $0.112 \pm 0.010$ & & $119.5 \pm 3.9$ & $108.0 \pm 9.7$ & \\
\hline grh-50 & 3.08 & n.d & $0.0554 \pm 0.0055$ & $0.0188 \pm 0.0004$ & $0.143 \pm 0.015$ & & $120.1 \pm 2.5$ & $136.1 \pm 13.9$ & $427 \pm 42.6$ \\
\hline grh-46 & 1.56 & n.d & $0.0562 \pm 0.0041$ & $0.0189 \pm 0.0004$ & $0.146 \pm 0.011$ & -4.4 & $120.6 \pm 2.3$ & $138.7 \pm 10.5$ & $462 \pm 33.8$ \\
\hline grh-55 & 1.20 & n.d & $0.0462 \pm 0.0038$ & $0.0198 \pm 0.0004$ & $0.126 \pm 0.011$ & & $126.6 \pm 2.4$ & $120.8 \pm 10.2$ & $8 \pm 0.7$ \\
\hline grh-71 & 1.07 & n.d & $0.0571 \pm 0.0036$ & $0.0249 \pm 0.0005$ & $0.196 \pm 0.013$ & -5.1 & $158.3 \pm 3.2$ & $181.6 \pm 12.1$ & $497 \pm 31.5$ \\
\hline grh-12 & 0.78 & n.d & $0.0523 \pm 0.0039$ & $0.0249 \pm 0.0008$ & $0.180 \pm 0.015$ & & $158.8 \pm 5.2$ & $167.8 \pm 13.6$ & $298 \pm 22.0$ \\
\hline grh-4 & 0.82 & n.d & $0.0490 \pm 0.0018$ & $0.0254 \pm 0.0008$ & $0.172 \pm 0.008$ & & $161.9 \pm 4.9$ & $160.9 \pm 7.8$ & $147 \pm 5.5$ \\
\hline grh-59 & 0.83 & n.d & $0.0536 \pm 0.0020$ & $0.0257 \pm 0.0005$ & $0.190 \pm 0.008$ & -1.6 & $163.3 \pm 3.1$ & $176.4 \pm 7.3$ & $356 \pm 13.1$ \\
\hline grh-19 & 0.89 & 1.97 & $0.0504 \pm 0.0023$ & $0.0261 \pm 0.0005$ & $0.181 \pm 0.009$ & & $165.8 \pm 3.4$ & $169.0 \pm 8.4$ & $214 \pm 9.6$ \\
\hline grh-81 & 0.59 & n.d & $0.0492 \pm 0.0024$ & $0.0264 \pm 0.0005$ & $0.179 \pm 0.009$ & & $167.8 \pm 3.4$ & $166.9 \pm 8.8$ & $156 \pm 7.5$ \\
\hline grh-84 & 0.48 & 0.38 & $0.0508 \pm 0.0021$ & $0.0265 \pm 0.0005$ & $0.186 \pm 0.008$ & & $168.5 \pm 3.3$ & $172.8 \pm 7.8$ & $234 \pm 9.5$ \\
\hline grh-97 & 1.00 & n.d & $0.0514 \pm 0.0026$ & $0.0272 \pm 0.0007$ & $0.193 \pm 0.011$ & & $172.7 \pm 4.2$ & $178.8 \pm 9.9$ & $260 \pm 13.0$ \\
\hline grh-6 & 0.78 & n.d & $0.0496 \pm 0.0026$ & $0.0334 \pm 0.0010$ & $0.228 \pm 0.014$ & & $211.5 \pm 6.7$ & $208.6 \pm 12.8$ & $176 \pm 9.2$ \\
\hline grh-99 & 0.69 & n.d & $0.0511 \pm 0.0019$ & $0.0345 \pm 0.0008$ & $0.243 \pm 0.011$ & & $218.9 \pm 5.2$ & $221.3 \pm 9.7$ & $247 \pm 9.1$ \\
\hline grh-11 & 0.07 & n.d & $0.0500 \pm 0.0018$ & $0.0354 \pm 0.0011$ & $0.244 \pm 0.012$ & & $224.4 \pm 6.9$ & $221.8 \pm 10.5$ & $195 \pm 7.0$ \\
\hline grh-56 & 0.53 & n.d & $0.0556 \pm 0.0096$ & $0.0358 \pm 0.0010$ & $0.275 \pm 0.048$ & & $226.9 \pm 6.3$ & $246.6 \pm 43.1$ & $438 \pm 75.6$ \\
\hline grh-62 & 0.06 & n.d & $0.0516 \pm 0.0021$ & $0.0359 \pm 0.0007$ & $0.256 \pm 0.011$ & & $227.6 \pm 4.4$ & $231.1 \pm 10.4$ & $267 \pm 10.8$ \\
\hline grh-76 & 0.80 & n.d & $0.0577 \pm 0.0035$ & $0.0365 \pm 0.0008$ & $0.290 \pm 0.018$ & -2.6 & $231.2 \pm 5.0$ & $258.7 \pm 16.5$ & $517 \pm 31.0$ \\
\hline grh-60 & 0.49 & n.d & $0.0581 \pm 0.0031$ & $0.0367 \pm 0.0008$ & $0.294 \pm 0.017$ & -4.0 & $232.5 \pm 4.8$ & $261.7 \pm 15.1$ & $533 \pm 28.8$ \\
\hline grh-39 & 0.04 & n.d & $0.0477 \pm 0.0023$ & $0.0375 \pm 0.0015$ & $0.247 \pm 0.016$ & & $237.3 \pm 9.5$ & $223.9 \pm 14.2$ & $86 \pm 4.2$ \\
\hline grh-22 & 0.54 & n.d & $0.0541 \pm 0.0012$ & $0.0656 \pm 0.0012$ & $0.489 \pm 0.014$ & & $409.8 \pm 7.6$ & $404.4 \pm 11.6$ & $374 \pm 8.2$ \\
\hline grh-100 & 0.36 & n.d & $0.0644 \pm 0.0025$ & $0.0683 \pm 0.0016$ & $0.606 \pm 0.028$ & -5.5 & $425.8 \pm 10.2$ & $481.1 \pm 21.9$ & $755 \pm 29.3$ \\
\hline grh-92 & 1.09 & 0.93 & $0.0641 \pm 0.0024$ & $0.0873 \pm 0.0026$ & $0.771 \pm 0.037$ & & $539.4 \pm 16.1$ & $580.3 \pm 27.8$ & $745 \pm 28.0$ \\
\hline grh-47 & 1.72 & n.d & $0.0649 \pm 0.0029$ & $0.1023 \pm 0.0018$ & $0.916 \pm 0.044$ & & $627.9 \pm 10.9$ & $660.3 \pm 31.4$ & $773 \pm 34.3$ \\
\hline grh-77 & 0.08 & n.d & $0.0701 \pm 0.0019$ & $0.1140 \pm 0.0022$ & $1.102 \pm 0.037$ & -2.9 & $695.7 \pm 13.2$ & $754.3 \pm 25.0$ & $933 \pm 25.4$ \\
\hline grh-18 & 1.65 & n.d & $0.0713 \pm 0.0023$ & $0.1158 \pm 0.0023$ & $1.137 \pm 0.043$ & -3.1 & $706.1 \pm 13.9$ & $771.2 \pm 29.1$ & $965 \pm 31.0$ \\
\hline grh-94 & 1.21 & n.d & $0.0679 \pm 0.0029$ & $0.1201 \pm 0.0036$ & $1.124 \pm 0.059$ & & $730.9 \pm 22.1$ & $765.0 \pm 40.1$ & $867 \pm 37.1$ \\
\hline grh-79 & 0.60 & n.d & $0.0661 \pm 0.0021$ & $0.1221 \pm 0.0024$ & $1.114 \pm 0.042$ & & $742.9 \pm 14.5$ & $760.0 \pm 28.5$ & $811 \pm 25.9$ \\
\hline grh-64 & 0.87 & 0.07 & $0.0651 \pm 0.0018$ & $0.1287 \pm 0.0024$ & $1.154 \pm 0.038$ & & $780.2 \pm 14.4$ & $779.3 \pm 25.4$ & $777 \pm 20.9$ \\
\hline grh-5 & 0.85 & n.d & $0.0674 \pm 0.0018$ & $0.1295 \pm 0.0039$ & $1.203 \pm 0.048$ & & $785.1 \pm 23.4$ & $802.1 \pm 32.0$ & $850 \pm 22.6$ \\
\hline grh-10 & 0.91 & n.d & $0.0675 \pm 0.0029$ & $0.1297 \pm 0.0041$ & $1.206 \pm 0.064$ & & $786.2 \pm 24.6$ & $803.5 \pm 42.4$ & $852 \pm 36.3$ \\
\hline grh-30 & 1.41 & n.d & $0.0691 \pm 0.0026$ & $0.1319 \pm 0.0073$ & $1.258 \pm 0.084$ & & $798.9 \pm 44.0$ & $826.8 \pm 55.4$ & $903 \pm 34.4$ \\
\hline grh-20 & 1.36 & n.d & $0.0647 \pm 0.0025$ & $0.1338 \pm 0.0027$ & $1.192 \pm 0.053$ & & $809.3 \pm 16.5$ & $797.0 \pm 35.2$ & $764 \pm 29.9$ \\
\hline grh-26 & 0.81 & n.d & $0.0755 \pm 0.0029$ & $0.1351 \pm 0.0074$ & $1.407 \pm 0.094$ & & $816.9 \pm 45.0$ & $892.0 \pm 59.6$ & $1084 \pm 41$ \\
\hline grh-29 & 0.14 & n.d & $0.0653 \pm 0.0020$ & $0.1388 \pm 0.0076$ & $1.251 \pm 0.079$ & & $838.1 \pm 45.9$ & $823.8 \pm 51.8$ & $786 \pm 24.2$ \\
\hline grh-52 & 0.96 & n.d & $0.0695 \pm 0.0015$ & $0.1428 \pm 0.0022$ & $1.368 \pm 0.036$ & & $860.4 \pm 13.1$ & $875.2 \pm 22.9$ & $913 \pm 19.4$ \\
\hline grh-70 & 0.67 & n.d & $0.0796 \pm 0.0023$ & $0.2014 \pm 0.0033$ & $2.210 \pm 0.073$ & & $1183 \pm 19$ & $1184 \pm 39$ & $1187 \pm 34$ \\
\hline grh-3 & 0.82 & n.d & $0.0780 \pm 0.0022$ & $0.2051 \pm 0.0062$ & $2.206 \pm 0.091$ & & $1203 \pm 36$ & $1183 \pm 49$ & $1148 \pm 33$ \\
\hline grh-68 & 0.44 & 0.09 & $0.1108 \pm 0.0031$ & $0.2679 \pm 0.0044$ & $4.091 \pm 0.132$ & -13.6 & $1530 \pm 25$ & $1652 \pm 54$ & $1813 \pm 51$ \\
\hline grh-7 & 1.07 & 0.49 & $0.1112 \pm 0.0034$ & $0.2774 \pm 0.0085$ & $4.253 \pm 0.183$ & -8.7 & $1578 \pm 48$ & $1684 \pm 72$ & $1819 \pm 55$ \\
\hline grh-43 & 0.53 & 0.26 & $0.1102 \pm 0.0030$ & $0.2850 \pm 0.0112$ & $4.331 \pm 0.208$ & -4.6 & $1616 \pm 64$ & $1699 \pm 82$ & $1804 \pm 49$ \\
\hline grh-58 & 0.14 & n.d & $0.1171 \pm 0.0029$ & $0.2895 \pm 0.0053$ & $4.676 \pm 0.144$ & -12.0 & $1639 \pm 30$ & $1763 \pm 54$ & $1913 \pm 47$ \\
\hline grh-57 & 0.18 & n.d & $0.1182 \pm 0.0029$ & $0.2994 \pm 0.0055$ & $4.881 \pm 0.151$ & -9.6 & $1689 \pm 31$ & $1799 \pm 55$ & $1930 \pm 48$ \\
\hline
\end{tabular}


Table 1. Continued

\begin{tabular}{|c|c|c|c|c|c|c|c|c|c|}
\hline $\begin{array}{l}\text { Sample } \\
\text { Code }\end{array}$ & $\mathrm{Th} / \mathrm{U}^{2}$ & $\begin{array}{c}{ }^{206} \mathrm{Pbc}^{(\mathrm{a})} \\
(\%)\end{array}$ & ${ }^{207} \mathrm{~Pb} /{ }^{206} \mathrm{~Pb}$ & $\begin{array}{cc}{ }^{206} \mathrm{~Pb} /{ }^{238} \mathrm{U} & \begin{array}{c}\text { Error } \\
2 \sigma\end{array}\end{array}$ & $\begin{array}{c}{ }^{207} \mathrm{~Pb} /{ }^{235} \mathrm{U} \\
\begin{array}{c}\text { Error } \\
2 \sigma\end{array}\end{array}$ & $\begin{array}{c}\operatorname{Disc}^{(b)} \\
(\%)\end{array}$ & $\begin{array}{cc}{ }^{238} \mathrm{U}^{206} \mathrm{~Pb} & \text { Error } \\
\text { age }(\mathrm{Ma}) & 2 \sigma\end{array}$ & $\begin{array}{cc}{ }^{235} \mathrm{U}_{-}{ }^{207} \mathrm{~Pb} & \text { Error } \\
\text { age }(\mathrm{Ma}) & 2 \sigma\end{array}$ & $\begin{array}{c}{ }^{207} \mathrm{~Pb}^{206} \mathrm{~Pb} \text { Error } \\
\text { age }(\mathrm{Ma}) \quad 2 \sigma\end{array}$ \\
\hline grh-86 & 0.25 & 0.18 & $0.1170 \pm 0.0041$ & $0.3023 \pm 0.0090$ & $4.876 \pm 0.223$ & -5.4 & $1703 \pm 50$ & $1798 \pm 82$ & $1911 \pm 66$ \\
\hline grh-38 & 0.04 & 0.08 & $0.1187 \pm 0.0033$ & $0.3030 \pm 0.0119$ & $4.960 \pm 0.238$ & -6.5 & $1706 \pm 67$ & $1813 \pm 87$ & $1938 \pm 53$ \\
\hline grh-37 & 0.16 & 0.15 & $0.1116 \pm 0.0030$ & $0.3049 \pm 0.0120$ & $4.693 \pm 0.224$ & & $1716 \pm 67$ & $1766 \pm 84$ & $1827 \pm 50$ \\
\hline grh-83 & 0.64 & n.d & $0.1130 \pm 0.0027$ & $0.3065 \pm 0.0058$ & $4.776 \pm 0.146$ & -2.8 & $1723 \pm 33$ & $1781 \pm 55$ & $1849 \pm 45$ \\
\hline grh-21 & 0.35 & 0.03 & $0.1132 \pm 0.0013$ & $0.3068 \pm 0.0055$ & $4.789 \pm 0.104$ & -4.3 & $1725 \pm 31$ & $1783 \pm 39$ & $1852 \pm 22$ \\
\hline grh-54 & 1.03 & n.d & $0.1071 \pm 0.0031$ & $0.3077 \pm 0.0052$ & $4.542 \pm 0.153$ & & $1729 \pm 29$ & $1739 \pm 59$ & $1750 \pm 51$ \\
\hline grh-33 & 0.89 & n.d & $0.1037 \pm 0.0039$ & $0.3118 \pm 0.0173$ & $4.459 \pm 0.300$ & & $1750 \pm 97$ & $1723 \pm 116$ & $1692 \pm 64$ \\
\hline grh-98 & 0.43 & 0.11 & $0.1124 \pm 0.0029$ & $0.3119 \pm 0.0072$ & $4.832 \pm 0.166$ & -0.1 & $1750 \pm 40$ & $1790 \pm 62$ & $1839 \pm 47$ \\
\hline grh-89 & 0.82 & 0.11 & $0.1120 \pm 0.0040$ & $0.3157 \pm 0.0094$ & $4.876 \pm 0.226$ & & $1768 \pm 53$ & $1798 \pm 83$ & $1833 \pm 65$ \\
\hline grh-45 & 0.63 & 0.06 & $0.1122 \pm 0.0031$ & $0.3173 \pm 0.0125$ & $4.911 \pm 0.238$ & & $1777 \pm 70$ & $1804 \pm 87$ & $1836 \pm 52$ \\
\hline grh-65 & 0.20 & n.d & $0.1132 \pm 0.0028$ & $0.3177 \pm 0.0058$ & $4.959 \pm 0.154$ & & $1778 \pm 33$ & $1812 \pm 56$ & $1852 \pm 46$ \\
\hline grh-63 & 0.39 & n.d & $0.1303 \pm 0.0033$ & $0.3179 \pm 0.0059$ & $5.711 \pm 0.180$ & -13.3 & $1779 \pm 33$ & $1933 \pm 61$ & $2103 \pm 54$ \\
\hline grh-66 & 0.45 & 0.09 & $0.1125 \pm 0.0031$ & $0.3182 \pm 0.0052$ & $4.935 \pm 0.159$ & & $1781 \pm 29$ & $1808 \pm 58$ & $1840 \pm 51$ \\
\hline grh-74 & 0.52 & 0.03 & $0.1117 \pm 0.0033$ & $0.3190 \pm 0.0053$ & $4.912 \pm 0.165$ & & $1785 \pm 30$ & $1804 \pm 61$ & $1827 \pm 53$ \\
\hline grh-36 & 0.30 & 0.19 & $0.1166 \pm 0.0032$ & $0.3204 \pm 0.0126$ & $5.151 \pm 0.247$ & & $1792 \pm 71$ & $1845 \pm 88$ & $1905 \pm 52$ \\
\hline grh-48 & 0.63 & n.d & $0.1151 \pm 0.0019$ & $0.3216 \pm 0.0048$ & $5.105 \pm 0.116$ & -1.4 & $1798 \pm 27$ & $1837 \pm 42$ & $1882 \pm 32$ \\
\hline grh-51 & 0.56 & 1.27 & $0.1145 \pm 0.0018$ & $0.3229 \pm 0.0048$ & $5.100 \pm 0.110$ & -0.7 & $1804 \pm 27$ & $1836 \pm 40$ & $1873 \pm 29$ \\
\hline grh-28 & 0.04 & n.d & $0.1169 \pm 0.0032$ & $0.3254 \pm 0.0178$ & $5.243 \pm 0.320$ & & $1816 \pm 99$ & $1860 \pm 114$ & $1909 \pm 52$ \\
\hline grh-31 & 0.19 & 0.11 & $0.1164 \pm 0.0032$ & $0.3348 \pm 0.0183$ & $5.374 \pm 0.329$ & & $1861 \pm 102$ & $1881 \pm 115$ & $1903 \pm 52$ \\
\hline grh-15 & 0.70 & n.d & $0.1228 \pm 0.0034$ & $0.3397 \pm 0.0103$ & $5.752 \pm 0.237$ & & $1885 \pm 57$ & $1939 \pm 80$ & $1998 \pm 56$ \\
\hline grh-35 & 0.46 & 0.71 & $0.1130 \pm 0.0032$ & $0.3400 \pm 0.0186$ & $5.297 \pm 0.326$ & & $1887 \pm 103$ & $1868 \pm 115$ & $1848 \pm 52$ \\
\hline grh-72 & 0.45 & 0.11 & $0.1216 \pm 0.0035$ & $0.3447 \pm 0.0057$ & $5.780 \pm 0.190$ & & $1909 \pm 31$ & $1943 \pm 64$ & $1981 \pm 56$ \\
\hline grh-69 & 0.25 & n.d & $0.1198 \pm 0.0035$ & $0.3533 \pm 0.0058$ & $5.836 \pm 0.195$ & & $1950 \pm 32$ & $1952 \pm 65$ & $1954 \pm 57$ \\
\hline grh-78 & 0.39 & 0.09 & $0.1195 \pm 0.0029$ & $0.3534 \pm 0.0067$ & $5.822 \pm 0.179$ & & $1951 \pm 37$ & $1950 \pm 60$ & $1949 \pm 47$ \\
\hline grh-1 & 0.50 & n.d & $0.1393 \pm 0.0034$ & $0.3557 \pm 0.0106$ & $6.832 \pm 0.261$ & -7.4 & $1962 \pm 58$ & $2090 \pm 80$ & $2219 \pm 53$ \\
\hline grh-17 & 1.13 & 0.40 & $0.1450 \pm 0.0020$ & $0.3637 \pm 0.0067$ & $7.269 \pm 0.167$ & -11.0 & $1999 \pm 37$ & $2145 \pm 49$ & $2288 \pm 32$ \\
\hline grh-49 & 0.70 & n.d & $0.1308 \pm 0.0024$ & $0.3715 \pm 0.0057$ & $6.700 \pm 0.159$ & -0.1 & $2036 \pm 31$ & $2073 \pm 49$ & $2109 \pm 38$ \\
\hline grh-73 & 0.56 & n.d & $0.1410 \pm 0.0044$ & $0.3722 \pm 0.0064$ & $7.237 \pm 0.258$ & -4.7 & $2040 \pm 35$ & $2141 \pm 76$ & $2240 \pm 70$ \\
\hline grh-25 & 0.19 & n.d & $0.1436 \pm 0.0015$ & $0.3930 \pm 0.0071$ & $7.781 \pm 0.161$ & -3.5 & $2137 \pm 38$ & $2206 \pm 46$ & $2272 \pm 23$ \\
\hline grh-27 & 0.34 & 0.09 & $0.1578 \pm 0.0042$ & $0.4227 \pm 0.0231$ & $9.199 \pm 0.559$ & & $2273 \pm 124$ & $2358 \pm 143$ & $2433 \pm 65$ \\
\hline grh-96 & 0.58 & n.d & $0.1595 \pm 0.0040$ & $0.4482 \pm 0.0104$ & $9.857 \pm 0.334$ & & $2387 \pm 55$ & $2421 \pm 82$ & $2451 \pm 61$ \\
\hline \multicolumn{10}{|c|}{ Northern Hasandong Fm. $(n=74)$} \\
\hline hsd1-94 & 1.26 & n.d & $0.0483 \pm 0.0058$ & $0.0261 \pm 0.001$ & $0.174 \pm 0.0$ & & $166.3 \pm 5.3$ & $162.8 \pm 20.3$ & $112 \pm 13.5$ \\
\hline hsd1-87 & 2.25 & n.d & $0.0553 \pm 0.0056$ & $0.0267 \pm 0.0008$ & $0.203 \pm 0.021$ & & $169.7 \pm 5.4$ & $188.0 \pm 19.8$ & $425 \pm 42.7$ \\
\hline hsd1-76 & 0.42 & n.d & $0.0529 \pm 0.0015$ & $0.0271 \pm 0.0006$ & $0.198 \pm 0.007$ & -0.3 & $172.2 \pm 3.8$ & $183.0 \pm 6.5$ & $326 \pm 9.2$ \\
\hline hsd1-88 & 0.41 & n.d & $0.0495 \pm 0.0013$ & $0.0271 \pm 0.0007$ & $0.185 \pm 0.007$ & & $172.2 \pm 4.4$ & $172.3 \pm 6.3$ & $174 \pm 4.5$ \\
\hline hsd1-46 & 0.68 & 0.85 & $0.0542 \pm 0.0021$ & $0.0271 \pm 0.0014$ & $0.202 \pm 0.013$ & & $172.2 \pm 8.9$ & $187.1 \pm 12.0$ & $379 \pm 14.6$ \\
\hline hsd1-81 & 1.29 & n.d & $0.0444 \pm 0.0026$ & $0.0273 \pm 0.0007$ & $0.167 \pm 0.011$ & & $173.4 \pm 4.1$ & $156.9 \pm 10.0$ & \\
\hline hsd1-80 & 0.42 & n.d & $0.0504 \pm 0.0013$ & $0.0273 \pm 0.0006$ & $0.190 \pm 0.006$ & & $173.7 \pm 3.8$ & $176.5 \pm 5.8$ & $214 \pm 5.3$ \\
\hline hsd1-93 & 0.31 & n.d & $0.0570 \pm 0.0015$ & $0.0273 \pm 0.001$ & $0.215 \pm 0.0$ & -6.9 & $173.8 \pm 4.5$ & $197.5 \pm 7.2$ & $492 \pm 12.8$ \\
\hline hsd1-97 & 0.13 & n.d & $0.0500 \pm 0.0013$ & $0.0274 \pm 0.001$ & $0.189 \pm 0.0$ & & $174.0 \pm 4.5$ & $175.5 \pm 6.5$ & $196 \pm 5.2$ \\
\hline hsd1-85 & 0.44 & n.d & $0.0507 \pm 0.0012$ & $0.0275 \pm 0.0007$ & $0.192 \pm 0.007$ & & $175.0 \pm 4.4$ & $178.6 \pm 6.1$ & $227 \pm 5.3$ \\
\hline hsd1-90 & 0.32 & n.d & $0.0498 \pm 0.0013$ & $0.0276 \pm 0.0007$ & $0.189 \pm 0.007$ & & $175.4 \pm 4.5$ & $176.2 \pm 6.5$ & $188 \pm 5.0$ \\
\hline hsd1-73 & 0.50 & n.d & $0.0512 \pm 0.0014$ & $0.0276 \pm 0.0006$ & $0.195 \pm 0.007$ & & $175.4 \pm 3.8$ & $180.5 \pm 6.4$ & $249 \pm 6.9$ \\
\hline hsd1-75 & 0.67 & n.d & $0.0552 \pm 0.0013$ & $0.0277 \pm 0.0006$ & $0.210 \pm 0.007$ & -4.6 & $175.9 \pm 3.8$ & $193.9 \pm 6.2$ & $421 \pm 9.8$ \\
\hline hsd1-36 & 0.36 & n.d & $0.0504 \pm 0.0017$ & $0.0277 \pm 0.0014$ & $0.193 \pm 0.012$ & & $176.2 \pm 8.8$ & $178.9 \pm 10.8$ & $215 \pm 7.4$ \\
\hline hsd1-37 & 0.46 & n.d & $0.0488 \pm 0.0014$ & $0.0278 \pm 0.0014$ & $0.187 \pm 0.011$ & & $176.8 \pm 8.8$ & $174.2 \pm 10.1$ & $139 \pm 4.1$ \\
\hline
\end{tabular}


Table 1. Continued

\begin{tabular}{|c|c|c|c|c|c|c|c|c|c|}
\hline $\begin{array}{l}\text { Sample } \\
\text { Code }\end{array}$ & $\mathrm{Th} / \mathrm{U}$ & $\begin{array}{c}{ }^{206} \mathrm{Pbc}^{(\mathrm{a})} \\
(\%)\end{array}$ & ${ }^{207} \mathrm{~Pb} /{ }^{206} \mathrm{~Pb}$ & $\begin{array}{cc}{ }^{206} \mathrm{~Pb} /{ }^{238} \mathrm{U} & \begin{array}{c}\text { Error } \\
2 \sigma\end{array}\end{array}$ & $\begin{array}{c}{ }^{207} \mathrm{~Pb} /{ }^{235} \mathrm{U} \\
\begin{array}{c}\text { Error } \\
2 \sigma\end{array}\end{array}$ & $\begin{array}{c}\operatorname{Disc}^{(b)} \\
(\%)\end{array}$ & $\begin{array}{cc}{ }^{238} \mathrm{U}_{-}{ }^{206} \mathrm{~Pb} & \text { Error } \\
\text { age }(\mathrm{Ma}) & 2 \sigma\end{array}$ & $\begin{array}{cc}{ }^{235} \mathrm{U}^{207} \mathrm{~Pb} & \text { Error } \\
\text { age }(\mathrm{Ma}) & 2 \sigma\end{array}$ & $\begin{array}{c}{ }^{207} \mathrm{~Pb}-{ }^{206} \mathrm{~Pb} \text { Error } \\
\text { age (Ma) } \quad 2 \sigma\end{array}$ \\
\hline hsd1-62 & 0.52 & 0.57 & $0.0478 \pm 0.0013$ & $0.0280 \pm 0.0005$ & $0.185 \pm 0.006$ & & $178.2 \pm 3.1$ & $172.0 \pm 5.5$ & $88 \pm 2.4$ \\
\hline hsd1-64 & 0.45 & n.d & $0.0536 \pm 0.0015$ & $0.0280 \pm 0.0005$ & $0.207 \pm 0.007$ & -2.0 & $178.3 \pm 3.1$ & $191.3 \pm 6.3$ & $355 \pm 9.9$ \\
\hline hsd1-92 & 0.15 & n.d & $0.0494 \pm 0.0021$ & $0.0281 \pm 0.001$ & $0.191 \pm 0.0$ & & $178.6 \pm 4.8$ & $177.7 \pm 9.0$ & $167 \pm 7.2$ \\
\hline hsd1-61 & 0.46 & n.d & $0.0514 \pm 0.0013$ & $0.0284 \pm 0.0010$ & $0.201 \pm 0.009$ & & $180.3 \pm 6.5$ & $186.1 \pm 8.3$ & $261 \pm 6.8$ \\
\hline hsd1-52 & 0.64 & n.d & $0.0470 \pm 0.0025$ & $0.0284 \pm 0.0015$ & $0.184 \pm 0.014$ & & $180.4 \pm 9.4$ & $171.4 \pm 12.8$ & $50 \pm 2.7$ \\
\hline hsd1-9 & 0.69 & n.d & $0.0507 \pm 0.0017$ & $0.0286 \pm 0.0007$ & $0.200 \pm 0.008$ & & $181.7 \pm 4.5$ & $185.0 \pm 7.6$ & $227 \pm 7.5$ \\
\hline hsd1-68 & 0.40 & 0.01 & $0.0521 \pm 0.0017$ & $0.0287 \pm 0.0005$ & $0.206 \pm 0.008$ & & $182.2 \pm 3.2$ & $190.2 \pm 6.9$ & $292 \pm 9.3$ \\
\hline hsd1-14 & 0.60 & n.d & $0.0493 \pm 0.0014$ & $0.0287 \pm 0.0007$ & $0.195 \pm 0.007$ & & $182.6 \pm 4.5$ & $181.1 \pm 6.7$ & $163 \pm 4.5$ \\
\hline hsd1-3 & 0.36 & n.d & $0.0536 \pm 0.0010$ & $0.0288 \pm 0.0005$ & $0.213 \pm 0.006$ & -2.3 & $183.1 \pm 3.3$ & $195.9 \pm 5.2$ & $353 \pm 6.8$ \\
\hline hsd1-15 & 0.35 & n.d & $0.0510 \pm 0.0018$ & $0.0290 \pm 0.0007$ & $0.204 \pm 0.009$ & & $184.3 \pm 4.6$ & $188.5 \pm 8.1$ & $242 \pm 8.4$ \\
\hline hsd1-57 & 0.45 & n.d & $0.0531 \pm 0.0012$ & $0.0290 \pm 0.0011$ & $0.212 \pm 0.009$ & & $184.4 \pm 6.7$ & $195.6 \pm 8.4$ & $333 \pm 7.7$ \\
\hline hsd1-34 & 0.44 & n.d & $0.0503 \pm 0.0017$ & $0.0290 \pm 0.0014$ & $0.201 \pm 0.012$ & & $184.5 \pm 9.2$ & $186.3 \pm 11.2$ & $211 \pm 7.0$ \\
\hline hsd1-4 & 0.54 & 0.52 & $0.0498 \pm 0.0011$ & $0.0291 \pm 0.0005$ & $0.199 \pm 0.006$ & & $184.6 \pm 3.4$ & $184.6 \pm 5.2$ & $184 \pm 3.9$ \\
\hline hsd1-53 & 0.50 & n.d & $0.0551 \pm 0.0020$ & $0.0291 \pm 0.0015$ & $0.221 \pm 0.014$ & & $184.9 \pm 9.5$ & $202.9 \pm 12.8$ & $418 \pm 15.2$ \\
\hline hsd1-72 & 0.55 & n.d & $0.0503 \pm 0.0022$ & $0.0291 \pm 0.0007$ & $0.202 \pm 0.010$ & & $184.9 \pm 4.2$ & $186.6 \pm 9.2$ & $208 \pm 9.1$ \\
\hline hsd1-8 & 0.33 & n.d & $0.0507 \pm 0.0013$ & $0.0292 \pm 0.0007$ & $0.204 \pm 0.007$ & & $185.7 \pm 4.6$ & $188.9 \pm 6.7$ & $230 \pm 5.8$ \\
\hline hsd1-95 & 0.51 & 0.40 & $0.0494 \pm 0.0018$ & $0.0293 \pm 0.001$ & $0.200 \pm 0.0$ & & $186.3 \pm 4.9$ & $184.8 \pm 8.2$ & $165 \pm 5.9$ \\
\hline hsd1-31 & 0.48 & 0.53 & $0.0453 \pm 0.0012$ & $0.0294 \pm 0.0015$ & $0.184 \pm 0.010$ & & $186.9 \pm 9.3$ & $171.3 \pm 9.7$ & \\
\hline hsd1-23 & 1.01 & 0.50 & $0.0541 \pm 0.0014$ & $0.0294 \pm 0.0010$ & $0.219 \pm 0.010$ & & $187.0 \pm 6.6$ & $201.4 \pm 8.9$ & $375 \pm 10.0$ \\
\hline hsd1-42 & 0.32 & n.d & $0.0508 \pm 0.0014$ & $0.0295 \pm 0.0012$ & $0.206 \pm 0.010$ & & $187.2 \pm 7.8$ & $190.4 \pm 9.5$ & $232 \pm 6.2$ \\
\hline hsd1-12 & 0.28 & n.d & $0.0496 \pm 0.0012$ & $0.0295 \pm 0.0007$ & $0.202 \pm 0.007$ & & $187.2 \pm 4.6$ & $186.5 \pm 6.5$ & $179 \pm 4.4$ \\
\hline hsd1-7 & 0.33 & n.d & $0.0501 \pm 0.0012$ & $0.0296 \pm 0.0005$ & $0.204 \pm 0.006$ & & $187.8 \pm 3.5$ & $188.6 \pm 5.6$ & $200 \pm 4.6$ \\
\hline hsd1-20 & 0.36 & n.d & $0.0511 \pm 0.0013$ & $0.0296 \pm 0.0010$ & $0.209 \pm 0.009$ & & $188.1 \pm 6.6$ & $192.3 \pm 8.3$ & $245 \pm 6.1$ \\
\hline hsd1-26 & 0.19 & n.d & $0.0495 \pm 0.0009$ & $0.0297 \pm 0.0010$ & $0.203 \pm 0.008$ & & $188.8 \pm 6.6$ & $187.5 \pm 7.4$ & $173 \pm 3.1$ \\
\hline hsd1-29 & 0.73 & n.d & $0.0521 \pm 0.0034$ & $0.0299 \pm 0.0015$ & $0.215 \pm 0.018$ & & $190.2 \pm 9.6$ & $197.7 \pm 16.4$ & $289 \pm 19.0$ \\
\hline hsd1-25 & 0.59 & 0.08 & $0.0504 \pm 0.0016$ & $0.0301 \pm 0.0011$ & $0.209 \pm 0.010$ & & $191.2 \pm 6.8$ & $193.0 \pm 9.2$ & $215 \pm 6.8$ \\
\hline hsd1-32 & 0.52 & n.d & $0.0503 \pm 0.0026$ & $0.0303 \pm 0.0015$ & $0.210 \pm 0.015$ & & $192.5 \pm 9.6$ & $193.9 \pm 13.9$ & $210 \pm 10.7$ \\
\hline hsd1-33 & 0.64 & n.d & $0.0657 \pm 0.0035$ & $0.0307 \pm 0.0015$ & $0.278 \pm 0.020$ & -13.3 & $194.7 \pm 9.8$ & $248.8 \pm 18.3$ & $796 \pm 42.8$ \\
\hline hsd1-35 & 1.14 & n.d & $0.0507 \pm 0.0029$ & $0.0332 \pm 0.0017$ & $0.232 \pm 0.018$ & & $210.4 \pm 10.6$ & $211.6 \pm 16.0$ & $226 \pm 12.8$ \\
\hline hsd1-77 & 1.35 & n.d & $0.0527 \pm 0.0018$ & $0.0334 \pm 0.0007$ & $0.242 \pm 0.010$ & & $211.7 \pm 4.7$ & $220.5 \pm 8.9$ & $315 \pm 10.6$ \\
\hline hsd1-24 & 1.28 & n.d & $0.0584 \pm 0.0031$ & $0.0344 \pm 0.0013$ & $0.277 \pm 0.018$ & -2.9 & $218.1 \pm 8.0$ & $248.2 \pm 15.9$ & $544 \pm 28.7$ \\
\hline hsd1-99 & 0.33 & 0.65 & $0.0590 \pm 0.0011$ & $0.0344 \pm 0.001$ & $0.280 \pm 0.0$ & -8.6 & $218.1 \pm 5.6$ & $250.5 \pm 8.0$ & $566 \pm 10.9$ \\
\hline hsd1-6 & 0.61 & n.d & $0.0512 \pm 0.0021$ & $0.0347 \pm 0.0007$ & $0.245 \pm 0.011$ & & $220.1 \pm 4.3$ & $222.8 \pm 10.3$ & $252 \pm 10.5$ \\
\hline hsd1-83 & 0.55 & 3.44 & $0.0462 \pm 0.0009$ & $0.0348 \pm 0.0009$ & $0.221 \pm 0.007$ & & $220.6 \pm 5.6$ & $203.1 \pm 6.5$ & $6 \pm 0.1$ \\
\hline hsd1-82 & 1.15 & 0.16 & $0.0528 \pm 0.0011$ & $0.0352 \pm 0.0009$ & $0.256 \pm 0.008$ & & $222.8 \pm 5.6$ & $231.3 \pm 7.5$ & $320 \pm 6.6$ \\
\hline hsd1-96 & 1.38 & n.d & $0.0547 \pm 0.0016$ & $0.0354 \pm 0.001$ & $0.267 \pm 0.0$ & -0.4 & $224.4 \pm 5.8$ & $240.4 \pm 9.3$ & $400 \pm 11.6$ \\
\hline hsd1-44 & 1.92 & n.d & $0.0536 \pm 0.0014$ & $0.0359 \pm 0.0015$ & $0.266 \pm 0.013$ & & $227.5 \pm 9.5$ & $239.3 \pm 11.7$ & $357 \pm 9.0$ \\
\hline hsd1-39 & 0.54 & n.d & $0.0520 \pm 0.0012$ & $0.0359 \pm 0.0015$ & $0.258 \pm 0.012$ & & $227.6 \pm 9.5$ & $232.8 \pm 11.2$ & $286 \pm 6.7$ \\
\hline hsd1-60 & 0.36 & n.d & $0.0515 \pm 0.0015$ & $0.0359 \pm 0.0013$ & $0.255 \pm 0.012$ & & $227.7 \pm 8.3$ & $230.8 \pm 10.6$ & $263 \pm 7.4$ \\
\hline hsd1-91 & 1.24 & n.d & $0.0502 \pm 0.0014$ & $0.0360 \pm 0.0009$ & $0.250 \pm 0.010$ & & $228.1 \pm 5.9$ & $226.2 \pm 8.7$ & $206 \pm 5.9$ \\
\hline hsd1-22 & 0.91 & n.d & $0.0513 \pm 0.0014$ & $0.0369 \pm 0.0013$ & $0.261 \pm 0.012$ & & $233.6 \pm 8.3$ & $235.4 \pm 10.5$ & $254 \pm 6.8$ \\
\hline hsd1-2 & 1.25 & n.d & $0.0500 \pm 0.0009$ & $0.0372 \pm 0.0007$ & $0.256 \pm 0.007$ & & $235.3 \pm 4.3$ & $231.6 \pm 5.9$ & $195 \pm 3.5$ \\
\hline hsd1-43 & 0.15 & n.d & $0.0517 \pm 0.0013$ & $0.0373 \pm 0.0016$ & $0.266 \pm 0.013$ & & $235.9 \pm 9.9$ & $239.2 \pm 11.7$ & $272 \pm 6.9$ \\
\hline hsd1-56 & 0.55 & 0.96 & $0.0544 \pm 0.0011$ & $0.0374 \pm 0.0014$ & $0.280 \pm 0.012$ & & $236.5 \pm 8.5$ & $250.7 \pm 10.3$ & $387 \pm 7.6$ \\
\hline hsd1-41 & 0.92 & n.d & $0.0649 \pm 0.0014$ & $0.0388 \pm 0.0016$ & $0.347 \pm 0.016$ & -13.2 & $245.5 \pm 10.3$ & $302.6 \pm 14.3$ & $770 \pm 16.8$ \\
\hline hsd1-47 & 0.57 & 0.04 & $0.1154 \pm 0.0033$ & $0.2959 \pm 0.0151$ & $4.708 \pm 0.276$ & -4.5 & $1671 \pm 85$ & $1769 \pm 104$ & $1886 \pm 54$ \\
\hline hsd1-28 & 0.78 & 0.21 & $0.1266 \pm 0.0018$ & $0.3032 \pm 0.0150$ & $5.293 \pm 0.272$ & -13.6 & $1707 \pm 85$ & $1868 \pm 96$ & $2052 \pm 28$ \\
\hline
\end{tabular}


Table 1. Continued

\begin{tabular}{|c|c|c|c|c|c|c|c|c|c|}
\hline $\begin{array}{l}\text { Sample } \\
\text { Code }\end{array}$ & $\mathrm{Th} / \mathrm{U}$ & $\begin{array}{c}{ }^{206} \mathrm{Pbc}^{(\mathrm{a})} \\
(\%)\end{array}$ & ${ }^{207} \mathrm{~Pb} /{ }^{206} \mathrm{~Pb}$ & $\begin{array}{cc}{ }^{206} \mathrm{~Pb} /{ }^{238} \mathrm{U} & \begin{array}{c}\text { Error } \\
2 \sigma\end{array}\end{array}$ & $\begin{array}{c}{ }^{207} \mathrm{~Pb} /{ }^{235} \mathrm{U} \begin{array}{c}\text { Error } \\
2 \sigma\end{array} \\
\end{array}$ & $\begin{array}{c}\operatorname{Disc}^{(b)} \\
(\%)\end{array}$ & $\begin{array}{cc}{ }^{238} \mathrm{U}^{2}{ }^{206} \mathrm{~Pb} & \text { Error } \\
\text { age }(\mathrm{Ma}) & 2 \sigma\end{array}$ & $\begin{array}{cc}{ }^{235} \mathrm{U}^{2}{ }^{207} \mathrm{~Pb} & \text { Error } \\
\text { age }(\mathrm{Ma}) & 2 \sigma\end{array}$ & $\begin{array}{c}{ }^{207} \mathrm{~Pb}-{ }^{206} \mathrm{~Pb} \text { Error } \\
\text { age }(\mathrm{Ma}) \quad 2 \sigma\end{array}$ \\
\hline hsd1-40 & 0.52 & 0.10 & $0.1270 \pm 0.0019$ & $0.3073 \pm 0.0128$ & $5.379 \pm 0.239$ & -13.1 & $1727 \pm 72$ & $1881 \pm 84$ & $2057 \pm 31$ \\
\hline hsd1-55 & 0.19 & 1.28 & $0.1144 \pm 0.0019$ & $0.3101 \pm 0.0112$ & $4.893 \pm 0.195$ & -2.1 & $1741 \pm 63$ & $1801 \pm 72$ & $1872 \pm 31$ \\
\hline hsd1-70 & 1.98 & n.d & $0.1144 \pm 0.0027$ & $0.3188 \pm 0.0054$ & $5.030 \pm 0.145$ & -0.8 & $1784 \pm 30$ & $1824 \pm 52$ & $1872 \pm 43$ \\
\hline hsd1-13 & 0.36 & n.d & $0.1158 \pm 0.0018$ & $0.3249 \pm 0.0079$ & $5.188 \pm 0.151$ & -0.3 & $1814 \pm 44$ & $1851 \pm 54$ & $1893 \pm 30$ \\
\hline hsd1-18 & 0.29 & n.d & $0.1248 \pm 0.0018$ & $0.3383 \pm 0.0119$ & $5.823 \pm 0.221$ & -2.8 & $1878 \pm 66$ & $1950 \pm 74$ & $2027 \pm 30$ \\
\hline hsd1-58 & 0.05 & 0.20 & $0.1213 \pm 0.0021$ & $0.3386 \pm 0.0122$ & $5.665 \pm 0.226$ & & $1880 \pm 68$ & $1926 \pm 77$ & $1977 \pm 34$ \\
\hline hsd1-66 & 0.23 & n.d & $0.1330 \pm 0.0029$ & $0.3485 \pm 0.0059$ & $6.389 \pm 0.177$ & -6.8 & $1927 \pm 32$ & $2031 \pm 56$ & $2138 \pm 47$ \\
\hline hsd1-19 & 0.14 & n.d & $0.1220 \pm 0.0017$ & $0.3500 \pm 0.0123$ & $5.890 \pm 0.223$ & & $1935 \pm 68$ & $1960 \pm 74$ & $1987 \pm 28$ \\
\hline hsd1-71 & 0.37 & n.d & $0.1385 \pm 0.0031$ & $0.3535 \pm 0.0060$ & $6.749 \pm 0.190$ & -9.0 & $1951 \pm 33$ & $2079 \pm 58$ & $2209 \pm 50$ \\
\hline hsd1-1 & 0.90 & 0.21 & $0.1662 \pm 0.0018$ & $0.4135 \pm 0.0075$ & $9.477 \pm 0.200$ & -9.9 & $2231 \pm 40$ & $2385 \pm 50$ & $2520 \pm 27$ \\
\hline hsd1-10 & 1.26 & n.d & $0.1625 \pm 0.0024$ & $0.4927 \pm 0.0120$ & $11.043 \pm 0.313$ & & $2582 \pm 63$ & $2527 \pm 72$ & $2483 \pm 36$ \\
\hline hsd1-5 & 0.64 & 0.45 & $0.2724 \pm 0.0028$ & $0.7223 \pm 0.0132$ & $27.132 \pm 0.569$ & & $3505 \pm 64$ & $3388 \pm 71$ & $3321 \pm 34$ \\
\hline \multicolumn{10}{|c|}{ Southern Jinju Fm. $(n=79)$} \\
\hline $\mathrm{jj} 1-35$ & 1.31 & n.d & $0.0499 \pm 0.0028$ & $0.0171 \pm 0.0007$ & $0.118 \pm 0.008$ & & $109.4 \pm 4.3$ & $113.0 \pm 7.8$ & $190 \pm 10.8$ \\
\hline jj1-50 & 1.64 & n.d & $0.0464 \pm 0.0030$ & $0.0171 \pm 0.0007$ & $0.110 \pm 0.008$ & & $109.5 \pm 4.2$ & $105.6 \pm 7.9$ & $19 \pm 1.2$ \\
\hline jj1-18 & 1.08 & n.d & $0.0492 \pm 0.0017$ & $0.0176 \pm 0.0006$ & $0.120 \pm 0.006$ & & $112.7 \pm 4.0$ & $114.7 \pm 5.7$ & $157 \pm 5.5$ \\
\hline jj1-9 & 1.26 & n.d & $0.0490 \pm 0.0024$ & $0.0180 \pm 0.0006$ & $0.121 \pm 0.007$ & & $114.7 \pm 3.5$ & $116.2 \pm 6.7$ & $148 \pm 7.2$ \\
\hline jj1-6 & 0.25 & n.d & $0.0501 \pm 0.0016$ & $0.0180 \pm 0.0002$ & $0.124 \pm 0.004$ & & $115.1 \pm 1.6$ & $119.1 \pm 4.0$ & $201 \pm 6.2$ \\
\hline jj1-38 & 2.09 & 2.26 & $0.0479 \pm 0.0020$ & $0.0180 \pm 0.0011$ & $0.119 \pm 0.009$ & & $115.2 \pm 6.9$ & $114.3 \pm 8.4$ & $95 \pm 4.0$ \\
\hline jj1-96 & 1.35 & n.d & $0.0524 \pm 0.0017$ & $0.0183 \pm 0.0009$ & $0.132 \pm 0.008$ & & $116.7 \pm 5.9$ & $125.9 \pm 7.6$ & $304 \pm 10.0$ \\
\hline jj1-52 & 1.31 & n.d & $0.0508 \pm 0.0057$ & $0.0184 \pm 0.0008$ & $0.129 \pm 0.015$ & & $117.6 \pm 4.9$ & $123.2 \pm 14.7$ & $233 \pm 26.1$ \\
\hline jj1-13 & 1.54 & n.d & $0.0450 \pm 0.0046$ & $0.0190 \pm 0.0007$ & $0.118 \pm 0.013$ & & $121.2 \pm 4.2$ & $113.0 \pm 12.3$ & \\
\hline jj1-91 & 1.93 & n.d & $0.0514 \pm 0.0017$ & $0.0192 \pm 0.0009$ & $0.136 \pm 0.008$ & & $122.5 \pm 5.9$ & $129.5 \pm 7.6$ & $260 \pm 8.6$ \\
\hline jj1-82 & 1.16 & n.d & $0.0567 \pm 0.0031$ & $0.0194 \pm 0.0010$ & $0.151 \pm 0.012$ & -1.3 & $123.8 \pm 6.7$ & $143.2 \pm 11.1$ & $478 \pm 26.4$ \\
\hline jj1-100 & 1.62 & n.d & $0.0639 \pm 0.0034$ & $0.0210 \pm 0.0011$ & $0.185 \pm 0.014$ & -14.0 & $133.8 \pm 6.9$ & $172.2 \pm 12.8$ & $739 \pm 39.8$ \\
\hline jj1-89 & 0.34 & n.d & $0.0496 \pm 0.0021$ & $0.0244 \pm 0.0012$ & $0.167 \pm 0.011$ & & $155.6 \pm 7.5$ & $157.0 \pm 10.1$ & $178 \pm 7.6$ \\
\hline jj1-81 & 0.38 & n.d & $0.0518 \pm 0.0017$ & $0.0256 \pm 0.0014$ & $0.183 \pm 0.011$ & & $163.2 \pm 8.7$ & $170.7 \pm 10.6$ & $277 \pm 9.1$ \\
\hline jj1-41 & 1.48 & n.d & $0.0493 \pm 0.0020$ & $0.0266 \pm 0.0016$ & $0.181 \pm 0.013$ & & $169.1 \pm 10.1$ & $168.7 \pm 12.2$ & $164 \pm 6.5$ \\
\hline jj1-25 & 0.79 & n.d & $0.0471 \pm 0.0017$ & $0.0276 \pm 0.0010$ & $0.179 \pm 0.009$ & & $175.7 \pm 6.3$ & $167.5 \pm 8.5$ & $53 \pm 1.9$ \\
\hline jj1-16 & 0.42 & n.d & $0.0484 \pm 0.0016$ & $0.0286 \pm 0.0009$ & $0.191 \pm 0.008$ & & $181.6 \pm 5.4$ & $177.1 \pm 7.9$ & $118 \pm 3.9$ \\
\hline jj1-63 & 0.33 & n.d & $0.0507 \pm 0.0013$ & $0.0337 \pm 0.0009$ & $0.236 \pm 0.009$ & & $213.7 \pm 5.8$ & $214.8 \pm 8.0$ & $227 \pm 5.8$ \\
\hline jj1-19 & 2.13 & n.d & $0.0496 \pm 0.0017$ & $0.0353 \pm 0.0013$ & $0.242 \pm 0.012$ & & $223.8 \pm 7.9$ & $219.8 \pm 10.7$ & $178 \pm 6.0$ \\
\hline jj1-98 & 0.48 & 0.90 & $0.0535 \pm 0.0017$ & $0.0354 \pm 0.0018$ & $0.261 \pm 0.016$ & & $224.4 \pm 11.3$ & $235.6 \pm 14.0$ & $349 \pm 11.0$ \\
\hline $\mathrm{jj} 1-2$ & 1.06 & n.d & $0.0522 \pm 0.0024$ & $0.0359 \pm 0.0006$ & $0.258 \pm 0.013$ & & $227.6 \pm 3.5$ & $233.4 \pm 11.4$ & $293 \pm 13.5$ \\
\hline jj1-86 & 0.41 & n.d & $0.0563 \pm 0.0026$ & $0.0457 \pm 0.0022$ & $0.355 \pm 0.024$ & & $288.3 \pm 14.0$ & $308.6 \pm 20.6$ & $465 \pm 21.3$ \\
\hline jj1-23 & 0.80 & n.d & $0.0503 \pm 0.0017$ & $0.0508 \pm 0.0018$ & $0.352 \pm 0.017$ & & $319.3 \pm 11.3$ & $306.1 \pm 14.8$ & $208 \pm 6.9$ \\
\hline jj1-3 & 0.68 & n.d & $0.0500 \pm 0.0011$ & $0.0511 \pm 0.0007$ & $0.352 \pm 0.009$ & & $321.5 \pm 4.1$ & $306.5 \pm 7.6$ & $195 \pm 4.1$ \\
\hline jj1-60 & 0.73 & n.d & $0.0552 \pm 0.0020$ & $0.0672 \pm 0.0019$ & $0.511 \pm 0.024$ & & $419.2 \pm 11.7$ & $419.2 \pm 19.3$ & $420 \pm 15.4$ \\
\hline jj1-40 & 1.07 & n.d & $0.0649 \pm 0.0024$ & $0.1121 \pm 0.0067$ & $1.002 \pm 0.071$ & & $684.7 \pm 41.1$ & $705.0 \pm 49.8$ & $771 \pm 28.6$ \\
\hline jj1-32 & 1.05 & 0.01 & $0.0665 \pm 0.0015$ & $0.1166 \pm 0.0044$ & $1.069 \pm 0.047$ & & $711.0 \pm 27.0$ & $738.2 \pm 32.5$ & $822 \pm 18.4$ \\
\hline jj1-72 & 0.97 & n.d & $0.0675 \pm 0.0011$ & $0.1205 \pm 0.0042$ & $1.122 \pm 0.043$ & & $733.5 \pm 25.7$ & $764.0 \pm 29.4$ & $855 \pm 13.5$ \\
\hline jj1-73 & 0.66 & n.d & $0.0633 \pm 0.0022$ & $0.1244 \pm 0.0045$ & $1.086 \pm 0.054$ & & $755.9 \pm 27.1$ & $746.7 \pm 37.4$ & $720 \pm 25.2$ \\
\hline jj1-4 & 1.48 & n.d & $0.0677 \pm 0.0011$ & $0.1321 \pm 0.0016$ & $1.233 \pm 0.025$ & & $799.9 \pm 9.9$ & $815.8 \pm 16.2$ & $860 \pm 13.4$ \\
\hline jj1-74 & 1.53 & n.d & $0.0635 \pm 0.0019$ & $0.1326 \pm 0.0047$ & $1.161 \pm 0.054$ & & $802.9 \pm 28.6$ & $782.3 \pm 36.5$ & $725 \pm 21.8$ \\
\hline jj1-42 & 0.68 & n.d & $0.0675 \pm 0.0014$ & $0.1399 \pm 0.0083$ & $1.302 \pm 0.082$ & & $844.3 \pm 50.3$ & $846.8 \pm 53.3$ & $854 \pm 17.4$ \\
\hline jj1-55 & 0.12 & n.d & $0.0736 \pm 0.0014$ & $0.1447 \pm 0.0054$ & $1.468 \pm 0.061$ & & $871.2 \pm 32.2$ & $917.1 \pm 38.1$ & $1030 \pm 20$ \\
\hline jj1-61 & 0.23 & n.d & $0.0809 \pm 0.0016$ & $0.1745 \pm 0.0048$ & $1.947 \pm 0.066$ & -12.7 & $1037 \pm 28$ & $1097 \pm 37$ & $1221 \pm 24$ \\
\hline
\end{tabular}


Table 1. Continued

\begin{tabular}{|c|c|c|c|c|c|c|c|c|c|}
\hline $\begin{array}{l}\text { Sample } \\
\text { Code }\end{array}$ & $\mathrm{Th} / \mathrm{U}^{2}$ & $\begin{array}{c}{ }^{206} \mathrm{Pbc}^{(\mathrm{a})} \\
(\%)\end{array}$ & ${ }^{207} \mathrm{~Pb} /{ }^{206} \mathrm{~Pb}$ & ${ }^{206} \mathrm{~Pb} /{ }^{238} \mathrm{U}$ & $\begin{array}{c}{ }^{207} \mathrm{~Pb} /{ }^{235} \mathrm{U} \\
\text { Error } \\
2 \sigma\end{array}$ & $\begin{array}{c}\operatorname{Disc}^{(b)} \\
(\%)\end{array}$ & $\begin{array}{cc}{ }^{238} \mathrm{U}^{206} \mathrm{~Pb} & \text { Error } \\
\text { age }(\mathrm{Ma}) & 2 \sigma\end{array}$ & $\begin{array}{cc}{ }^{235} \mathrm{U}_{-}{ }^{207} \mathrm{~Pb} & \text { Error } \\
\text { age }(\mathrm{Ma}) & 2 \sigma\end{array}$ & $\begin{array}{c}{ }^{207} \mathrm{~Pb}-{ }^{206} \mathrm{~Pb} \text { Error } \\
\text { age }(\mathrm{Ma}) \quad 2 \sigma\end{array}$ \\
\hline$\overline{\mathrm{jj} 1-21}$ & 0.92 & n.d & $0.0716 \pm 0.0014$ & $0.1764 \pm 0.0062$ & $1.742 \pm 0.070$ & & $1047 \pm 37$ & $1024 \pm 41$ & $976 \pm 20$ \\
\hline jj1-36 & 0.72 & n.d & $0.0821 \pm 0.0018$ & $0.1827 \pm 0.0070$ & $2.067 \pm 0.090$ & -9.0 & $1081 \pm 41$ & $1138 \pm 50$ & $1247 \pm 27$ \\
\hline jj1-65 & 0.68 & n.d & $0.0798 \pm 0.0024$ & $0.1902 \pm 0.0053$ & $2.091 \pm 0.087$ & -0.1 & $1122 \pm 31$ & $1146 \pm 47$ & $1191 \pm 36$ \\
\hline jj1-53 & 0.66 & 0.14 & $0.0800 \pm 0.0015$ & $0.1912 \pm 0.0071$ & $2.109 \pm 0.088$ & -0.4 & $1128 \pm 42$ & $1152 \pm 48$ & $1197 \pm 23$ \\
\hline jj1-30 & 0.58 & 0.44 & $0.0744 \pm 0.0022$ & $0.1939 \pm 0.0075$ & $1.989 \pm 0.096$ & & $1142 \pm 44$ & $1112 \pm 54$ & $1053 \pm 31$ \\
\hline jj1-10 & 0.92 & n.d & $0.0798 \pm 0.0022$ & $0.2150 \pm 0.0064$ & $2.367 \pm 0.096$ & & $1255 \pm 38$ & $1233 \pm 50$ & $1193 \pm 32$ \\
\hline jj1-94 & 0.95 & n.d & $0.0912 \pm 0.0014$ & $0.2283 \pm 0.0114$ & $2.870 \pm 0.150$ & -2.7 & $1326 \pm 66$ & $1374 \pm 72$ & $1450 \pm 23$ \\
\hline jj1-64 & 0.76 & n.d & $0.0971 \pm 0.0016$ & $0.2535 \pm 0.0069$ & $3.394 \pm 0.107$ & -3.3 & $1457 \pm 39$ & $1503 \pm 47$ & $1570 \pm 25$ \\
\hline jj1-99 & 1.02 & n.d & $0.1133 \pm 0.0012$ & $0.2805 \pm 0.0140$ & $4.380 \pm 0.224$ & -10.0 & $1594 \pm 80$ & $1709 \pm 87$ & $1853 \pm 20$ \\
\hline jj1-34 & 0.17 & 0.16 & $0.1129 \pm 0.0017$ & $0.2885 \pm 0.0109$ & $4.490 \pm 0.182$ & -7.6 & $1634 \pm 62$ & $1729 \pm 70$ & $1847 \pm 28$ \\
\hline jj1-79 & 0.60 & 0.19 & $0.1125 \pm 0.0020$ & $0.2895 \pm 0.0153$ & $4.490 \pm 0.251$ & -5.0 & $1639 \pm 87$ & $1729 \pm 97$ & $1841 \pm 33$ \\
\hline jj1-24 & 0.87 & n.d & $0.1134 \pm 0.0021$ & $0.2908 \pm 0.0102$ & $4.547 \pm 0.179$ & -7.3 & $1645 \pm 58$ & $1740 \pm 69$ & $1856 \pm 34$ \\
\hline jj1-57 & 0.42 & n.d & $0.1135 \pm 0.0017$ & $0.2916 \pm 0.0079$ & $4.562 \pm 0.142$ & -8.1 & $1649 \pm 45$ & $1742 \pm 54$ & $1856 \pm 28$ \\
\hline jj1-48 & 0.77 & n.d & $0.1059 \pm 0.0034$ & $0.2988 \pm 0.0114$ & $4.365 \pm 0.219$ & & $1685 \pm 64$ & $1706 \pm 85$ & $1731 \pm 56$ \\
\hline jj1-77 & 0.70 & 0.13 & $0.1123 \pm 0.0018$ & $0.2988 \pm 0.0158$ & $4.628 \pm 0.255$ & -2.0 & $1686 \pm 89$ & $1754 \pm 97$ & $1838 \pm 29$ \\
\hline jj1-85 & 1.05 & n.d & $0.1156 \pm 0.0027$ & $0.3020 \pm 0.0145$ & $4.813 \pm 0.257$ & -3.6 & $1701 \pm 82$ & $1787 \pm 95$ & $1889 \pm 45$ \\
\hline jj1-80 & 0.54 & 0.05 & $0.1214 \pm 0.0019$ & $0.3057 \pm 0.0161$ & $5.117 \pm 0.282$ & -7.9 & $1720 \pm 91$ & $1839 \pm 101$ & $1977 \pm 31$ \\
\hline jj1-70 & 0.57 & n.d & $0.1167 \pm 0.0016$ & $0.3059 \pm 0.0107$ & $4.922 \pm 0.185$ & -5.8 & $1721 \pm 60$ & $1806 \pm 68$ & $1907 \pm 26$ \\
\hline jj1-59 & 0.41 & 0.03 & $0.1146 \pm 0.0018$ & $0.3074 \pm 0.0083$ & $4.856 \pm 0.153$ & -4.0 & $1728 \pm 47$ & $1795 \pm 57$ & $1874 \pm 30$ \\
\hline jj1-27 & 0.83 & n.d & $0.1134 \pm 0.0022$ & $0.3124 \pm 0.0110$ & $4.883 \pm 0.196$ & -0.3 & $1752 \pm 62$ & $1799 \pm 72$ & $1855 \pm 36$ \\
\hline jj1-75 & 0.69 & n.d & $0.1147 \pm 0.0015$ & $0.3126 \pm 0.0109$ & $4.943 \pm 0.184$ & -2.1 & $1754 \pm 61$ & $1810 \pm 67$ & $1875 \pm 24$ \\
\hline jj1-33 & 0.44 & n.d & $0.1156 \pm 0.0019$ & $0.3129 \pm 0.0119$ & $4.987 \pm 0.206$ & -2.1 & $1755 \pm 66$ & $1817 \pm 75$ & $1889 \pm 31$ \\
\hline jj1-47 & 0.86 & 0.03 & $0.1163 \pm 0.0021$ & $0.3169 \pm 0.0117$ & $5.081 \pm 0.209$ & -1.5 & $1775 \pm 66$ & $1833 \pm 75$ & $1900 \pm 34$ \\
\hline jj1-95 & 0.49 & n.d & $0.1166 \pm 0.0012$ & $0.3185 \pm 0.0159$ & $5.118 \pm 0.260$ & -0.8 & $1782 \pm 89$ & $1839 \pm 94$ & $1905 \pm 19$ \\
\hline jj1-17 & 0.46 & 0.03 & $0.1134 \pm 0.0019$ & $0.3240 \pm 0.0095$ & $5.068 \pm 0.170$ & & $1809 \pm 53$ & $1831 \pm 62$ & $1856 \pm 31$ \\
\hline jj1-22 & 0.23 & n.d & $0.1133 \pm 0.0021$ & $0.3279 \pm 0.0115$ & $5.123 \pm 0.202$ & & $1828 \pm 64$ & $1840 \pm 73$ & $1854 \pm 34$ \\
\hline jj1-49 & 0.77 & n.d & $0.1201 \pm 0.0022$ & $0.3312 \pm 0.0122$ & $5.482 \pm 0.226$ & -0.6 & $1844 \pm 68$ & $1898 \pm 78$ & $1958 \pm 35$ \\
\hline jj1-54 & 0.19 & n.d & $0.1397 \pm 0.0025$ & $0.3318 \pm 0.0123$ & $6.389 \pm 0.262$ & -14.5 & $1847 \pm 68$ & $2031 \pm 83$ & $2223 \pm 39$ \\
\hline jj1-45 & 0.41 & 0.03 & $0.1244 \pm 0.0018$ & $0.3329 \pm 0.0198$ & $5.708 \pm 0.349$ & -1.5 & $1852 \pm 110$ & $1933 \pm 118$ & $2020 \pm 29$ \\
\hline jj1-97 & 0.70 & n.d & $0.1147 \pm 0.0011$ & $0.3332 \pm 0.0166$ & $5.271 \pm 0.268$ & & $1854 \pm 92$ & $1864 \pm 95$ & $1876 \pm 18$ \\
\hline jj1-44 & 0.60 & 0.04 & $0.1131 \pm 0.0018$ & $0.3355 \pm 0.0200$ & $5.235 \pm 0.323$ & & $1865 \pm 111$ & $1858 \pm 115$ & $1851 \pm 30$ \\
\hline jj1-51 & 0.63 & 0.13 & $0.1200 \pm 0.0026$ & $0.3362 \pm 0.0125$ & $5.563 \pm 0.240$ & & $1868 \pm 70$ & $1910 \pm 82$ & $1957 \pm 42$ \\
\hline jj1-71 & 0.11 & n.d & $0.1405 \pm 0.0018$ & $0.3381 \pm 0.0118$ & $6.547 \pm 0.244$ & -13.9 & $1877 \pm 66$ & $2052 \pm 77$ & $2233 \pm 29$ \\
\hline jj1-11 & 0.18 & 0.32 & $0.1119 \pm 0.0019$ & $0.3411 \pm 0.0100$ & $5.263 \pm 0.177$ & & $1892 \pm 55$ & $1863 \pm 63$ & $1831 \pm 30$ \\
\hline jj1-84 & 0.70 & 0.09 & $0.1400 \pm 0.0031$ & $0.3476 \pm 0.0166$ & $6.711 \pm 0.353$ & -8.6 & $1923 \pm 92$ & $2074 \pm 109$ & $2228 \pm 49$ \\
\hline jj1-37 & 0.33 & n.d & $0.1338 \pm 0.0020$ & $0.3489 \pm 0.0132$ & $6.436 \pm 0.261$ & -6.0 & $1929 \pm 73$ & $2037 \pm 83$ & $2149 \pm 32$ \\
\hline jj1-43 & 1.82 & 0.01 & $0.1136 \pm 0.0018$ & $0.3507 \pm 0.0209$ & $5.492 \pm 0.338$ & & $1938 \pm 115$ & $1899 \pm 117$ & $1858 \pm 29$ \\
\hline jj1-39 & 0.23 & 0.15 & $0.1311 \pm 0.0019$ & $0.3593 \pm 0.0214$ & $6.496 \pm 0.398$ & & $1979 \pm 118$ & $2045 \pm 125$ & $2113 \pm 31$ \\
\hline jj1-78 & 0.97 & 0.02 & $0.1275 \pm 0.0021$ & $0.3647 \pm 0.0193$ & $6.411 \pm 0.355$ & & $2005 \pm 106$ & $2034 \pm 113$ & $2064 \pm 34$ \\
\hline jj1-69 & 0.45 & n.d & $0.1583 \pm 0.0024$ & $0.3743 \pm 0.0132$ & $8.169 \pm 0.314$ & -13.6 & $2049 \pm 72$ & $2250 \pm 86$ & $2438 \pm 38$ \\
\hline jj1-46 & 0.50 & 0.02 & $0.1574 \pm 0.0027$ & $0.3838 \pm 0.0142$ & $8.328 \pm 0.340$ & -10.2 & $2094 \pm 77$ & $2267 \pm 92$ & $2428 \pm 42$ \\
\hline jj1-68 & 0.49 & 0.01 & $0.1575 \pm 0.0022$ & $0.4072 \pm 0.0143$ & $8.843 \pm 0.333$ & -5.3 & $2202 \pm 77$ & $2322 \pm 87$ & $2430 \pm 33$ \\
\hline jj1-5 & 0.69 & 0.03 & $0.1517 \pm 0.0023$ & $0.4086 \pm 0.0052$ & $8.546 \pm 0.169$ & -4.2 & $2209 \pm 28$ & $2291 \pm 45$ & $2365 \pm 36$ \\
\hline jj1-12 & 0.43 & 0.18 & $0.1522 \pm 0.0028$ & $0.4113 \pm 0.0121$ & $8.630 \pm 0.298$ & -1.9 & $2221 \pm 65$ & $2300 \pm 79$ & $2371 \pm 43$ \\
\hline jj1-28 & 2.10 & n.d & $0.1584 \pm 0.0030$ & $0.4366 \pm 0.0167$ & $9.538 \pm 0.407$ & & $2336 \pm 89$ & $2391 \pm 102$ & $2440 \pm 47$ \\
\hline \multicolumn{10}{|c|}{ Central Jinju Fm. $(n=79)$} \\
\hline jj2-70 & 0.81 & n.d & $0.0554 \pm 0.0052$ & $0.0166 \pm 0.0006$ & $0.127 \pm 0.013$ & & $106.2 \pm 3.7$ & $121.3 \pm 12.2$ & $430 \pm 40.6$ \\
\hline jj2-87 & 1.26 & n.d & $0.0545 \pm 0.0035$ & $0.0171 \pm 0.0006$ & $0.128 \pm 0.009$ & -0.4 & $109.0 \pm 4.0$ & $122.5 \pm 9.1$ & $394 \pm 25.4$ \\
\hline
\end{tabular}


Table 1. Continued

\begin{tabular}{|c|c|c|c|c|c|c|c|c|c|}
\hline $\begin{array}{l}\text { Sample } \\
\text { Code }\end{array}$ & $\mathrm{Th} / \mathrm{U}^{2}$ & $\begin{array}{c}{ }^{206} \mathrm{Pbc}^{(\mathrm{a})} \\
(\%)\end{array}$ & ${ }^{207} \mathrm{~Pb} /{ }^{206} \mathrm{~Pb}$ & $\begin{array}{cc}{ }^{206} \mathrm{~Pb} /{ }^{238} \mathrm{U} & \begin{array}{c}\text { Error } \\
2 \sigma\end{array}\end{array}$ & $\begin{array}{c}{ }^{207} \mathrm{~Pb} /{ }^{235} \mathrm{U} \\
\begin{array}{c}\text { Error } \\
2 \sigma\end{array}\end{array}$ & $\begin{array}{c}\operatorname{Disc}^{(b)} \\
(\%)\end{array}$ & $\begin{array}{cc}{ }^{238} \mathrm{U}_{-}{ }^{206} \mathrm{~Pb} & \text { Error } \\
\text { age }(\mathrm{Ma}) & 2 \sigma \\
\end{array}$ & $\begin{array}{cc}{ }^{235} \mathrm{U}^{2}{ }^{207} \mathrm{~Pb} & \text { Error } \\
\text { age }(\mathrm{Ma}) & 2 \sigma \\
\end{array}$ & $\begin{array}{l}{ }^{207} \mathrm{~Pb}-{ }^{206} \mathrm{~Pb} \text { Error } \\
\text { age }(\mathrm{Ma}) \quad 2 \sigma\end{array}$ \\
\hline jj2-74 & 2.13 & n.d & $0.0494 \pm 0.0040$ & $0.0173 \pm 0.0004$ & $0.118 \pm 0.010$ & & $110.5 \pm 2.9$ & $113.1 \pm 9.6$ & $169 \pm 13.7$ \\
\hline jj2-51 & 1.19 & n.d & $0.0480 \pm 0.0025$ & $0.0180 \pm 0.0007$ & $0.119 \pm 0.008$ & & $115.0 \pm 4.6$ & $114.3 \pm 7.6$ & $99 \pm 5.3$ \\
\hline jj2-98 & 0.99 & 0.24 & $0.0483 \pm 0.0027$ & $0.0181 \pm 0.0008$ & $0.120 \pm 0.009$ & & $115.6 \pm 5.1$ & $115.4 \pm 8.2$ & $113 \pm 6.3$ \\
\hline jj2-58 & 1.04 & n.d & $0.0495 \pm 0.0040$ & $0.0181 \pm 0.0007$ & $0.124 \pm 0.011$ & & $115.8 \pm 4.7$ & $118.4 \pm 10.8$ & $172 \pm 14.0$ \\
\hline jj2-43 & 2.05 & n.d & $0.0573 \pm 0.0053$ & $0.0187 \pm 0.0006$ & $0.148 \pm 0.015$ & -2.3 & $119.6 \pm 4.0$ & $140.2 \pm 13.8$ & $505 \pm 46.8$ \\
\hline jj2-41 & 1.05 & n.d & $0.0505 \pm 0.0039$ & $0.0190 \pm 0.0006$ & $0.132 \pm 0.011$ & & $121.3 \pm 3.9$ & $126.1 \pm 10.6$ & $218 \pm 16.9$ \\
\hline jj2-26 & 2.13 & n.d & $0.0516 \pm 0.0038$ & $0.0195 \pm 0.0007$ & $0.139 \pm 0.011$ & & $124.6 \pm 4.4$ & $132.0 \pm 10.8$ & $269 \pm 19.8$ \\
\hline jj2-83 & 0.92 & n.d & $0.0514 \pm 0.0021$ & $0.0199 \pm 0.0007$ & $0.141 \pm 0.008$ & & $126.8 \pm 4.4$ & $133.7 \pm 7.2$ & $260 \pm 10.8$ \\
\hline jj2-94 & 0.36 & n.d & $0.0506 \pm 0.0016$ & $0.0263 \pm 0.0011$ & $0.183 \pm 0.010$ & & $167.3 \pm 7.1$ & $170.9 \pm 9.1$ & $221 \pm 7.0$ \\
\hline jj2-49 & 1.37 & n.d & $0.0528 \pm 0.0031$ & $0.0268 \pm 0.0011$ & $0.195 \pm 0.014$ & & $170.7 \pm 6.8$ & $181.0 \pm 12.8$ & $319 \pm 18.5$ \\
\hline jj2-92 & 0.27 & n.d & $0.0543 \pm 0.0020$ & $0.0269 \pm 0.0009$ & $0.202 \pm 0.010$ & & $171.3 \pm 5.9$ & $186.6 \pm 9.4$ & $385 \pm 14.2$ \\
\hline jj2-34 & 1.35 & n.d & $0.0552 \pm 0.0023$ & $0.0284 \pm 0.0009$ & $0.216 \pm 0.011$ & -1.4 & $180.4 \pm 5.5$ & $198.7 \pm 10.3$ & $423 \pm 17.6$ \\
\hline $\mathrm{jj} 2-75$ & 1.04 & n.d & $0.0504 \pm 0.0045$ & $0.0349 \pm 0.0009$ & $0.242 \pm 0.022$ & & $220.9 \pm 6.0$ & $220.4 \pm 20.4$ & $215 \pm 19.0$ \\
\hline jj2-50 & 0.67 & n.d & $0.0468 \pm 0.0022$ & $0.0351 \pm 0.0014$ & $0.227 \pm 0.014$ & & $222.2 \pm 8.8$ & $207.3 \pm 12.7$ & $42 \pm 2.0$ \\
\hline jj2-38 & 1.32 & n.d & $0.0509 \pm 0.0014$ & $0.0351 \pm 0.0010$ & $0.246 \pm 0.010$ & & $222.4 \pm 6.5$ & $223.4 \pm 9.1$ & $235 \pm 6.6$ \\
\hline $\mathrm{jj} 2-44$ & 0.71 & n.d & $0.0552 \pm 0.0018$ & $0.0355 \pm 0.0011$ & $0.270 \pm 0.012$ & -0.3 & $225.1 \pm 6.7$ & $242.9 \pm 10.6$ & $419 \pm 13.3$ \\
\hline jj2-32 & 0.06 & n.d & $0.0533 \pm 0.0021$ & $0.0390 \pm 0.0012$ & $0.286 \pm 0.014$ & & $246.5 \pm 7.5$ & $255.6 \pm 12.6$ & $341 \pm 13.2$ \\
\hline jj2-86 & 0.86 & n.d & $0.0572 \pm 0.0021$ & $0.0609 \pm 0.0021$ & $0.480 \pm 0.024$ & & $381.2 \pm 13.1$ & $398.1 \pm 20.0$ & $498 \pm 18.3$ \\
\hline jj2-96 & 0.85 & n.d & $0.0513 \pm 0.0035$ & $0.0642 \pm 0.0029$ & $0.454 \pm 0.037$ & & $400.9 \pm 17.9$ & $379.9 \pm 30.7$ & $254 \pm 17.1$ \\
\hline $\mathrm{jj} 2-9$ & 1.02 & n.d & $0.0550 \pm 0.0020$ & $0.0643 \pm 0.0036$ & $0.488 \pm 0.033$ & & $401.7 \pm 22.4$ & $403.3 \pm 26.9$ & $413 \pm 15.2$ \\
\hline jj2-54 & 0.06 & n.d & $0.0562 \pm 0.0014$ & $0.0709 \pm 0.0028$ & $0.550 \pm 0.026$ & & $441.7 \pm 17.1$ & $445.0 \pm 20.7$ & $463 \pm 11.9$ \\
\hline $\mathrm{jj} 2-85$ & 0.89 & n.d & $0.0654 \pm 0.0033$ & $0.0861 \pm 0.0031$ & $0.777 \pm 0.048$ & & $532.6 \pm 19.0$ & $583.6 \pm 35.9$ & $788 \pm 39.5$ \\
\hline $\mathrm{jj} 2-6$ & 0.43 & 1.94 & $0.0660 \pm 0.0008$ & $0.0931 \pm 0.0037$ & $0.847 \pm 0.035$ & -0.2 & $573.8 \pm 22.6$ & $622.9 \pm 25.6$ & $806 \pm 9.4$ \\
\hline jj2-17 & 1.59 & n.d & $0.0696 \pm 0.0017$ & $0.1190 \pm 0.0066$ & $1.142 \pm 0.069$ & & $724.8 \pm 40.2$ & $773.4 \pm 46.8$ & $917 \pm 22.1$ \\
\hline jj2-95 & 1.25 & n.d & $0.0671 \pm 0.0024$ & $0.1357 \pm 0.0058$ & $1.255 \pm 0.071$ & & $820.4 \pm 35.3$ & $825.6 \pm 46.5$ & $840 \pm 30.4$ \\
\hline jj2-29 & 0.61 & n.d & $0.0732 \pm 0.0025$ & $0.1378 \pm 0.0042$ & $1.392 \pm 0.064$ & & $832.5 \pm 25.3$ & $885.3 \pm 40.6$ & $1020 \pm 35$ \\
\hline $\mathrm{jj} 2-45$ & 1.16 & 1.06 & $0.0845 \pm 0.0023$ & $0.1499 \pm 0.0044$ & $1.746 \pm 0.070$ & -6.4 & $900.5 \pm 26.6$ & $1026 \pm 41$ & $1304 \pm 36$ \\
\hline jj2-35 & 1.04 & 0.28 & $0.0714 \pm 0.0017$ & $0.1622 \pm 0.0048$ & $1.596 \pm 0.061$ & & $968.9 \pm 28.7$ & $968.6 \pm 37.2$ & $969 \pm 23.6$ \\
\hline jj2-30 & 0.48 & n.d & $0.0799 \pm 0.0021$ & $0.1662 \pm 0.0050$ & $1.831 \pm 0.073$ & & $991.1 \pm 29.6$ & $1,056.7 \pm 42.2$ & $1195 \pm 32$ \\
\hline jj2-19 & 0.71 & n.d & $0.0834 \pm 0.0025$ & $0.1923 \pm 0.0063$ & $2.210 \pm 0.098$ & -6.1 & $1134 \pm 37$ & $1184 \pm 52$ & $1278 \pm 38$ \\
\hline jj2-89 & 0.68 & n.d & $0.0805 \pm 0.0023$ & $0.1953 \pm 0.0067$ & $2.167 \pm 0.097$ & & $1150 \pm 39$ & $1170 \pm 53$ & $1209 \pm 35$ \\
\hline jj2-12 & 0.22 & 0.04 & $0.1140 \pm 0.0015$ & $0.2676 \pm 0.0148$ & $4.206 \pm 0.238$ & -14.9 & $1529 \pm 84$ & $1675 \pm 95$ & $1865 \pm 24$ \\
\hline $\mathrm{jj} 2-3$ & 0.54 & n.d & $0.1117 \pm 0.0014$ & $0.2702 \pm 0.0106$ & $4.161 \pm 0.172$ & -13.0 & $1542 \pm 61$ & $1666 \pm 69$ & $1827 \pm 23$ \\
\hline jj2-88 & 0.16 & 0.37 & $0.1165 \pm 0.0020$ & $0.2722 \pm 0.0091$ & $4.372 \pm 0.166$ & -17.2 & $1552 \pm 52$ & $1707 \pm 65$ & $1904 \pm 33$ \\
\hline jj2-97 & 0.09 & 0.08 & $0.1141 \pm 0.0021$ & $0.2735 \pm 0.0116$ & $4.303 \pm 0.199$ & -13.3 & $1558 \pm 66$ & $1694 \pm 78$ & $1866 \pm 35$ \\
\hline jj2-25 & 0.32 & n.d & $0.1131 \pm 0.0023$ & $0.2738 \pm 0.0088$ & $4.269 \pm 0.161$ & -13.0 & $1560 \pm 50$ & $1687 \pm 64$ & $1850 \pm 37$ \\
\hline jj2-60 & 0.23 & 0.10 & $0.1107 \pm 0.0025$ & $0.2741 \pm 0.0103$ & $4.182 \pm 0.184$ & -9.6 & $1562 \pm 59$ & $1671 \pm 74$ & $1811 \pm 41$ \\
\hline $\mathrm{jj} 2-100$ & 0.03 & 0.02 & $0.1140 \pm 0.0021$ & $0.2802 \pm 0.0119$ & $4.403 \pm 0.204$ & -10.6 & $1592 \pm 67$ & $1713 \pm 79$ & $1864 \pm 35$ \\
\hline jj2-55 & 0.19 & n.d & $0.1132 \pm 0.0020$ & $0.2819 \pm 0.0109$ & $4.399 \pm 0.186$ & -9.8 & $1601 \pm 62$ & $1712 \pm 72$ & $1852 \pm 32$ \\
\hline jj2-14 & 0.21 & 0.01 & $0.1154 \pm 0.0016$ & $0.2820 \pm 0.0156$ & $4.486 \pm 0.256$ & -10.6 & $1601 \pm 88$ & $1728 \pm 99$ & $1886 \pm 27$ \\
\hline jj2-36 & 0.34 & n.d & $0.1140 \pm 0.0028$ & $0.2843 \pm 0.0085$ & $4.470 \pm 0.172$ & -9.8 & $1613 \pm 48$ & $1725 \pm 66$ & $1865 \pm 46$ \\
\hline jj2-62 & 0.29 & 0.32 & $0.1117 \pm 0.0027$ & $0.2973 \pm 0.0113$ & $4.577 \pm 0.206$ & -2.4 & $1678 \pm 64$ & $1745 \pm 79$ & $1827 \pm 44$ \\
\hline jj2-99 & 0.20 & 0.39 & $0.1135 \pm 0.0022$ & $0.3042 \pm 0.0129$ & $4.761 \pm 0.221$ & -2.2 & $1712 \pm 72$ & $1778 \pm 83$ & $1857 \pm 35$ \\
\hline jj2-82 & 1.01 & n.d & $0.1041 \pm 0.0030$ & $0.3072 \pm 0.0065$ & $4.411 \pm 0.158$ & & $1727 \pm 37$ & $1714 \pm 61$ & $1699 \pm 49$ \\
\hline jj2-93 & 0.55 & n.d & $0.1161 \pm 0.0022$ & $0.3074 \pm 0.0130$ & $4.921 \pm 0.229$ & -3.4 & $1728 \pm 73$ & $1806 \pm 84$ & $1897 \pm 37$ \\
\hline jj2-71 & 1.24 & 0.42 & $0.1056 \pm 0.0031$ & $0.3089 \pm 0.0092$ & $4.497 \pm 0.187$ & & $1735 \pm 52$ & $1730 \pm 72$ & $1725 \pm 50$ \\
\hline jj2-84 & 1.12 & n.d & $0.1131 \pm 0.0023$ & $0.3097 \pm 0.0105$ & $4.828 \pm 0.191$ & -0.8 & $1739 \pm 59$ & $1790 \pm 71$ & $1850 \pm 38$ \\
\hline
\end{tabular}


Table 1. Continued

\begin{tabular}{|c|c|c|c|c|c|c|c|c|c|}
\hline $\begin{array}{l}\text { Sample } \\
\text { Code }\end{array}$ & $\mathrm{Th} / \mathrm{U}$ & $\begin{array}{c}{ }^{206} \mathrm{Pbc}^{(\mathrm{a})} \\
(\%)\end{array}$ & ${ }^{207} \mathrm{~Pb} /{ }^{206} \mathrm{~Pb}$ & $\begin{array}{cc}{ }^{206} \mathrm{~Pb} /{ }^{238} \mathrm{U} & \begin{array}{c}\text { Error } \\
2 \sigma\end{array}\end{array}$ & ${ }^{207} \mathrm{~Pb} /{ }^{235} \mathrm{U} \begin{array}{c}\text { Error } \\
2 \sigma\end{array}$ & $\begin{array}{l}\operatorname{Disc}^{(b)} \\
(\%)\end{array}$ & $\begin{array}{cc}{ }^{238} \mathrm{U}_{-}{ }^{206} \mathrm{~Pb} & \text { Error } \\
\text { age }(\mathrm{Ma}) & 2 \sigma\end{array}$ & $\begin{array}{cc}{ }^{235} \mathrm{U}^{207} \mathrm{~Pb} & \text { Error } \\
\text { age }(\mathrm{Ma}) & 2 \sigma\end{array}$ & $\begin{array}{c}{ }^{207} \mathrm{~Pb}-{ }^{206} \mathrm{~Pb} \text { Error } \\
\text { age (Ma) } \quad 2 \sigma\end{array}$ \\
\hline $\mathrm{jj} 2-7$ & 0.84 & n.d & $0.1147 \pm 0.0016$ & $0.3098 \pm 0.0122$ & $4.899 \pm 0.205$ & -2.3 & $1740 \pm 69$ & $1802 \pm 75$ & $1875 \pm 26$ \\
\hline $\mathrm{jj} 2-22$ & 0.48 & n.d & $0.1128 \pm 0.0024$ & $0.3147 \pm 0.0101$ & $4.894 \pm 0.188$ & & $1764 \pm 57$ & $1801 \pm 69$ & $1845 \pm 39$ \\
\hline $\mathrm{jj} 2-78$ & 0.22 & n.d & $0.1163 \pm 0.0026$ & $0.3153 \pm 0.0064$ & $5.057 \pm 0.152$ & -3.2 & $1767 \pm 36$ & $1829 \pm 55$ & $1901 \pm 42$ \\
\hline $\mathrm{jj} 2-4$ & 0.60 & 0.01 & $0.1162 \pm 0.0015$ & $0.3197 \pm 0.0126$ & $5.122 \pm 0.213$ & -0.9 & $1788 \pm 70$ & $1840 \pm 76$ & $1899 \pm 25$ \\
\hline $\mathrm{jj} 2-59$ & 0.45 & n.d & $0.1144 \pm 0.0026$ & $0.3199 \pm 0.0121$ & $5.045 \pm 0.223$ & & $1789 \pm 68$ & $1827 \pm 81$ & $1871 \pm 43$ \\
\hline $\mathrm{jj} 2-39$ & 0.22 & 0.02 & $0.1148 \pm 0.0026$ & $0.3200 \pm 0.0094$ & $5.065 \pm 0.188$ & & $1790 \pm 52$ & $1830 \pm 68$ & $1877 \pm 43$ \\
\hline $\mathrm{jj} 2-90$ & 0.20 & n.d & $0.1149 \pm 0.0020$ & $0.3212 \pm 0.0108$ & $5.090 \pm 0.193$ & & $1795 \pm 60$ & $1834 \pm 70$ & $1880 \pm 33$ \\
\hline $\mathrm{jj} 2-1$ & 0.90 & 0.07 & $0.1153 \pm 0.0015$ & $0.3225 \pm 0.0127$ & $5.127 \pm 0.213$ & & $1802 \pm 71$ & $1841 \pm 77$ & $1886 \pm 25$ \\
\hline $\mathrm{jj} 2-91$ & 0.65 & n.d & $0.1153 \pm 0.0024$ & $0.3249 \pm 0.0110$ & $5.164 \pm 0.205$ & & $1814 \pm 61$ & $1847 \pm 73$ & $1884 \pm 39$ \\
\hline $\mathrm{jj} 2-72$ & 0.25 & 0.03 & $0.1198 \pm 0.0026$ & $0.3261 \pm 0.0094$ & $5.386 \pm 0.194$ & -2.2 & $1819 \pm 53$ & $1883 \pm 68$ & $1954 \pm 42$ \\
\hline $\mathrm{jj} 2-42$ & 0.27 & n.d & $0.1131 \pm 0.0026$ & $0.3311 \pm 0.0097$ & $5.164 \pm 0.192$ & & $1844 \pm 54$ & $1847 \pm 69$ & $1850 \pm 43$ \\
\hline jj2-66 & 0.84 & 0.39 & $0.1365 \pm 0.0030$ & $0.3341 \pm 0.0097$ & $6.288 \pm 0.230$ & -12.0 & $1858 \pm 54$ & $2017 \pm 74$ & $2184 \pm 49$ \\
\hline $\mathrm{jj} 2-57$ & 0.23 & 0.14 & $0.1122 \pm 0.0025$ & $0.3363 \pm 0.0127$ & $5.201 \pm 0.228$ & & $1869 \pm 70$ & $1853 \pm 81$ & $1836 \pm 41$ \\
\hline jj2-64 & 0.15 & 0.15 & $0.1156 \pm 0.0026$ & $0.3370 \pm 0.0127$ & $5.372 \pm 0.237$ & & $1872 \pm 71$ & $1880 \pm 83$ & $1890 \pm 43$ \\
\hline $\mathrm{jj} 2-24$ & 0.40 & n.d & $0.1313 \pm 0.0027$ & $0.3396 \pm 0.0109$ & $6.149 \pm 0.233$ & -6.8 & $1885 \pm 60$ & $1997 \pm 76$ & $2116 \pm 43$ \\
\hline $\mathrm{jj} 2-61$ & 0.67 & 0.43 & $0.1094 \pm 0.0026$ & $0.3477 \pm 0.0132$ & $5.245 \pm 0.235$ & & $1923 \pm 73$ & $1860 \pm 83$ & $1790 \pm 43$ \\
\hline $\mathrm{jj} 2-21$ & 0.57 & n.d & $0.1392 \pm 0.0028$ & $0.3486 \pm 0.0112$ & $6.693 \pm 0.254$ & -9.5 & $1928 \pm 62$ & $2072 \pm 79$ & $2218 \pm 45$ \\
\hline $\mathrm{jj} 2-10$ & 0.75 & 0.11 & $0.1311 \pm 0.0018$ & $0.3554 \pm 0.0196$ & $6.424 \pm 0.366$ & -0.8 & $1960 \pm 108$ & $2035 \pm 116$ & $2113 \pm 29$ \\
\hline $\mathrm{jj} 2-63$ & 0.05 & n.d & $0.1195 \pm 0.0026$ & $0.3610 \pm 0.0136$ & $5.949 \pm 0.259$ & & $1987 \pm 75$ & $1968 \pm 86$ & $1950 \pm 43$ \\
\hline $\mathrm{jj} 2-46$ & 0.78 & 0.12 & $0.1506 \pm 0.0028$ & $0.3632 \pm 0.0141$ & $7.541 \pm 0.323$ & -11.8 & $1997 \pm 77$ & $2178 \pm 93$ & $2353 \pm 43$ \\
\hline $\mathrm{jj} 2-65$ & 0.46 & 0.05 & $0.1372 \pm 0.0030$ & $0.3661 \pm 0.0138$ & $6.926 \pm 0.302$ & -2.9 & $2011 \pm 76$ & $2102 \pm 92$ & $2193 \pm 48$ \\
\hline $\mathrm{jj} 2-52$ & 0.33 & n.d & $0.1299 \pm 0.0022$ & $0.3805 \pm 0.0147$ & $6.818 \pm 0.287$ & & $2079 \pm 80$ & $2088 \pm 88$ & $2098 \pm 35$ \\
\hline $\mathrm{jj} 2-28$ & 0.60 & n.d & $0.1475 \pm 0.0034$ & $0.3874 \pm 0.0115$ & $7.877 \pm 0.294$ & -4.4 & $2111 \pm 62$ & $2217 \pm 83$ & $2318 \pm 53$ \\
\hline $\mathrm{jj} 2-23$ & 0.62 & n.d & $0.1439 \pm 0.0029$ & $0.4043 \pm 0.0129$ & $8.023 \pm 0.302$ & & $2189 \pm 70$ & $2234 \pm 84$ & $2275 \pm 45$ \\
\hline $\mathrm{jj} 2-2$ & 0.68 & 0.18 & $0.1629 \pm 0.0020$ & $0.4063 \pm 0.0160$ & $9.122 \pm 0.375$ & -7.8 & $2198 \pm 87$ & $2350 \pm 97$ & $2486 \pm 30$ \\
\hline jj2-37 & 0.72 & 0.04 & $0.1620 \pm 0.0037$ & $0.4149 \pm 0.0123$ & $9.265 \pm 0.346$ & -5.2 & $2237 \pm 66$ & $2365 \pm 88$ & $2477 \pm 56$ \\
\hline $\mathrm{jj} 2-53$ & 0.52 & n.d & $0.1709 \pm 0.0029$ & $0.4269 \pm 0.0165$ & $10.059 \pm 0.425$ & -6.2 & $2292 \pm 88$ & $2440 \pm 103$ & $2567 \pm 44$ \\
\hline $\mathrm{jj} 2-18$ & 0.49 & n.d & $0.1762 \pm 0.0037$ & $0.4270 \pm 0.0137$ & $10.373 \pm 0.397$ & -8.6 & $2292 \pm 74$ & $2469 \pm 94$ & $2618 \pm 54$ \\
\hline $\mathrm{jj} 2-20$ & 0.86 & 0.36 & $0.1571 \pm 0.0034$ & $0.4464 \pm 0.0144$ & $9.671 \pm 0.374$ & 0.0 & $2379 \pm 77$ & $2404 \pm 93$ & $2425 \pm 52$ \\
\hline $\mathrm{jj} 2-81$ & 0.50 & n.d & $0.1824 \pm 0.0039$ & $0.5095 \pm 0.0102$ & $12.814 \pm 0.373$ & 0.0 & $2654 \pm 53$ & $2666 \pm 78$ & $2675 \pm 57$ \\
\hline \multicolumn{10}{|c|}{ Northern Jinju Fm. $(n=81)$} \\
\hline $0630-9-92$ & 0.85 & n.d & $0.0547 \pm 0.0015$ & $0.0166 \pm 0.0003$ & $0.125 \pm 0.004$ & -7.4 & $106.0 \pm 1.9$ & $119.7 \pm 4.0$ & $402 \pm 11.4$ \\
\hline $0630-9-76$ & 0.65 & 0.43 & $0.0575 \pm 0.0018$ & $0.0249 \pm 0.0010$ & $0.198 \pm 0.010$ & -5.9 & $158.6 \pm 6.1$ & $183.1 \pm 9.1$ & $513 \pm 16.3$ \\
\hline 0630-9-93 & 0.35 & n.d & $0.0485 \pm 0.0017$ & $0.0257 \pm 0.0005$ & $0.172 \pm 0.007$ & & $163.8 \pm 2.9$ & $161.4 \pm 6.4$ & $126 \pm 4.5$ \\
\hline $0630-9-3$ & 0.15 & n.d & $0.0473 \pm 0.0015$ & $0.0264 \pm 0.0009$ & $0.172 \pm 0.008$ & & $167.7 \pm 5.9$ & $161.0 \pm 7.5$ & $64 \pm 2.0$ \\
\hline $0630-9-17$ & 0.62 & 0.70 & $0.0468 \pm 0.0012$ & $0.0265 \pm 0.0003$ & $0.171 \pm 0.005$ & & $168.5 \pm 2.2$ & $160.3 \pm 4.7$ & $41 \pm 1.1$ \\
\hline $0630-9-5$ & 0.49 & 0.06 & $0.0497 \pm 0.0016$ & $0.0270 \pm 0.0009$ & $0.185 \pm 0.009$ & & $171.6 \pm 6.0$ & $172.1 \pm 8.2$ & $179 \pm 5.8$ \\
\hline $0630-9-14$ & 0.32 & n.d & $0.0504 \pm 0.0016$ & $0.0274 \pm 0.0004$ & $0.190 \pm 0.007$ & & $174.5 \pm 2.4$ & $177.0 \pm 6.3$ & $212 \pm 6.9$ \\
\hline 0630-9-19 & 0.35 & n.d & $0.0512 \pm 0.0019$ & $0.0277 \pm 0.0004$ & $0.195 \pm 0.008$ & & $175.8 \pm 2.5$ & $181.0 \pm 7.1$ & $249 \pm 9.1$ \\
\hline $0630-9-60$ & 0.45 & n.d & $0.0546 \pm 0.0019$ & $0.0279 \pm 0.0010$ & $0.210 \pm 0.010$ & -0.1 & $177.2 \pm 6.3$ & $193.3 \pm 9.6$ & $396 \pm 13.7$ \\
\hline 0630-9-91 & 1.01 & n.d & $0.0551 \pm 0.0042$ & $0.0284 \pm 0.0006$ & $0.216 \pm 0.017$ & & $180.4 \pm 4.0$ & $198.3 \pm 15.6$ & $417 \pm 31.5$ \\
\hline 0630-9-79 & 0.28 & n.d & $0.0509 \pm 0.0019$ & $0.0284 \pm 0.0011$ & $0.199 \pm 0.011$ & & $180.5 \pm 7.0$ & $184.5 \pm 10.0$ & $237 \pm 9.0$ \\
\hline $0630-9-90$ & 0.67 & 0.34 & $0.0480 \pm 0.0021$ & $0.0284 \pm 0.0005$ & $0.188 \pm 0.009$ & & $180.7 \pm 3.4$ & $175.1 \pm 8.4$ & $100 \pm 4.4$ \\
\hline $0630-9-42$ & 0.27 & n.d & $0.0491 \pm 0.0013$ & $0.0289 \pm 0.0016$ & $0.196 \pm 0.012$ & & $183.6 \pm 10.4$ & $181.4 \pm 11.3$ & $154 \pm 4.1$ \\
\hline $0630-9-98$ & 0.75 & n.d & $0.0514 \pm 0.0042$ & $0.0290 \pm 0.0007$ & $0.205 \pm 0.017$ & & $184.2 \pm 4.6$ & $189.7 \pm 16.1$ & $259 \pm 20.9$ \\
\hline $0630-9-10$ & 1.09 & n.d & $0.0490 \pm 0.0016$ & $0.0291 \pm 0.0010$ & $0.197 \pm 0.009$ & & $185.1 \pm 6.5$ & $182.3 \pm 8.7$ & $147 \pm 4.7$ \\
\hline 0630-9-99 & 1.40 & 0.68 & $0.0493 \pm 0.0032$ & $0.0294 \pm 0.0007$ & $0.200 \pm 0.014$ & & $186.7 \pm 4.4$ & $185.0 \pm 12.8$ & $164 \pm 10.6$ \\
\hline
\end{tabular}


Table 1. Continued

\begin{tabular}{|c|c|c|c|c|c|c|c|c|c|}
\hline $\begin{array}{l}\text { Sample } \\
\text { Code }\end{array}$ & $\mathrm{Th} / \mathrm{U}^{2}$ & $\begin{array}{c}{ }^{206} \mathrm{Pbc}^{(\mathrm{a})} \\
(\%)\end{array}$ & ${ }^{207} \mathrm{~Pb} /{ }^{206} \mathrm{~Pb}$ & ${ }^{206} \mathrm{~Pb} /{ }^{238} \mathrm{U}$ & $\begin{array}{c}{ }^{207} \mathrm{~Pb} /{ }^{235} \mathrm{U} \\
\begin{array}{c}\text { Error } \\
2 \sigma\end{array}\end{array}$ & $\begin{array}{c}\operatorname{Disc}^{(b)} \\
(\%)\end{array}$ & $\begin{array}{cc}{ }^{238} \mathrm{U}^{206} \mathrm{~Pb} & \text { Error } \\
\text { age }(\mathrm{Ma}) & 2 \sigma\end{array}$ & $\begin{array}{c}{ }^{235} \mathrm{U}_{-}{ }^{207} \mathrm{~Pb} \\
\text { age }(\mathrm{Ma})\end{array}$ & $\begin{array}{c}{ }^{207} \mathrm{~Pb}-{ }^{206} \mathrm{~Pb} \text { Error } \\
\text { age (Ma) } \quad 2 \sigma\end{array}$ \\
\hline $0630-9-25$ & 0.88 & n.d & $0.0524 \pm 0.0028$ & $0.0294 \pm 0.0005$ & $0.213 \pm 0.012$ & & $186.9 \pm 3.3$ & $195.7 \pm 11.1$ & $303 \pm 16.3$ \\
\hline $0630-9-7$ & 1.88 & n.d & $0.0540 \pm 0.0050$ & $0.0295 \pm 0.0011$ & $0.220 \pm 0.022$ & & $187.6 \pm 7.2$ & $201.7 \pm 20.3$ & $371 \pm 34.5$ \\
\hline $0630-9-37$ & 0.61 & 0.65 & $0.0490 \pm 0.0035$ & $0.0298 \pm 0.0006$ & $0.201 \pm 0.015$ & & $189.2 \pm 3.9$ & $186.1 \pm 13.8$ & $148 \pm 10.6$ \\
\hline $0630-9-35$ & 0.42 & n.d & $0.0482 \pm 0.0034$ & $0.0298 \pm 0.0006$ & $0.198 \pm 0.015$ & & $189.5 \pm 3.9$ & $183.8 \pm 13.6$ & $112 \pm 8.0$ \\
\hline $0630-9-84$ & 0.82 & n.d & $0.0487 \pm 0.0031$ & $0.0300 \pm 0.0012$ & $0.201 \pm 0.015$ & & $190.5 \pm 7.6$ & $186.3 \pm 14.0$ & $134 \pm 8.5$ \\
\hline $0630-9-88$ & 0.53 & n.d & $0.0513 \pm 0.0034$ & $0.0301 \pm 0.0006$ & $0.213 \pm 0.015$ & & $191.0 \pm 4.0$ & $195.8 \pm 13.7$ & $254 \pm 17.0$ \\
\hline $0630-9-86$ & 0.63 & n.d & $0.0566 \pm 0.0032$ & $0.0302 \pm 0.0006$ & $0.236 \pm 0.014$ & -3.1 & $192.0 \pm 3.9$ & $215.0 \pm 13.0$ & $475 \pm 27.1$ \\
\hline $0630-9-40$ & 0.70 & n.d & $0.0512 \pm 0.0044$ & $0.0302 \pm 0.0007$ & $0.213 \pm 0.019$ & & $192.0 \pm 4.2$ & $196.3 \pm 17.3$ & $249 \pm 21.2$ \\
\hline $0630-9-100$ & 1.61 & n.d & $0.0498 \pm 0.0024$ & $0.0304 \pm 0.0007$ & $0.209 \pm 0.011$ & & $193.2 \pm 4.3$ & $192.7 \pm 10.1$ & $187 \pm 8.9$ \\
\hline $0630-9-12$ & 0.66 & n.d & $0.0516 \pm 0.0024$ & $0.0304 \pm 0.0005$ & $0.217 \pm 0.010$ & & $193.3 \pm 2.9$ & $199.1 \pm 9.6$ & $269 \pm 12.3$ \\
\hline $0630-9-57$ & 0.98 & n.d & $0.0524 \pm 0.0038$ & $0.0305 \pm 0.0011$ & $0.220 \pm 0.018$ & & $193.6 \pm 7.2$ & $202.2 \pm 16.6$ & $305 \pm 22.3$ \\
\hline $0630-9-32$ & 0.56 & n.d & $0.0524 \pm 0.0049$ & $0.0305 \pm 0.0007$ & $0.220 \pm 0.021$ & & $193.7 \pm 4.4$ & $202.1 \pm 19.4$ & $302 \pm 28.1$ \\
\hline $0630-9-69$ & 0.52 & n.d & $0.0537 \pm 0.0045$ & $0.0305 \pm 0.0012$ & $0.226 \pm 0.021$ & & $193.8 \pm 7.4$ & $206.9 \pm 19.0$ & $359 \pm 30.1$ \\
\hline $0630-9-22$ & 0.47 & n.d & $0.0512 \pm 0.0037$ & $0.0306 \pm 0.0006$ & $0.216 \pm 0.016$ & & $194.1 \pm 3.7$ & $198.4 \pm 14.7$ & $251 \pm 17.9$ \\
\hline $0630-9-2$ & 0.79 & n.d & $0.0498 \pm 0.0037$ & $0.0306 \pm 0.0011$ & $0.211 \pm 0.017$ & & $194.6 \pm 7.2$ & $194.0 \pm 16.0$ & $188 \pm 13.8$ \\
\hline $0630-9-38$ & 0.56 & n.d & $0.0557 \pm 0.0046$ & $0.0307 \pm 0.0007$ & $0.236 \pm 0.020$ & & $195.2 \pm 4.3$ & $215.2 \pm 18.6$ & $440 \pm 36.7$ \\
\hline $0630-9-50$ & 1.02 & n.d & $0.0493 \pm 0.0019$ & $0.0308 \pm 0.0010$ & $0.209 \pm 0.011$ & & $195.3 \pm 6.2$ & $192.8 \pm 9.7$ & $163 \pm 6.4$ \\
\hline $0630-9-41$ & 0.53 & n.d & $0.0429 \pm 0.0037$ & $0.0308 \pm 0.0018$ & $0.183 \pm 0.019$ & & $195.8 \pm 11.4$ & $170.3 \pm 17.8$ & \\
\hline $0630-9-43$ & 0.58 & n.d & $0.0517 \pm 0.0030$ & $0.0309 \pm 0.0018$ & $0.220 \pm 0.018$ & & $196.2 \pm 11.3$ & $202.2 \pm 16.5$ & $274 \pm 15.9$ \\
\hline $0630-9-47$ & 0.78 & n.d & $0.0448 \pm 0.0030$ & $0.0309 \pm 0.0010$ & $0.191 \pm 0.014$ & & $196.2 \pm 6.5$ & $177.5 \pm 13.3$ & \\
\hline $0630-9-77$ & 0.49 & n.d & $0.0487 \pm 0.0029$ & $0.0309 \pm 0.0012$ & $0.208 \pm 0.015$ & & $196.4 \pm 7.8$ & $191.7 \pm 13.7$ & $135 \pm 8.0$ \\
\hline $0630-9-73$ & 0.47 & n.d & $0.0473 \pm 0.0027$ & $0.0310 \pm 0.0011$ & $0.202 \pm 0.014$ & & $196.6 \pm 7.0$ & $186.8 \pm 12.6$ & $65 \pm 3.7$ \\
\hline $0630-9-70$ & 0.97 & n.d & $0.0507 \pm 0.0021$ & $0.0311 \pm 0.0011$ & $0.217 \pm 0.012$ & & $197.3 \pm 6.9$ & $199.7 \pm 10.8$ & $229 \pm 9.5$ \\
\hline $0630-9-21$ & 0.71 & n.d & $0.0503 \pm 0.0036$ & $0.0312 \pm 0.0006$ & $0.216 \pm 0.016$ & & $197.9 \pm 3.8$ & $198.7 \pm 14.6$ & $208 \pm 14.7$ \\
\hline $0630-9-74$ & 1.66 & n.d & $0.0496 \pm 0.0028$ & $0.0312 \pm 0.0011$ & $0.214 \pm 0.014$ & & $198.3 \pm 7.1$ & $196.7 \pm 13.0$ & $178 \pm 9.9$ \\
\hline $0630-9-85$ & 0.79 & n.d & $0.0459 \pm 0.0033$ & $0.0313 \pm 0.0013$ & $0.198 \pm 0.016$ & & $198.5 \pm 8.0$ & $183.4 \pm 15.0$ & \\
\hline $0630-9-20$ & 0.70 & n.d & $0.0505 \pm 0.0044$ & $0.0316 \pm 0.0006$ & $0.220 \pm 0.020$ & & $200.4 \pm 3.9$ & $201.7 \pm 18.0$ & $218 \pm 19.0$ \\
\hline $0630-9-6$ & 2.11 & n.d & $0.0490 \pm 0.0030$ & $0.0316 \pm 0.0012$ & $0.214 \pm 0.015$ & & $200.6 \pm 7.3$ & $196.7 \pm 14.0$ & $151 \pm 9.2$ \\
\hline $0630-9-80$ & 0.62 & n.d & $0.0508 \pm 0.0028$ & $0.0320 \pm 0.0013$ & $0.224 \pm 0.015$ & & $202.9 \pm 8.0$ & $205.3 \pm 14.0$ & $234 \pm 13.0$ \\
\hline $0630-9-66$ & 0.61 & n.d & $0.0578 \pm 0.0037$ & $0.0320 \pm 0.0012$ & $0.255 \pm 0.019$ & -1.4 & $203.3 \pm 7.5$ & $230.8 \pm 17.1$ & $521 \pm 33.6$ \\
\hline 0630-9-68 & 0.46 & n.d & $0.0549 \pm 0.0040$ & $0.0339 \pm 0.0013$ & $0.257 \pm 0.021$ & & $214.8 \pm 8.0$ & $231.9 \pm 18.9$ & $409 \pm 29.7$ \\
\hline $0630-9-24$ & 0.39 & 0.12 & $0.0539 \pm 0.0014$ & $0.0370 \pm 0.0006$ & $0.275 \pm 0.008$ & -0.5 & $234.0 \pm 3.7$ & $246.4 \pm 7.6$ & $367 \pm 9.7$ \\
\hline $0630-9-54$ & 0.85 & n.d & $0.0556 \pm 0.0019$ & $0.0377 \pm 0.0012$ & $0.289 \pm 0.013$ & & $238.3 \pm 7.5$ & $257.6 \pm 12.0$ & $437 \pm 15.1$ \\
\hline $0630-9-58$ & 1.16 & n.d & $0.0550 \pm 0.0030$ & $0.0393 \pm 0.0014$ & $0.298 \pm 0.020$ & & $248.7 \pm 9.1$ & $265.1 \pm 17.3$ & $413 \pm 22.4$ \\
\hline $0630-9-36$ & 1.41 & n.d & $0.0514 \pm 0.0021$ & $0.0394 \pm 0.0007$ & $0.279 \pm 0.013$ & & $249.3 \pm 4.6$ & $250.1 \pm 11.4$ & $258 \pm 10.8$ \\
\hline $0630-9-65$ & 0.86 & n.d & $0.0537 \pm 0.0014$ & $0.0407 \pm 0.0014$ & $0.302 \pm 0.013$ & & $257.4 \pm 9.1$ & $267.8 \pm 11.7$ & $361 \pm 9.3$ \\
\hline $0630-9-28$ & 0.65 & n.d & $0.0611 \pm 0.0034$ & $0.0415 \pm 0.0008$ & $0.350 \pm 0.020$ & -7.6 & $262.1 \pm 4.8$ & $304.4 \pm 17.6$ & $643 \pm 35.3$ \\
\hline $0630-9-46$ & 0.83 & n.d & $0.0519 \pm 0.0031$ & $0.0421 \pm 0.0014$ & $0.301 \pm 0.020$ & & $266.0 \pm 8.7$ & $267.5 \pm 18.2$ & $282 \pm 16.8$ \\
\hline $0630-9-8$ & 0.78 & n.d & $0.0505 \pm 0.0038$ & $0.0424 \pm 0.0016$ & $0.295 \pm 0.025$ & & $267.4 \pm 10.0$ & $262.6 \pm 22.3$ & $220 \pm 16.8$ \\
\hline $0630-9-26$ & 0.73 & n.d & $0.1125 \pm 0.0019$ & $0.2778 \pm 0.0043$ & $4.311 \pm 0.099$ & -13.0 & $1580 \pm 24$ & $1695 \pm 39$ & $1841 \pm 31$ \\
\hline $0630-9-97$ & 0.99 & 1.58 & $0.1139 \pm 0.0032$ & $0.2800 \pm 0.0060$ & $4.397 \pm 0.156$ & -11.6 & $1591 \pm 34$ & $1712 \pm 61$ & $1863 \pm 53$ \\
\hline $0630-9-4$ & 0.37 & n.d & $0.1136 \pm 0.0023$ & $0.2909 \pm 0.0101$ & $4.557 \pm 0.182$ & -7.2 & $1646 \pm 57$ & $1741 \pm 70$ & $1859 \pm 37$ \\
\hline $0630-9-18$ & 0.50 & 0.06 & $0.1146 \pm 0.0021$ & $0.2936 \pm 0.0038$ & $4.637 \pm 0.103$ & -9.5 & $1659 \pm 21$ & $1756 \pm 39$ & $1873 \pm 34$ \\
\hline $0630-9-75$ & 0.19 & n.d & $0.1227 \pm 0.0028$ & $0.2976 \pm 0.0101$ & $5.033 \pm 0.206$ & -12.8 & $1679 \pm 57$ & $1825 \pm 75$ & $1996 \pm 45$ \\
\hline $0630-9-48$ & 0.48 & 0.33 & $0.1107 \pm 0.0019$ & $0.2982 \pm 0.0092$ & $4.550 \pm 0.161$ & -2.7 & $1682 \pm 52$ & $1740 \pm 62$ & $1811 \pm 31$ \\
\hline $0630-9-64$ & 0.81 & 0.02 & $0.1148 \pm 0.0021$ & $0.3005 \pm 0.0106$ & $4.756 \pm 0.189$ & -5.2 & $1694 \pm 60$ & $1777 \pm 71$ & $1877 \pm 35$ \\
\hline $0630-9-13$ & 0.57 & 0.10 & $0.1124 \pm 0.0018$ & $0.3018 \pm 0.0038$ & $4.677 \pm 0.096$ & -5.2 & $1700 \pm 21$ & $1763 \pm 36$ & $1839 \pm 30$ \\
\hline
\end{tabular}


Table 1. Continued

\begin{tabular}{|c|c|c|c|c|c|c|c|c|c|c|c|c|c|}
\hline $\begin{array}{l}\text { Sample } \\
\text { Code }\end{array}$ & $\mathrm{Th} / \mathrm{U}$ & $\begin{array}{c}{ }^{206} \mathrm{Pbc}^{(\mathrm{a})} \\
(\%)\end{array}$ & ${ }^{207} \mathrm{~Pb} /{ }^{206} \mathrm{~Pb}$ & $\begin{array}{c}\text { Error } \\
2 \sigma\end{array}$ & ${ }^{206} \mathrm{~Pb} /{ }^{238} \mathrm{U}$ & $\begin{array}{c}\text { Error } \\
2 \sigma\end{array}$ & ${ }^{207} \mathrm{~Pb} /{ }^{235} \mathrm{U} \begin{array}{c}\text { Error } \\
2 \sigma\end{array}$ & $\begin{array}{c}\operatorname{Disc}^{(b)} \\
(\%)\end{array}$ & $\begin{array}{l}{ }^{238} \mathrm{U}_{-}^{206} \mathrm{~Pb} \\
\text { age (Ma) }\end{array}$ & $\begin{array}{c}\text { Error } \\
2 \sigma\end{array}$ & $\begin{array}{l}{ }^{235} \mathrm{U}^{207} \mathrm{~Pb} \\
\text { age (Ma) }\end{array}$ & $\begin{array}{c}\text { Error } \\
2 \sigma\end{array}$ & $\begin{array}{c}{ }^{207} \mathrm{~Pb}^{-206} \mathrm{~Pb} \text { Error } \\
\text { age }(\mathrm{Ma}) \quad 2 \sigma\end{array}$ \\
\hline $0630-9-81$ & 1.04 & n.d & $0.1183 \pm 0$ & .0024 & $0.3027 \pm 0$ & 0.0115 & $4.938 \pm 0.212$ & -7.2 & $1705 \pm$ & & $1809 \pm$ & & $1931 \pm 39$ \\
\hline $0630-9-16$ & 0.01 & n.d & $0.1148 \pm 0$ & .0018 & $0.3037 \pm 0$ & 0.0038 & $4.804 \pm 0.096$ & -6.8 & $1709 \pm$ & & $1786 \pm$ & & $1876 \pm 29$ \\
\hline $0630-9-9$ & 0.09 & 0.04 & $0.1131 \pm 0$ & .0023 & $0.3065 \pm$ & 0.0106 & $4.778 \pm 0.191$ & -1.7 & $1723 \pm$ & & $1781 \pm$ & & $1850 \pm 37$ \\
\hline $0630-9-33$ & 0.62 & 0.07 & $0.1142 \pm 0$ & .0023 & $0.3099 \pm 0$ & 0.0054 & $4.880 \pm 0.129$ & -3.5 & $1740 \pm$ & & $1799 \pm$ & & $1868 \pm 37$ \\
\hline 0630-9-49 & 0.19 & 0.04 & $0.1156 \pm 0$ & .0017 & $0.3177 \pm 0$ & 0.0098 & $5.065 \pm 0.174$ & -1.6 & $1779 \pm$ & & $1830 \pm$ & & $1890 \pm 28$ \\
\hline $0630-9-53$ & 0.35 & 0.13 & $0.1128 \pm 0$ & .0015 & $0.3186 \pm 0$ & 0.0098 & $4.953 \pm 0.166$ & & $1783 \pm$ & & $1811 \pm$ & & $1845 \pm 25$ \\
\hline $0630-9-94$ & 1.08 & n.d & $0.1151 \pm 0$ & .0023 & $0.3230 \pm 0$ & 0.0057 & $5.123 \pm 0.136$ & -0.4 & $1804 \pm$ & & $1840 \pm$ & & $1881 \pm 37$ \\
\hline 0630-9-39 & 0.25 & n.d & $0.1167 \pm 0$ & .0018 & $0.3290 \pm($ & 0.0055 & $5.292 \pm 0.120$ & -0.7 & $1833 \pm$ & & $1868 \pm$ & & $1906 \pm 29$ \\
\hline $0630-9-59$ & 0.42 & n.d & $0.1151 \pm 0$ & .0020 & $0.3304 \pm 0$ & 0.0116 & $5.245 \pm 0.206$ & & $1840 \pm$ & & $1860 \pm$ & & $1883 \pm 33$ \\
\hline $0630-9-78$ & 0.42 & 0.04 & $0.1205 \pm 0$ & .0024 & $0.3327 \pm 0$ & 0.0126 & $5.530 \pm 0.237$ & -0.2 & $1851 \pm$ & & $1905 \pm$ & & $1965 \pm 39$ \\
\hline $0630-9-27$ & 0.40 & n.d & $0.1286 \pm 0$ & .0017 & $0.3355 \pm 0$ & 0.0050 & $5.946 \pm 0.118$ & -8.5 & $1865 \pm$ & & $1968 \pm$ & & $2079 \pm 27$ \\
\hline $0630-9-11$ & 0.03 & 0.11 & $0.1230 \pm 0$ & .0019 & $0.3431 \pm 0$ & 0.0042 & $5.817 \pm 0.114$ & -2.3 & $1902 \pm$ & & $1949 \pm$ & & $2000 \pm 31$ \\
\hline $0630-9-56$ & 0.51 & 0.16 & $0.1221 \pm 0$ & .0020 & $0.3522 \pm 0$ & 0.0124 & $5.930 \pm 0.231$ & & $1945 \pm$ & & $1966 \pm$ & & $1988 \pm 33$ \\
\hline $0630-9-96$ & 0.33 & n.d & $0.1374 \pm 0$ & .0032 & $0.3553 \pm 0$ & 0.0074 & $6.732 \pm 0.211$ & -7.3 & $1960 \pm$ & & $2077 \pm$ & & $2195 \pm 52$ \\
\hline $0630-9-83$ & 0.43 & 0.39 & $0.1200 \pm 0$ & .0025 & $0.3558 \pm 0$ & 0.0135 & $5.887 \pm 0.255$ & & $1962 \pm$ & & $1959 \pm$ & & $1957 \pm 41$ \\
\hline $0630-9-51$ & 0.54 & n.d & $0.1422 \pm 0$ & .0024 & $0.4208 \pm$ & 0.0131 & $8.252 \pm 0.292$ & & $2264 \pm$ & & $2259 \pm$ & & $2255 \pm 38$ \\
\hline $0630-9-67$ & 0.87 & n.d & $0.1552 \pm 0$ & .0039 & $0.4277 \pm 0$ & 0.0147 & $9.153 \pm 0.389$ & & $2295 \pm$ & & $2353 \pm$ & $=100$ & $2405 \pm 60$ \\
\hline $0630-9-29$ & 0.37 & 0.03 & $0.1597 \pm 0$ & .0020 & $0.4581 \pm 0$ & 0.0069 & $10.084 \pm 0.198$ & & $2431 \pm$ & & $2442 \pm$ & & $2453 \pm 31$ \\
\hline
\end{tabular}

(a) Percentage of ${ }^{206} \mathrm{~Pb}$ contributed by common $\mathrm{Pb}$ on the basis of ${ }^{204} \mathrm{~Pb}$. Value of common $\mathrm{Pb}$ was assumed by Stacey and Kramers (1975) model; n.d.: no detection of ${ }^{204} \mathrm{~Pb}$.

(b) Degree of discordance (\%); negative numbers and blanks show normal discordant and concordanct within $2 \sigma$ of the analytical error, respectively.

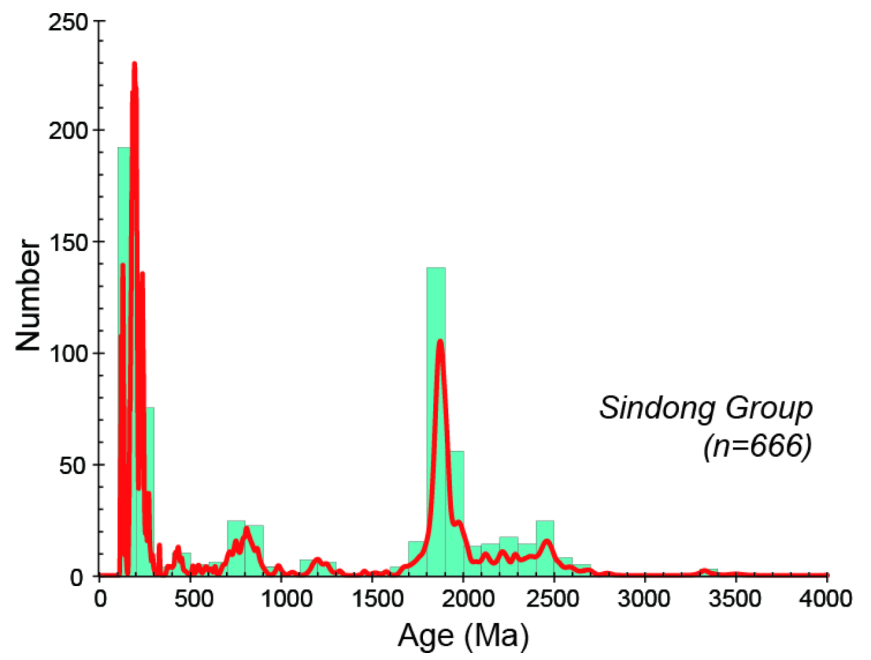

Figure 2. Relative probability plot and age histograms for detrital zircon $U-P b$ ages of the Sindong Group.

(7\%), with minor peaks at Early Paleoproterozoic and Early Neoproterozoic (Fig. 2).

\section{Nakdong Formation}

Among 300 zircons analyzed, 227 zircon grains yielded concordant or slightly discordant Paleoproterozoic to Lower Cretaceous U$\mathrm{Pb}$ ages (Fig. 3a; Table 1). Zircon $\mathrm{U}-\mathrm{Pb}$ ages of the Nakdong Formation range from $118 \pm 3 \mathrm{Ma}$ to $3494 \pm 74 \mathrm{Ma}$ and fall into four main age groups. The zircon age spectrum is dominated by Late Paleopro- terozoic ages between 1804 and $2013 \mathrm{Ma}$ (29\%) with a prominent peak at $1875 \mathrm{Ma}$, followed by Early Jurassic ages between 172 and $198 \mathrm{Ma}$ (11\%) with a peak at 183 Ma, Middle to Late Jurassic ages between 152 and $170 \mathrm{Ma}(9 \%)$ with a peak at $167 \mathrm{Ma}$, and by Late Triassic ages between 210 and $235 \mathrm{Ma}(7 \%)$ with a peak at $227 \mathrm{Ma}$. Also, there is a notable age cluster in the age range of $2307-2473 \mathrm{Ma}(8 \%)$.

Nakdong detrital zircons show spatial age variations in the basin (Fig. 3b). The age distribution patterns of the northern and central Nakdong samples are similar but different from that of the southern Nakdong sample. The latter is predominated by Paleoproterozoic and Early Jurassic detrital zircons in that order but lacks Cretaceous, Late Jurassic, Paleozoic, and Neoproterozoic zircons.

\section{Hasandong Formation}

A total of 200 zircon grains yielded concordant or slightly discordant Paleoarchean to Early Cretaceous U-Pb ages (Fig. 4a). The zircon ages are widely spread, ranging from $109 \mathrm{Ma}$ to $3360 \mathrm{Ma}$. Most zircons fall into four main age clusters: Jurassic ages between 158 and $194 \mathrm{Ma}(27 \%)$ with a peak at $177 \mathrm{Ma}$, Late Paleoproterozoic ages between 1804 and $2027 \mathrm{Ma}(22 \%)$ with a peak at $1869 \mathrm{Ma}$, Neoproterozoic ages between 731 and $844 \mathrm{Ma}(10 \%)$ with a peak at $782 \mathrm{Ma}$, and Triassic ages between 202 and $245 \mathrm{Ma}(9 \%)$ with a peak at 227 Ma. Also present is a tight Early Cretaceous age cluster between 109 and $125 \mathrm{Ma}$.

Like the underlying Nakdong Formation, the age distribution of the Hasandong detrital zircons shows spatial variations in the basin (Fig. $4 b)$. The detrital zircons of the Hasandong samples from the central and southern portions of the basin show similar age spectra, but the 

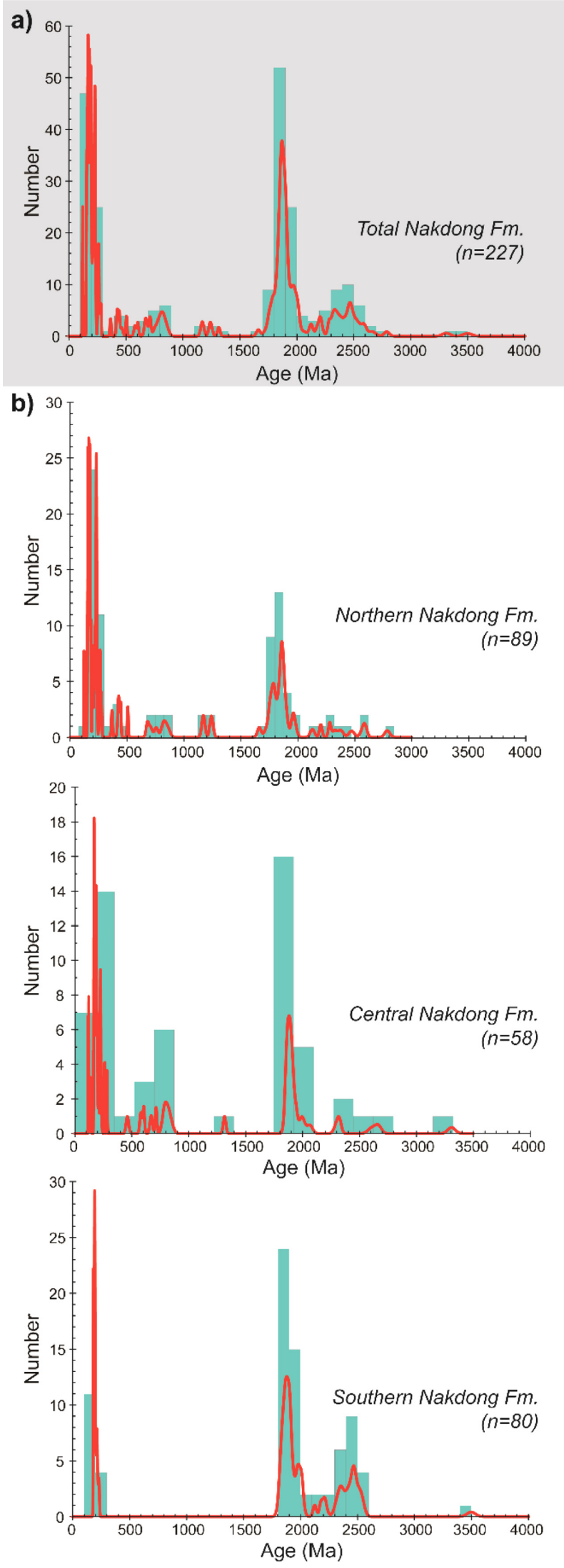

Figure 3. Relative probability plots and age histograms for (a) composite detrital zircon $U-P b$ ages of the Nakdong Formation and for (b) the Nakdong Formation in three different portions of the Nakdong Trough.
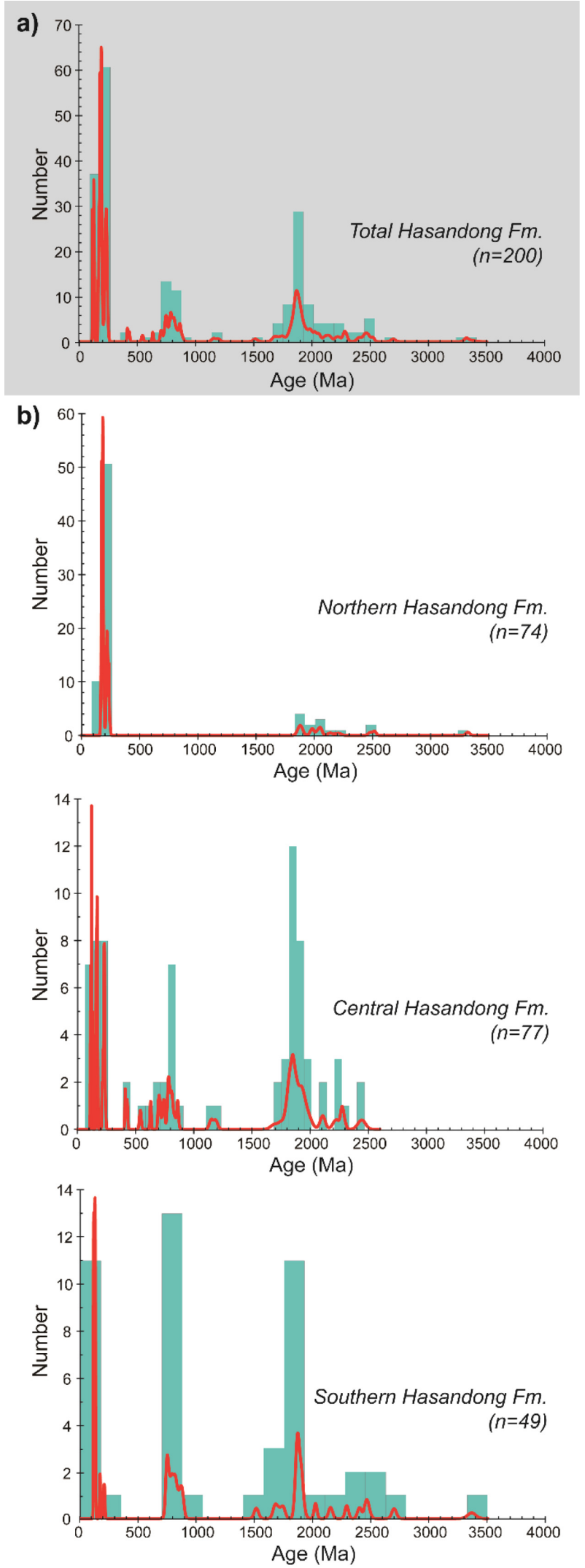

Figure 4. Relative probability plots and age histograms for (a) composite detrital zircon $U-P b$ ages of the Hasandong Formation and for (b) the Hasandong Formation in three different portions of the Nakdong Trough. 
age distribution of detrital zircons from the northern Hasandong sample is distinctive from them as evidenced by the predominance of Early Jurassic zircons followed by Triassic zircons, but lacking Neoproterozoic and Cretaceous zircons.

\section{Jinju Formation}

We obtained a total of 239 concordant or slightly discordant detrital zircon ages from the Jinju samples. The zircon ages are also widely spread, ranging from $106 \mathrm{Ma}$ to $3321 \mathrm{Ma}$ (Fig. 5a). The zircon ages fall into four major age groups: Late Paleoproterozoic ages between 1808 and $2000 \mathrm{Ma}$ (30\%) with a peak at $1968 \mathrm{Ma}$, Early Jurassic ages between 189 and $201 \mathrm{Ma}(12 \%)$ with a peak at $193 \mathrm{Ma}$, Early-Middle Jurassic ages between 163 and $188 \mathrm{Ma}(10 \%)$, and Early Cretaceous ages between 106 and $122 \mathrm{Ma}(8 \%)$ with a peak at $114 \mathrm{Ma}$. Also present are minor Early Paleoproterozoic age clusters without a prominent peak between 2064 and $2282 \mathrm{Ma}$ (7\%) and between 2318 and $2486 \mathrm{Ma}(5 \%)$.

Like the age distributions of the underlying Hasandong Formation, the age spectrum of the Jinju sample from the northern portion of the basin is separated from those of samples from the central and southern parts (Fig. 5b). Early Jurassic zircons predominate the northern Jinju sample with some Permian zircons, whereas Meso- to Neoproterozoic zircons are lacking, and Cretaceous zircons are scarcely present.

\section{Interpretation and Discussion}

As shown in Figure 2, the wide range of ages represents the great variety of Lower Cretaceous to Paleoarchean magmatic activities in the source area, indicating that Lower Cretaceous Sindong Group sediments were derived from mixed sources. The Sindong Group contains two dominant populations of $1661-2486 \mathrm{Ma}(44.8 \%)$ and $153-$ $201 \mathrm{Ma}(22.1 \%)$ detrital zircons with less abundant Triassic (9.2\%), Neoproterozoic (8.7\%), and Cretaceous (6.6\%) ages. Paleocurrent data indicate Sindong Group sediment derivation from west and northwest (Chang and Kim, 1968; Koh, 1986), most probably from the Yeongnam Massif. Thus, basement rocks and Jurassic plutons of the Yeongnam Massif likely supplied most of the 1661-2486 Ma and 153$201 \mathrm{Ma}$ zircons to the basin. In general, our detrital zircon studies adequately define the geochronology of the Yeongnam Massif.

\section{Source Rock Ages}

\section{Mesozoic Zircons}

The Mesozoic zircons in the Sindong Group range from 106-249 Ma, representing $37.8 \%$ of the analyzed zircons (Figs. 2 and 6a-c). They are first-cycle zircons produced by weathering of igneous rocks in the Yeongnam Massif as evidenced by their euhedral grain shape. The Mesozoic detrital zircons cover more extensive age range than that reported from granitic rocks in the Yeongnam Massif, 169-240 $\mathrm{Ma}$ (Cheong and Kim, 2012 and references therein) (Fig. 6e). At present, the presence of Lower Cretaceous igneous rocks is not reported from the Yeongnam Massif. Although Jwa et al. (2008) reported two age groups of Cretaceous granitic rocks in the Yeongnam Massif, 99-
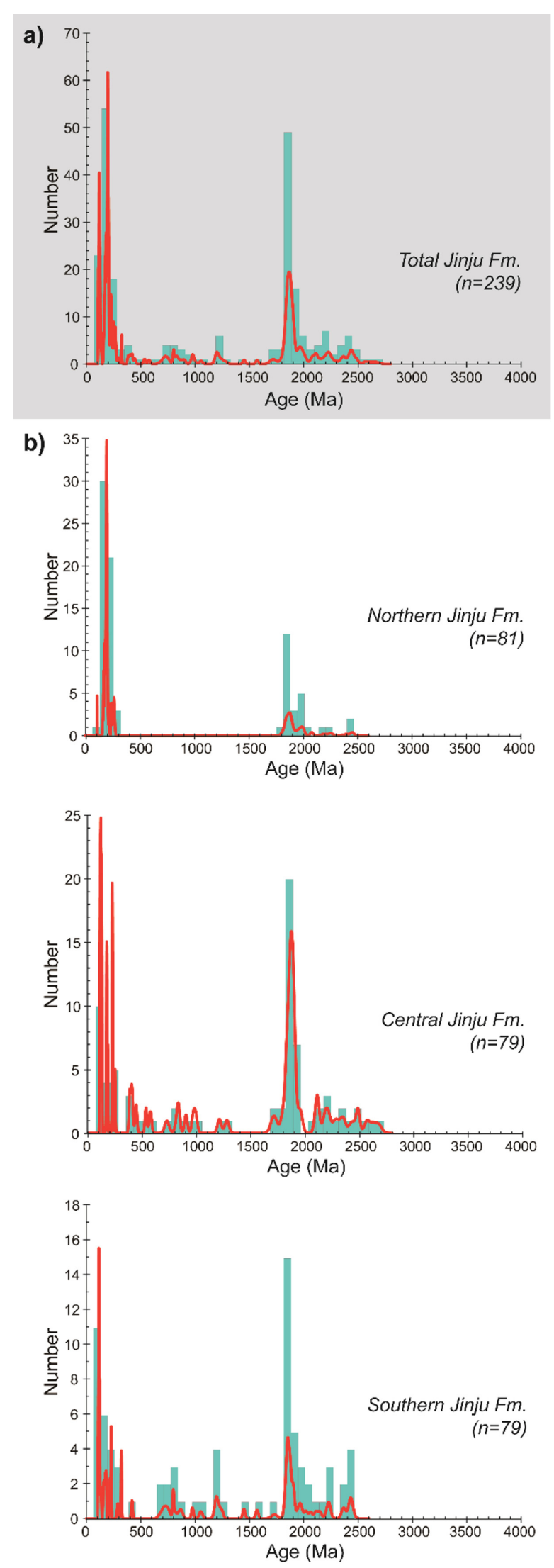

Figure 5. Relative probability plots and age histograms for (a) composite detrital zircon $U-P b$ ages of the Jinju Formation and for (b) the Jinju Formation in three different portions of the Nakdong Trough. 
(a) Cretaceous
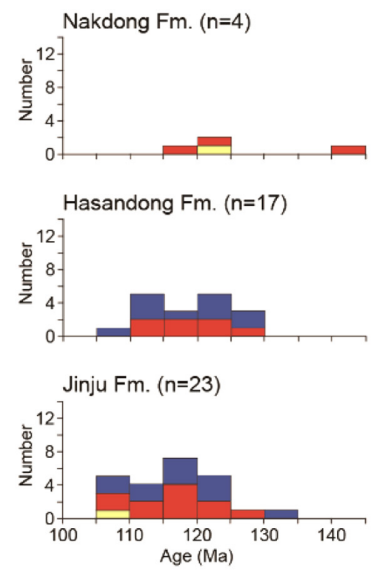

(e) Triassic - Jurassic
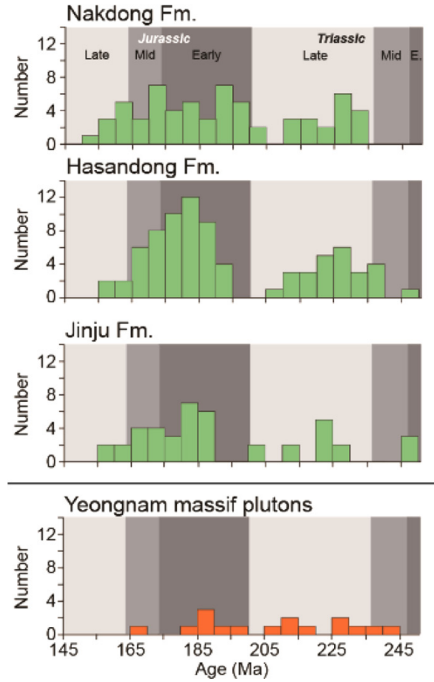

(b) Jurassic
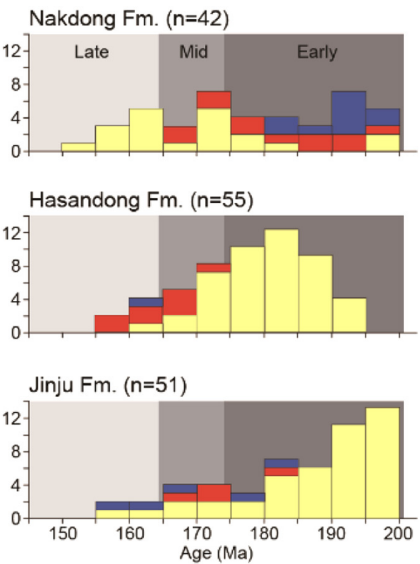

(f) Paleoproterozoic

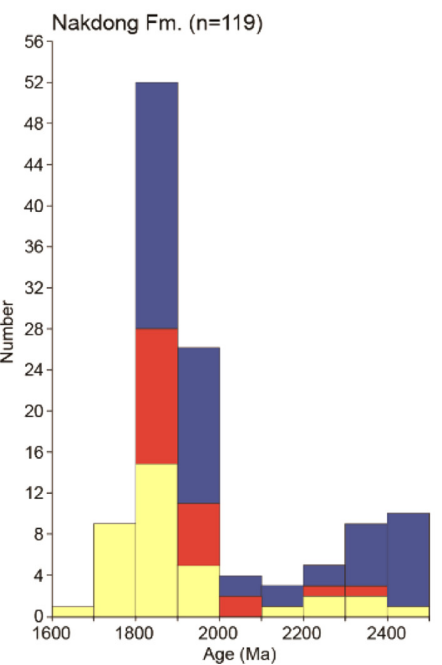

(c) Triassic
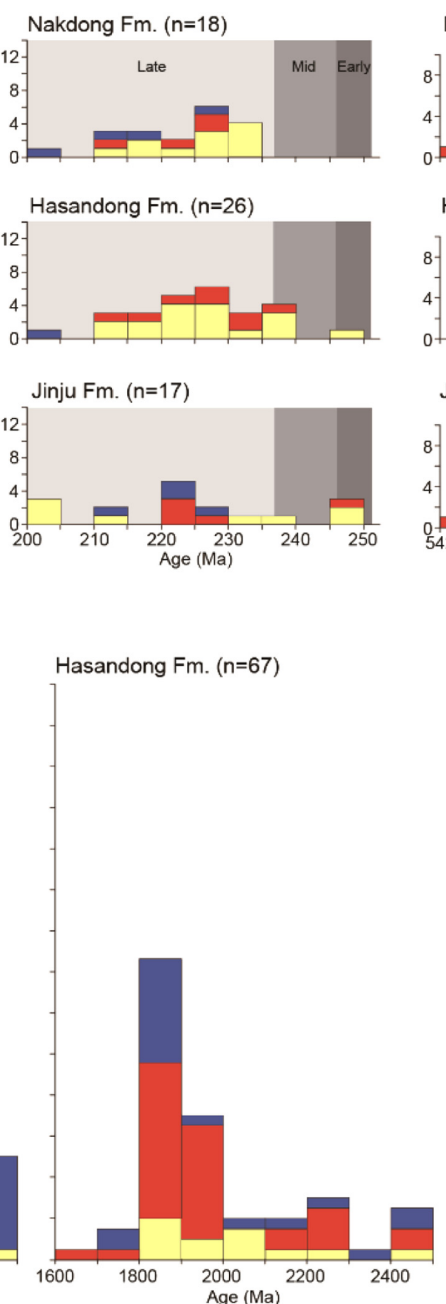

(d) Neoproterozoic

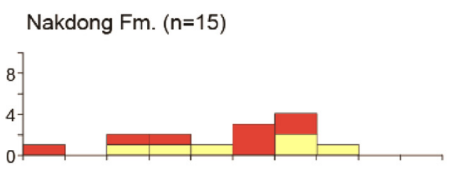

Hasandong Fm. ( $n=27)$

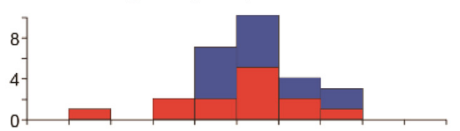

Jinju Fm. (n=15)

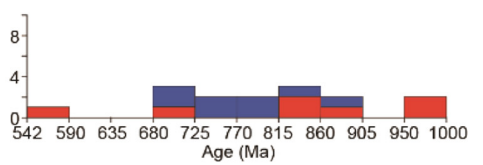

Figure 6. Age histograms of detrital zircons for (a) Cretaceous, (b) Jurassic, (c) Triassic, (d) Neoproterozoic, (e) combined Triassic-Jurassic, and (f) Paleoproterozoic in age.

$102 \mathrm{Ma}$ and $82-83 \mathrm{Ma}$, they are younger than the observed Lower Cretaceous detrital zircons in this study and the depositional age of the Sindong Group. The Okcheon fold belt to the northwest of the Yeongnam Massif also does not contain Early Cretaceous granitic rocks. The lack of reports on the presence of Lower Cretaceous igneous rocks and granitoids represented by the Sindong Group detrital zircons suggests removal of these igneous rocks in the Yeongnam Massif probably by exhumation.

Spatially, Triassic plutons were likely distributed evenly in the Yeongnam Massif during the deposition of the Sindong Group (Fig. 6c). Like the present distribution of Triassic plutons in the Yeongnam Massif and Okcheon fold belt (Fig. 1), they mostly belong to Late Triassic in age. However, during the Early Cretaceous their exposures in the Yeongnam Massif were relatively minor.

As seen in Figure 2, Jurassic zircons form the dominant contributor to the Sindong Group. Jurassic plutons were distributed heterogeneously both spatially and temporally (Fig. 6b). During the deposition of the Nakdong Formation, Lower Jurassic zircons were mainly delivered to the central and southern portions of the basin, whereas Middle to Upper Jurassic plutons were more abundantly exposed in the catchments of the northern Nakdong Trough. However, during the deposition of the Hasandong and Jinju formations, the spatial distribution of Jurassic plutons changed drastically opposite to that during the Nakdong Formation deposition. Lower Jurassic plutons with some Middle Jurassic plutons were widely distributed in the catchments of the northern Nakdong Trough, whereas, although minor, Middle to Upper Jurassic plutons existed in the drainage areas of the central and southern portions of the basin. At present, Upper Jurassic plutons are not recorded in the Yeongnam Massif (Fig. 6e) and also in the Okcheon fold belt (cf., Cheong and Kim, 2012). Kim et al. (2005) and Choi et al. (2012) interpreted that Jurassic magmatism in the southern Korean Peninsula was caused by the subduction of the Farallon-Izanagi Plate ridge. The presence of younger Jurassic detrital zircons in the Sindong Group compared to Lower Jurassic plutons in the Yeongnam Massif suggests that the younger Jurassic plutons were emplaced at shallower depths than that of the Jurassic plutons now exposed in the Yeongnam Massif. This interpretation is consistent with that of Kim et al. (2005), who reported different emplacement depths of Jurassic granitoids: the earlier stage of magmatism at deeper depths and the younger stage of magmatism at shallower depths. 
Based on hornblende geobarometric calibrations, Cho and Kwon (1994) suggested that the average emplacement depths of Jurassic granites are $12-28 \mathrm{~km}$. This indicates that uplift after Jurassic igneous activity elevated the Jurassic batholith from a depth of 12-28 km to surface, before the deposition of the Sindong Group. K-Ar biotite ages of the Middle Jurassic granitoids indicate that they were uplifted mainly between 150 and 170 Ma in South Korea (Kim et al., 2005), at an uplift rate within the range typical of orogenic areas. The rate of unroofing, or lag time, of the Yeongnam Massif was very rapid, as evidenced by enhanced exhumation of Jurassic deep plutons early in the erosional history of the Yeongnam Massif.

Based on the spatial distribution of Lower Cretaceous detrital zircons in the Nakdong Trough, Lee et al. (2010) interpreted that the magmatic activity was feeble during the Nakdong Formation deposition, the initial phase of the Nakdong Trough filling, and became progressively more active during the deposition of the Hasandong and Jinju formations. However, the locus of Lower Cretaceous magmatic activity was generally confined in the catchments of the Yeongnam Massif for the central and southern portions of the Nakdong Trough (Fig. 6a).

\section{Neoproterozoic-Mesoproterozoic Zircons}

Neoproterozoic and Mesoproterozoic detrital zircons comprise $8.7 \%$ and $2.7 \%$ of the analyzed zircons, respectively (Table 1 ). Both Neoproterozoic and Mesoproterozoic zircons show rounded to wellrounded shape, suggestive of recycling in origin. Most of the zircons of Neoproterozoic age range from 671 to $846 \mathrm{Ma}$ (Fig. 6d). In general, the source rocks for Neoproterozoic zircons were mainly exposed in the catchments of the central and northern portions of the basin. Neoproterozoic-Mesoproterozoic detrital zircons were reported from Upper Paleozoic quartzites (Park et al., 2011) and post-Ordovician metasediments (Cho et al., 2013) in the central Okcheon fold belt. However, Neoproterozoic zircons from these two sedimentary units are mostly concentrated in the early Neoproterozoic, older than 850 Ma, whereas late Mesoproterozoic ages are within the same range of Sindong Group detrital zircons. There is no known occurrence of potential zircon-bearing rocks within these age ranges in the Yeongnam Massif and also in the Okcheon fold belt, except for several rock bodies in the Okcheon fold belt formed in ca. $750 \mathrm{Ma}$ (Lee et al., 1998; Cho et al., 2004; Choi, 2013). Also, there are no known proba- ble sources that could provide Grenvillian detrital zircons in the Yeongnam Massif. Thus, further study is necessary to identify their provenance.

\section{Paleoproterozoic Zircons}

Paleoproterozoic zircons with the main peak of 1870 Ma constitute the most abundant zircons (44.8\%) in the Sindong Group sandstones (Fig. 2). Paleoproterozoic rocks are widely distributed in the Yeongnam Massif, in which 1870 Ma rocks are most abundant not only in the Yeongnam Massif but also in other Precambrian massifs in Korea (Sagong et al., 2003). Spatially, these Paleoproterozoic rocks were more widely distributed in the catchments of the Yeongnam Massif for the northern and central portions of the Nakdong Trough (Fig. 6f).

\section{Provenance Characteristics}

Based on the relative proportions of detrital zircon ages, the geology of the catchments for the Sindong Group in the Nakdong Trough was spatially different (Table 2). This interpretation is supported by Lee et al. (2015), who reported the possible existence of different drainage and fluvial system for the Nakdong Trough. The catchments for the northern portion of the Nakdong Trough apparently consisted of rocks of Jurassic $>>$ Paleoproterozoic $>$ Triassic, those for the central portion were composed of Paleoproterozoic $>$ Neoproterozoic $\sim$ Early Cretaceous $\sim$ Jurassic $>$ Triassic, and those for the southern portion were composed of Paleoproterozoic $>>$ Neoproterozoic $\sim$ Early Cretaceous $>$ Jurassic $\sim$ Mesoproterozoic. As zircon is a common constituent of felsic to intermediate igneous rocks and of sedimentary rocks (Marshall, 1967; Morton, 1985) and can survive erosion, transportation, deposition, and diagenesis, it is interpreted that the Sindong detrital zircons do not represent ages of igneous rocks in the immediate source rocks in the Yeongnam Massif. Instead, they may represent mixtures of ages from primary and ultimate source rocks. Lee et al. (2015) reported that source terranes for the Sindong Group have heterogeneous spatial and temporal distribution and were composed mainly of Precambrian basement and Triassic to Jurassic granitic rocks, with minor (meta)sedimentary rocks and syndepositional volcanic rocks. Following this information, a previous study (Lee et al., 2018a, b), and present geology of the Yeongnam Massif, Triassic and Jurassic

Table 2. Results of Kolmogorov-Smirnov (K-S) test run on the detrital zircon age data from the Sindong Group sandstones. P values larger than 0.05 are marked in yellow with bold text

\begin{tabular}{|c|c|c|c|c|c|c|c|c|c|c|}
\hline & & \multicolumn{3}{|c|}{ Nakdong Fm. } & \multicolumn{3}{|c|}{ Hasandong Fm. } & \multicolumn{3}{|c|}{ Jinju Fm. } \\
\hline & & Southern & Central & Northern & Southern & Central & Northern & Southern & Central & Northern \\
\hline \multirow{3}{*}{ Nakdong Fm. } & Southern & & 0.000 & 0.000 & 0.000 & 0.000 & 0.000 & 0.000 & 0.008 & 0.000 \\
\hline & Central & 0.000 & & 0.246 & 0.253 & 0.874 & 0.000 & 0.705 & 0.239 & 0.001 \\
\hline & Northern & 0.000 & 0.246 & & 0.063 & 0.443 & 0.000 & 0.340 & 0.033 & 0.000 \\
\hline \multirow{3}{*}{ Hasandong Fm. } & Southern & 0.000 & 0.253 & 0.063 & & 0.674 & 0.000 & 0.860 & 0.356 & 0.000 \\
\hline & Central & 0.000 & 0.874 & 0.443 & 0.674 & & 0.000 & 0.897 & 0.330 & 0.000 \\
\hline & Northern & 0.000 & 0.000 & 0.000 & 0.000 & 0.000 & & 0.000 & 0.000 & 0.146 \\
\hline \multirow{3}{*}{ Jinju Fm. } & Southern & 0.000 & 0.705 & 0.340 & 0.860 & 0.897 & 0.000 & & 0.656 & 0.000 \\
\hline & Central & 0.008 & 0.239 & 0.033 & 0.356 & 0.330 & 0.000 & 0.656 & & 0.000 \\
\hline & Northern & 0.000 & 0.001 & 0.000 & 0.000 & 0.000 & 0.146 & 0.000 & 0.000 & \\
\hline
\end{tabular}


zircons were derived from granitic rocks, Paleoproterozoic zircons from basement rocks, and Early Cretaceous zircons from the syndepositional volcanic rocks. Detrital zircons of other ages might have been derived from recycling of (meta)sedimentary rocks that were distributed in the Yeongnam Massif, whose areal extent was not significant compared to that of basement and Triassic-Lower Cretaceous igneous rocks, all of which are related to the several orogenic events (Sagong et al., 2003; Choi et al., 2012).

\section{Drainage System}

Probabilities ( $p$ values of the Kolmogorov-Smirnoff (K-S) test) $>0.05$ indicate that the confidence by which the pair of samples can be statistically distinguished is $\leq 95 \%$. Based on $p$ values, the Sindong Group samples were subdivided into three groups: the first group includes northern and central Nakdong samples and central and southern Hasandong and Jinju samples; the second group includes northern Hasandong and northern Jinju samples, and the third includes the southern Nakdong sample. Six samples of the first group show very high $p$ values of paired comparisons. The $p$ values range up to 0.897 , and average at a high value of 0.465 (Table 2 ). These very high $p$ values do not indicate statistically significant differences among the samples, and they strongly suggest that the samples were derived from a common underlying population. Although the probability of the K-S test between the Hasandong and Jinju samples in the northern portion of the basin (the second group) is relatively low ( $p=0.146$ ), the statistical analysis suggests that these two formations cannot be distinguished as being derived from separate populations (cf., Press et al., 1986).

The dissimilarity among the samples can be visualized to multidimensional scaling (MDS) map (Fig. 7; Vermeesch, 2013; Vermeesch et al., 2016). MDS is a robust superset of principal component analysis, a technique to compare multimple samples by dimension reducing (Vermeesch, 2013). It makes a map plotting the samples according to their dissimilarity: more similar ones closer, less ones farther. The MDS map of the Sindong Group sandstones analyzed in this study (Fig. 7a) and that of the compiled detrital zircon age data from the previous studies (Kim and Lim, 2017; Lee et al., 2017, 2018a, b; Fig. $7 b$ ) with this study yield consistent results with the K-S test, dividing the samples into the same three groups inspite the heterogeneity of individual samples in Figure $7 \mathrm{~b}$.

The three distinguished sample groups may represent different geology of catchments, suggesting that during the Sindong Group deposition at least three different drainage systems were developed on the footwall sources.

\section{Northern Nakdong Trough}

In the northern portion of the basin, there were at least two different drainage systems: one for the Nakdong Formation and the other for the Hasandong and Jinju formations. This interpretation may be supported by the sedimentological work by Rhee et al. (1988) and Jo and Chough (2001), who studied the Sindong Group in the northwestern corner of the Gyeongsang Basin. They interpreted that the Sindong Group was deposited eastward following the eastward shift in the locus of the sediment deposition without changes in sediment dispersal pattern to south- to southeastward due to tilting or block faulting of the basin floor along the Andong Fault, the northern boundary of the Nakdong Trough, under extensional or transtensional tectonic regime. If this paleogeographic reconstruction of the Nakdong Trough is accepted, the paleofluvial system draining into the northern Nakdong Trough during the Nakdong Formation deposition could not catch up the migrating depocenters to the right and the new paleofluvial system was developed from the north during the deposition of the Hasandong and Jinju formations. This interpretation may partly explain the relatively low $p$ values between the Hasandong and Jinju samples in the northern portion of the basin. After the deposition of
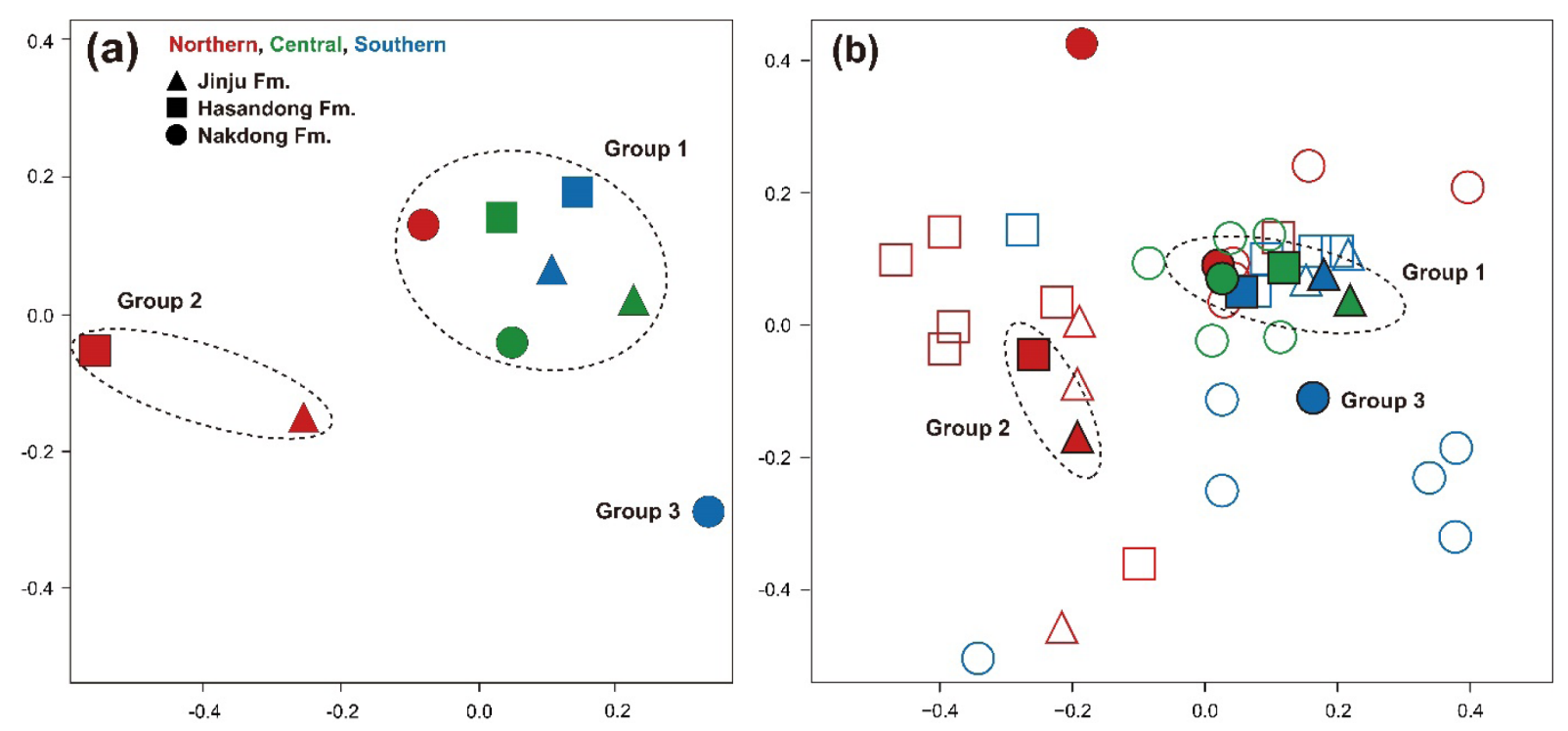

Figure 7. MDS map of (a) the detrital zircon ages of the Sindong Group sandstones in this study and (b) compiled zircon age data from this study and previous studies (Kim and Lim, 2017; Lee et al., 2017; Lee et al., 2018a,b). The more similar samples are located close together, whereas the less same samples farther, making three sample groups identical to those of the K-S test. The solid circles in (b) are the integrated data from the each part of the formation. Note that these integrated data show the same three groups inspite heterogeneity of the data from the individual samples. 
the Hasandong Formation, the successive eastward development of the depocenter might have caused adjustment of the paleofluvial system by incorporating slightly different mix of source rocks to the newly formed depocenter for the Jinju Formation deposition. However, the studied section in this study is far south to southeast of the Rhee et al. (1988)'s study area, and thus this scenario may not hold true for this study. Rather, it is interpreted that the Nakdong paleoflow system kept its course through the deposition of the Hasandong and Jinju formations and that the drainage basin became sourced from topographic highs composed more of Jurassic granitoids than that for the Nakdong Formation.

The change in provenance between the Nakdong and Hasandong formations is supported by the nearly two-fold increase in feldspar content among the framework grains (Lee et al., 2015). This was likely caused by strong influences resulting from confluence of dispersal paths draining Jurassic granitoids. In other words, provenance signatures of the paleoflow systems were diluted downstream, as new Jurassic populations are introduced and the system attains second-order. The significantly increasing population of Jurassic age during the Hasandong Formation deposition indicates addition of or expanding Jurassic plutons in its provenance during that time frame. Lee et al. (2015) interpreted that repeated faulting activities occurred in the Yeongnam Massif at the onset of the deposition of each of the Hasandong and Jinju formations in the northern portion, which suggests readjustment of drainage areas during the deposition of the Hasandong and Jinju formations. Alternatively, outcrops of Jurassic granitoids were uncovered by downcutting of the river.

\section{Central Nakdong Trough}

The paleofluvial system for the central portion of the basin was different from that for the northern portion of the basin and kept supplying similar detritus continuously during the basin filling. This interpretation suggests that the geology of the drainage basin area did not change temporally. As the $p$ value (0.246) of the Nakdong Formation samples between the northern and central portions of the basin indicates that detrital zircons for both areas were derived from the same underlying population, which suggests that the paleofluvial system for the Nakdong Formation in both areas originated either from common topographic highs or separately in parallel from the mountain ranges composed of similar geology.

\section{Southern Nakdong Trough}

For the southern portion of the basin, two different paleofluvial systems are inferred from the K-S test for samples between the Nakdong and Hasandong-Jinju formations. The paleoflow system for the Nakdong Formation drained source terranes composed mainly of Paleoproterozoic rocks with some Jurassic rocks. This interpretation is supported by the present geology of the southwestern Yeongnam Massif, consisting of widespread Paleoproterozoic basement rocks and Jurassic granitoids with sparsely distributed Triassic granitoids (Fig. 1). On the contrary, the paleoflow system for the Hasandong and Jinju formations drained source terranes composed of rocks of mixed Paleoproterozoic, Mesoproterozoic, Neoproterozoic, and Early Cretaceous in age. Lee et al. (2015) reported that Nakdong sandstones are rich in quartz (av. 73\% of framework grains) and metamorphic rock fragments, whereas Hasandong and Jinju sandstones are rich in feldspar and volcanic rock fragments. They also reported the sudden increase in plutonic quartz grain content at the onset of the Hasandong Formation deposition. Since we do not have data from the samples between the studied samples of the Nakdong and Hasandong formations, it is yet clear if the paleofluvial system was the same during the Sindong Group deposition but only with a change in provenance characteristics with time.

\section{Hypotheses for Changes in Drainage Systems of the Nak- dong Trough}

Four hypotheses can be raised to explain the age separation of detrital zircons between the Nakdong and Hasandong-Jinju formations and similarity of age spectra of the Hasandong and Jinju formations to those of the Sindong Group in the central portion. Spatially, the fault throw was different along the western boundary fault of the Nakdong Trough. There was a large fault throw in the southern segment of the boundary fault as evidenced by the presence of relatively thick $(\sim 160$ $\mathrm{m})$ gray massive conglomerates in the lower part of the Nakdong Formation (Choi, 1981). As a river continues to drain across the large fault scarps, sediment input from footwall catchments resulting from the incision across the footwall uplifts (e.g., Cowie et al., 2006) would be increased and might dominate the sediment composition.

Considering the common tectonic control for changing drainage patterns (e.g., Hoon et al., 1995) and the extensional origin of basin formation, however, we hypothesize that the fault movements that occurred prior to or syndepositional with the deposition of the Hasandong Formation (Lee et al., 2015) likely explain the drastic changes in detrital zircon age distribution from that of the Nakdong Formation and abrupt increase in plutonic quartz grains at the onset of Hasandong Formation deposition (Lee et al., 2015). The drainage basin seemed to have widened to the source terrane having similar geology to or shared the source terrane for the paleoflow system for the central portion. Alternatively, as relay ramps between fault segments along the basin edge are commonly the entry points of river system into the hanging wall depocenters (Cowie et al., 2006), the paleoriver that supplied detritus to the Nakdong Formation might have drained low gradient hinterland catchments. Sediment supplied during the early rift-initiation stage is well-processed by the low-gradient rivers, resulting in relatively mature composition (Cowie et al., 2006), which may explain the relatively quartz-rich nature of the Nakdong sandstone. Following the faulting and linkage of the fault segments, the paleoriver might have maintained its course during the deposition of the Hasandong and Jinju formations. As a river continues to drain across the active fault scarps, sediment input from footwall catchments resulting from the incision across growing footwall uplifts (Cowie et al., 2006) would be increased and might dominate the sediment composition. In such a case, the ages of detrital zircons of the Hasandong and Jinju formations samples may represent those of footwall rocks. However, this interpretation is less likely considering the presence of considerable amount of lithic fragment in the Nakdong Formation (av. 13\% of the framework grains; Lee et al., 2015) and the statistically indistinguishable detrital zircon age spectra of the samples in the central and southern portions as shown by the $\mathrm{K}-\mathrm{S}$ 
test: $p=0.253$ for the Nakdong Formation in the central portion and the Hasandong Formation in the southern portion and $p=0.674$ for the Hasandong Formation in both portions.

A third hypothesis is that during the Hasandong Formation deposition in the southern portion, the paleoflow system developed in the central portion might have deflected toward the southern portion due to increased fault throw and slip rate that occurred at the onset of the Hasandong Formation. This interpretation is partly supported by the occurrence of relatively thick, amalgamated sandbodies ( $\sim 6 \mathrm{~m}$ thick) in the middle of the Hasandong sequence, which was interpreted to be braided fluvial deposits formed in axial channels flowing south (cf., Egawa and Lee, 2009). A similar faulting activities were suggested at the onset of the Jinju Formation deposition in the central portion by Lee et al. (2015), which suggests that following the above hypothesis the paleoflow system might have deflected back to the central portion and deposited the Jinju Formation there. However, this hypothesis does not explain the dominant sediment of the Hasandong Formation being a deposit of highly sinuous fluvial system (Choi, 1986b), the high $p$ value between the Hasandong and Jinju formations in the southern portion and between the Jinju formations in the central and southern portions ( 0.860 and 0.656 , respectively), and the paleocurrent data for the Jinju Formation in the central portion (Chang and Kim, 1968).

A fourth hypothesis is that after deposition of the Nakdong Formation, the Hasandong and Jinju formations were deposited from the paleoflow system that was bifurcated from the paleofluvial system responsible for the central portion. River bifurcation is a common phenomenon when a stream nears a lake or the ocean forming a distributary channel (channels). In this case, the river bifurcation should have been in the upper reaches of the main paleoflow system of the central portion to deposit thick Hasandong fluvial plain sediments. However, river bifurcation in the upstream is rather uncommon and

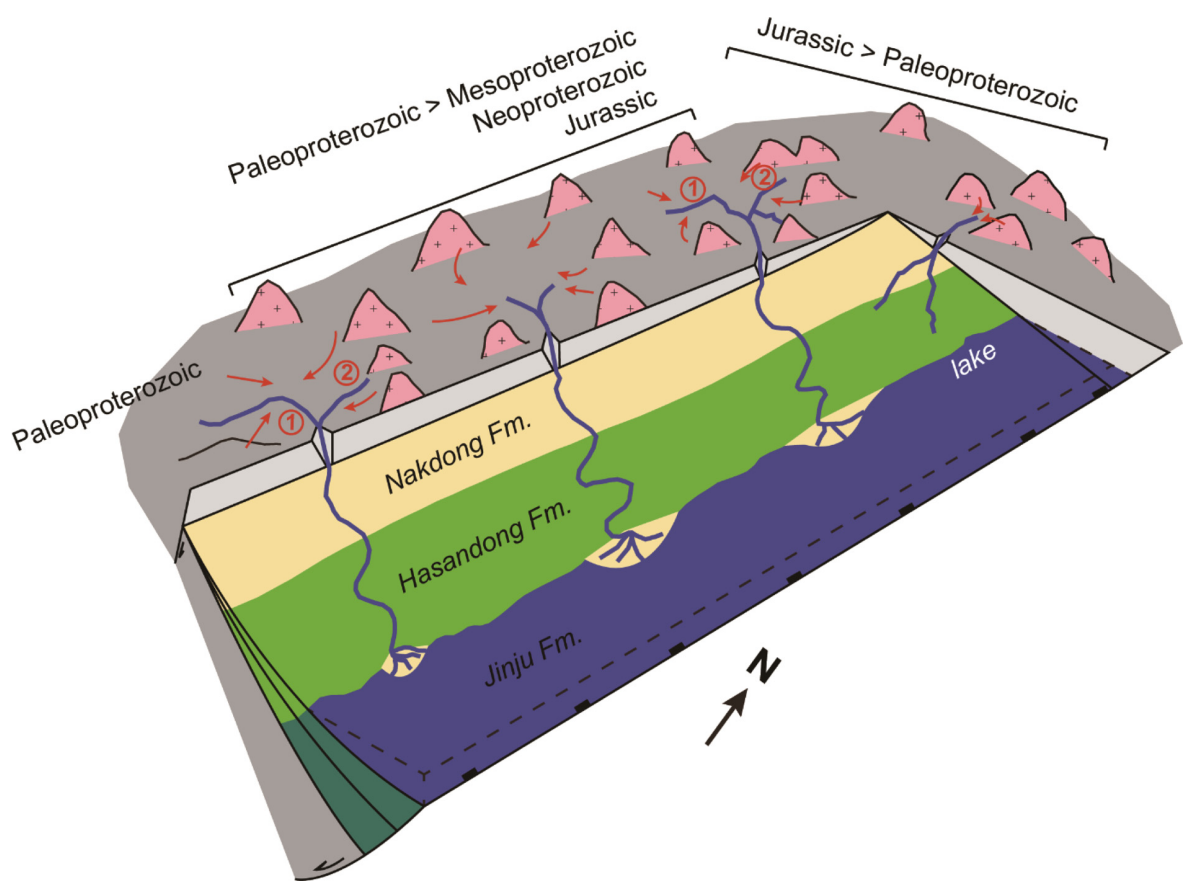

Figure 8. Schematic diagram illustrating the development of paleodrainage systems draining into the Nakdong Trough during the Early Cretaceous. The circled numbers in the northern and southern portions of the Yeongnam Massif represent the temporal changes of drainage systems: 1 for the Nakdong Formation and 2 for the Hasandong and Jinju formations. compared with the main channel, the suggested distributary channels may not have carried enough sediments to deposit thick Hasandong and Jinju formations in the southern portion.

The schematic diagram depicting the paleodrainage systems for the Sindong Group is shown in Fig. 8. The studied three portions of the basin are envisaged to have received the sediments through respective drainage-basin outlets, whose spatial spacing often tends to be regular on fault blocks (Talling et al., 1997). As the orientation of the volcanic front parallels the strike of the subducting plate, Jurassic granitoids in the Yeongnam Massif are distributed in general with a NE-SW-trending trend (Choi et al., 2012). The Yeongnam Massif to the west of the central portion is composed of complex masses of Triassic and Jurassic granitoids, which may have provided resistant substrate forming ridge crest dividing the drainage basins between the central and southern portions. The same is true for the part of the Yeongnam Massif to the west of the northern portion of the basin. Sediments to the Nakdong Trough were supplied through the orogennormal oriented valley system from at least three orogen-parallel drainage basins developed in the Yeongnam Massif. Thus, it is inferred that the geology of the Yeongnam Massif was not uniform like the present day: the central Yeongnam Massif was composed of Paleoproterozoic basement rocks and Triassic/Jurassic granitoids with Mesoproterozoic-Neoproterozoic supracrustal rocks, whereas the southwestern Yeongnam Massif was composed mostly of Paleoproterozoic basement rocks with some Jurassic granitoids.

\section{Conclusions}

To characterize the sedimentary provenance and paleodrainage system for the Sindong Group, sandstones from three different parts of the Nakdong Trough (northern, central, and southern parts) were collected for detrital zircon $\mathrm{U}-\mathrm{Pb}$ dating using a LA-ICP-MS technique. Detrital zircons show a wide range of ages from 106 Ma to $3494 \mathrm{Ma}$ with spatial and temporal variations of age population. Based on the $\mathrm{K}-\mathrm{S}$ statistical test and MDS map, zircon-age populations can be grouped into three sample groups representing respective provenance during the Nakdong Trough filling: (1) northern and central Nakdong sandstones, central and southern Hasandong sandstones, and central and southern Jinju sandstones, (2) northern Hasandong and northern Jinju sandstones, and (3) a southern Nakdong sandstone. The source terranes for the central portion of the basin were composed dominantly of Paleoproterozoic rocks with subordinate Neoproterozoic, Triassic, Jurassic, and Lower Cretaceous rocks. Sandstones from the Nakdong Formation in the northern portion and the Hasandong and Jinju formations in the southern portion of the basin were also derived from the same provenance, but through different paleodrainage system. By the time of the Hasandong For- 
mation deposition in the northern portion of the basin, the paleodrainage had switched to source terranes composed mainly of Jurassic granitoids with minor Paleoproterozoic rocks and continued supplying detritus to the overlying Jinju Formation. The paleodrainage system for the Nakdong Formation in the southern portion of the basin had proximal sources composed mainly of Paleoproterozoic rocks, but for the Hasandong and Jinju formations the paleodrainage was different from that for the underlying Nakdong Formation and was connecting pluton-parallel to the source terranes for the central portion of the basin after faulting activities at the onset of the Hasandong Formation deposition. Combined with petrographic data of Lee et al. (2015), the spatial and temporal age distribution of detrital zircons of the Sindong Group suggests evolution of paleodrainage patterns in the Yeongnam Massif in association with faulting activities during the deposition of the Sindong Group. Our model posits that at least three Early Cretaceous flows across the Yeongnam Massif funneled into the three different parts of the Nakdong Trough. This interpretation is supported by the apparent belt-like distribution of the stratigraphic units in parallel to each other along the axis of the Nakdong Trough.

\section{Acknowledgments}

This study was funded by the National Research Foundation of Korea (NRF-2014R1A2A2A01005404, 2017R1A2B4007877, 2017R1C1B1007653). The authors would like to thank H. Lee, S.K. Hong, I. Seo, and M.K. Lee for providing field and laboratory assistance.

\section{References}

Barth, A.P., Wooden, J.L., Jacobson, C.E., and Probst, K., 2004, U-Pb geochronology and geochemistry of the McCoy Mountains, southeastern California: A Cretaceous retroarc foreland basin. Geological Society of America Bulletin, v. 116, pp. 142-153.

Chang, K.H., 1970, Geology of upper Mesozoic strata, N. Gyeongsang Province, southern Korea (I). Journal of the Geological Society of Korea, v. 6, pp. 1-12.

Chang, K.H., 1975, Cretaceous stratigraphy of Southeast Korea. Journal of the Geological Society of Korea, v. 11, pp. 1-23.

Chang, K.H., 1987, Cretaceous Strata, In: Lee, D.S., (Ed.), Geology of Korea, Kyohak-Sa, Seoul, pp. 175-201.

Chang, K.H., and Kim, H.M., 1968, Cretaceous paleocurrent and sedimentation in northwestern part of Kyongsang basin, southern Korea. Journal of the Geological Society of Korea, v. 4, pp. 77-97.

Cheong, C.S., and Chang, H.W., 1996a, Geochemistry of the Daebo granite batholith in the central Ogcheon belt, Korea: a preliminary report. Economic and Environmental Geology, v. 29, pp. 483-493.

Cheong, C.S., and Chang, H.W., 1996b, Tectono-magmatism, -metamorphism, and -mineralization of the central Ogcheon belt, Korea (1). Journal of the Geological Society of Korea, v. 32, pp. 91-116.

Cheong, C.S., and Kim, N., 2012, Review of radiometric ages for Phanerozoic granitoids in southern Korean Peninsula. Journal of the Petrological Society of Korea, v. 21, pp. 173-192.

Cho, D.L., and Kwon, S.T., 1994, Hornblende geobarometry of the Mesozoic granitoids in South Korea and the evolution of crustal thickness. Journal of the Geological Society of Korea, v. 30, pp. 41-61.

Cho, M., and Kim, H., 2005, Metamorphic evolution of the Ogcheon belt, Korea: a review and new age constraints. International Geology
Review, v. 47, pp. 41-57.

Cho, M., Cheong, W., Ernst, W.G., Yi, K., and Kim, J., 2013, SHRIMP U$\mathrm{Pb}$ ages of detrital zircons in metasedimentary rocks of the central Ogcheon fold-thrust belt, Korea: Evidence for tectonic assembly of Paleozoic sedimentary protoliths. Journal of Asian Earth Sciences, v. 63, pp. 234-249.

Cho, M., Kim, T., and Kim, H., 2004, SHRIMP U-Pb zircon age of a felsic meta-tuff in the Ogcheon Metamorphic Belt, Korea: Neoproterozoic (ca. $750 \mathrm{Ma}$ ) volcanism. Journal of the Petrological Society of Korea, v. 13 , pp. 119-125.

Choi, D.K., 1985, Spores and pollen from the Gyeongsang Supergroup, southeastern Korea and their chronologic and paleoecologic implications. Journal of Paleontological Society of Korea, v. 1, pp. 33-50.

Choi, D.K., and Park, J.B., 1987, Palynology of the Jinju Formation (Lower Cretaceous), Waegwan-Daegu and Jinju areas, Korea. Journal of Paleontological Society of Korea, v. 3, pp. 28-43.

Choi, H.I., 1981, Depositional environments of the Sindong Group in the southwestern part of the Kyeongsang Basin. Ph.D. thesis, Department of Geology, Seoul National University, 144 p.

Choi, H.I., 1986, Sandstone petrology of the Sindong Group, southwestern part of the Gyeongsang Basin. Journal of the Geological Society of Korea, v. 22, pp. 212-223.

Choi, J.E., 2013, SHRIMP U-Pb age of Seochangri Formation and Geumsusan Formation in Okcheon belt. MSc. thesis, Department of Earth Science Education, Pukyoung National University, 58p.

Choi, S.J., 1987, Study on the Lower Cretaceous charophytes from the upper Gyeongsang Supergroup. Journal of Paleontological Society of Korea, v. 3, pp. 79-92.

Choi, S.J., 1989, Fossil charophytes from the Nagdong Formation in Seonsangun, Gyeongsangbukdo, Korea. Journal of Paleontological Society of Korea, v. 5, pp. 28-38.

Choi, T., and Lee, Y.I., 2011, Thermal histories of Cretaceous basins in Korea: Implications for response of the East Asian continental margin to subduction of the Paleo-Pacific Plate. Island Arc, v. 20, pp. 371-385.

Choi, T., Lee, Y.I., and Orihashi, Y., 2012, Mesozoic detrital zircon U-Pb ages of modern river sediments in Korea: implications for migration of arc magmatism in the Mesozoic East Asian continental margin. Terra Nova, v. 24, pp. 156-165.

Cowie, P.A., Attal, M., Tucker, G.E., Whittaker, A.C., Naylor, M., Ganas, A., and Roberts, G.P., 2006, Investigating the surface process response to fault interaction and linkage using a numerical modelling approach. Basin Research, v. 18, pp. 231-266.

Dickinson, W.R., and Gehrels, G.E., 2003, U-Pb ages of detrital zircons from Permian and Jurassic eolian sandstones of the Colorado Plateau, USA: Paleogeographic implications. Sedimentary Geology, v. 163, pp. 29-66.

Egawa, K., and Lee, Y.I., 2009, Jurassic synorogenic basin filling in western Korea: sedimentary response to inception of the western CircumPacific orogeny. Basin Research, v. 21, pp. 407-431.

Gehrels, G.E., and Stewart, J.H., 1998, Detrital zircon U-Pb geochronology of Cambrian to Triassic miogeoclinal and eugeoclinal strata of Sonora, Mexico. Journal of Geophysical Research, v. 103, pp. 24712487.

Hong, S.S., 2001, Implication for the emplacement depth of the granites in the Yeongnam Massif, using the aluminum-in-hornblende barometry. Journal of Petrological Society of Korea, v. 10, pp. 36-55.

Hoon, C., Guerrero, J., Sarmiento, g.A., and Lorente, M.A., 1995, Andean tectonics as a cause for changing drainage patterns in Miocene northern South America. Geology, v. 23, pp. 237-240.

Jo, H.R., and Chough, S.K., 2001, Architectural analysis of fluvial sequences in the northwestern part of Kyongsang Basin (Early Cretaceous), SE Korea. Sedimentary Geology, v. 144, pp. 307-334.

Jwa, Y.-J., 2004, Possible source rocks of Mesozoic granites in South Korea: implications for crustal evolution in NE Asia. Transactions of the Royal Society of Edinburgh: Earth Sciences, v. 95, pp. 181-198. 
Jwa, Y.J., Orihashi, Y., Kim, H.G., and Sung, K.H., Zircon U-Pb ages of the Mesozoic granites in the central Yeongnam massif: Including newly found Cretaceous granites, Proceedings Joint Geological Sciences Meeting, 2008, The Geological Society of Korea, Abstracts No.2-1, p. 14.

Kim, C.B. and Lim, H.S., 2017, Absolute dating of fossil localities and rocks designated as natural monument of Korea II, National Research Institute of Cultural Herirage, $192 \mathrm{p}$.

Kim, S.W., Oh, C.W., Choi, S.G., Ryu, I.C., and Itaya, T., 2005, Ridge subduction-related Jurassic plutonism in and around the Okcheon Metamorphic Belt, South Korea, and implications for Northeast Asian tectonics. International Geology Review, v. 47, pp. 248-269.

Koh, I.S., 1974, Sedimentary petrology of Nagdong Group (1). Journal of the Geological Society of Korea, v. 10, pp. 207-224.

Koh, I.S., 1986, Study on the source rocks of the Nagdong Group. Journal of the Geological Society of Korea, v. 22, no. 3, pp. 233-256.

Lawton, T.F., Bradford, I.A., Vega, F.J., Gehrels, G.E., and Amato, J.M., 2009, Provenance of Upper Cretaceous-Paleogene sandstones in the foreland basin system of the Sierra Madre Oriental, northeastern Mexico, and its bearing on fluvial systems of the Mexican Laramide Province. Geological Society of America Bulletin, v. 121, pp. 820-836.

Lee, C., Ryu, I.-C., and Shinn, Y.J., 2017, U-Pb ages of detrital zircons in Lower Cretaceous non-marine successions of the Gyeongsang Basin, Northeast Asia: Implications for sediment provenance. Sedimentary Geology, v. 353, pp. 125-138.

Lee, J.I., and Lee, Y.I., 2000, Provenance of the Lower Cretaceous Hayang Group, Gyeongsang Basin, southeastern Korea: Implications for continental-arc volcanism. Journal of Sedimentary Research, v. 70, pp. 151158 .

Lee, K.S., Chang, H.W., and Park, K.H., 1998, Neoproterozoic bimodal volcanism in the central Ogcheon belt, Korea: age and tectonic implication. Precambrian Research, v. 89, pp. 47-57.

Lee, S.M., Kim, H.S., and Oh, I.S., 1986, Metamorphic petrology of Precambrian gneisses in Samcheok-Jukbyeon area. Journal of the Geological Society of Korea, v. 22, pp. 257-277.

Lee, T.-H., Park, K.-H., and Yi, K., 2018a, Nature and evolution of the Cretaceous basins in the eastern margin of Eurasia: A case study of the Gyeongsang Basin, SE Korea. Journal of Asian Earth Sciences, v. 166, pp. 19-31.

Lee, T.-H., Park, K.-H., and Yi, K., 2018b, SHRIMP U-Pb ages of detrital zircons from the Early Cretaceous Nakdong Formation, South East Korea: Timing of initiation of the Gyeongsang Basin and its provenance. Island Arc, v. e12258.

Lee, Y.I., 1999, Stable isotopic composition of calcic paleosols of the Early Cretaceous Hasandong Formation, southeastern Korea. Palaeogeography, Palaeoclimatology, Palaeoecology, v. 150, pp. 123-133.

Lee, Y.I., Choi, T., Lim, H.S., and Orihashi, Y., 2010, Detrital zircon geochronology of the Cretaceous Sindong Group, SE Korea: Implications for depositional age and Early Cretaceous igneous activity. Island Arc, v. 19, pp. 647-658.

Lee, Y.I., and Lim, D.H., 2008, Sandstone diagenesis of the Lower Creta- ceous Sindong Group, Gyeongsang Basin, southeastern Korea: Implcations for compositional and paleoenvironmental controls. Island Arc, v. 17, pp. 152-171.

Lee, Y.I., Yi, J., and Choi, T., 2015, Provenance analysis of lower Cretaceous Sindong Group sandstones in the Gyeongsang Basin, Korea, using intergrated petrography, quartz SEM-cathodoluminescence, and zircon $\mathrm{Zr} / \mathrm{Hf}$ analysis. Journal of Sedimentary Research, v. 85, pp. 529-543.

Lim, H.S., Lee, Y.I., and Min, K.D., 2003, Thermal history of the Cretaceous Sindong Group, Gyeongsang Basin, Korea based on fission track analysis. Basin Research, v. 15, pp. 139-152.

Link, P.K., Fanning, C.M., and Beranek, L.P., 2005, Reliability and longitudinal change of detrital-zircon age spectra in the Snake River system, Idaho and Wyoming: An example of reproducing the bumpy barcode. Sedimentary Geology, v. 182, pp. 101-142.

Ludwig, K.R., 2003, User's manual for Isoplot 3.00. Berkeley Geochronology Center Special Publication, v. 4, pp. 1-71.

Noh, J.H., and Park, H.S., 1990, Mineral diagenesis of sandstones from the Kyonsang Supergroup in Goryeong area. Journal of the Geological Society of Korea, v. 26, pp. 371-392.

Rainbird, R.H., McNicoll, V.J., Thériault, R.J., Heaman, L.M., Abbott, J.G., Long, D.G.F., and Thorkelson, D.J., 1997, Pan-continental river system draining Grenville orogeny recorded by $\mathrm{U}-\mathrm{Pb}$ and $\mathrm{Sm}-\mathrm{Nd}$ geochronology of Neoproterozoic quartzarenites and mudrocks, northwestern Canada. The Journal of Geology, v. 105, pp. 1-17.

Raines, M.K., Hubbard, S.M., Kukulski, R.B., Leier, A.L., and Gehrels, G.E., 2013, Sedimentary dispersal in an evolving foreland: Detrital zircon geochronology from Upper Jurassic to lowermost Cretaceous strata, Alberta Basin, Canada. Geological Society of America Bulletin, v. 125, pp. 741-755.

Rhee, C.W., Jo, H.R., and Chough, S.K., 1988, An allostratigrpahic approach to a non-marine basin: the north-western part of Cretaceous Kyongsang Basin, SE Korea. Sedimentology, v. 45, pp. 449-472.

Roberts, E.M., Stevens, N.J., O'Connor, P.M., Dirks, P H.G.M., Gottfried, M.D., Clyde, W.C., Armstrong, R.A., Kemp, A.I.S., and Hemming, S., 2012, Initiation of the western branch of the East African Rift coeval with the eastern branch. Nature Geoscience, v. 5, pp. 289-294.

Seo, S.J., 1985, Lower Cretaceous geology and paleontology (Charophyta) of central Kyongsang Basin, Korea. Ph.D. Thesis, Department of Geology, Kyungpook National University, 177 p.

Shin, S.C., and Jin, M.S., 1995, Isotope age map of plutonic rocks in Korea. Korea Institute of Geology, Mining and Materials.

Talling, P.J., Stewart, m.D., Stark, C.P., Gupta, S., and Vincent, S.J., 1997, Regular spacing of drainage outlets from linear fault blocks. Basin Research, v. 9, pp. 275-302.

Vermeesch, P., 2013, Multi-sample comparison of detrital age distributions. Chemical Geology, v. 341, pp. 140-146.

Vermeesch, P., Resentini, A., and Garzanti, E., 2016, An R package for statistical provenance analysis. Sedimentary Geology, v. 336, pp. 14-25. 


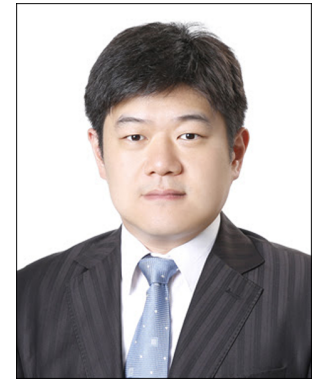

Taejin Choi is an assistant professor of sedimentary geology in the Department of Energy and Resources Engineering, Chosun University, Gwangju, Korea. He received his Ph.D. from School of Earth and Environmental Sciences, Seoul National University, Korea. He has studied provenance and thermal history of sedimentary basins in Korea using petrography, geochemistry, and geochronology of detrital minerals.

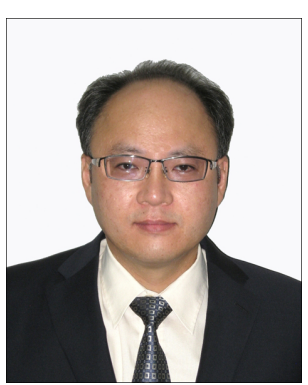

Hyoun Soo Lim is an associate professor of sedimentary geology in Department of Geological Sciences, Pusan National University, Busan, Korea. He received his Ph.D. from Seoul National University, Korea. His main research focuses on the low-temperature thermochronology, Quaternary geology, paleoclimate, geoarcheology and polar science. He won the Young Geologist Award (2008) and Academic Award (2019) from the Geological Society of Korea, and the Challenge Award (2009) from the Korea Polar Research Institute. He has served as an associate editor for the Journal of the Geological Society of Korea, and editorial board member for the Geosciences Journal.

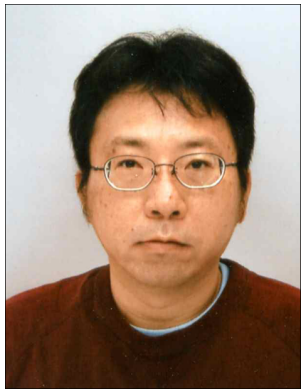

Yuji Orihashi is a professor of geology and geochemistry in the Department of Global Environment and Disaster Prevention Sciences, Graduate School of Science and Technology, Hirosaki University, Aomori, Japan. He received his DSc from Graduate School of Science, Hokkaido University, Japan. His scientific work focuses on U-Pb dating by LA-ICPMS and its development of the method. He has served as an editorial board member for Island Arc (until 2019) and as associate editor of Geochemical Journal. 